\title{
RECEIVED
}

\section{NOV 191998}

\section{Q.STI}

\section{Natural Gas Based Once-Through Fischer-Tropsch Design with Power Coproduction}

\author{
Topical Report \\ March 1998
}

Work Performed Under Contract No.: DE-AC22-91PC90027

\author{
For \\ U.S. Department of Energy \\ Office of Fossil Energy \\ Federal Energy Technology Center \\ P.O. Box 880 \\ Morgantown, West Virginia 26507-0880
}

By

Bechtel

Pittsburgh, Pennsylvania 15232 


\section{DISCLAIMER}

This report was prepared as an account of work sponsored by an agency of the United States Government. Neither the United States Government nor any agency Thereof, nor any of their employees, makes any warranty, express or implied, or assumes any legal liability or responsibility for the accuracy, completeness, or usefulness of any information, apparatus, product, or process disclosed, or represents that its use would not infringe privately owned rights. Reference herein to any specific commercial product, process, or service by trade name, trademark, manufacturer, or otherwise does not necessarily constitute or imply its endorsement, recommendation, or favoring by the United States Government or any agency thereof. The views and opinions of authors expressed herein do not necessarily state or reflect those of the United States Government or any agency thereof. 


\section{DISCLAIMER}

Portions of this document may be illegible in electronic image products. Images are produced from the best available original document. 


\section{TABLE OF CONTENTS}

\section{EXECUTIVE SUMMARY}

1 INTRODUCTION

2 OVERALL PLANT SUMMARY

3 OVERALL PLANT CONFIGURATION.

3.1 Simplified Process Flow Diagrams........................................... 3-4

3.2 Stream Flow Rates................................................................. 3-15

3.3 Utility Balance Summary.......................................................... 3-30

3.4 Overall Steam and Condensate Balance ......................................3-33

4 PROCESS DESCRIPTIONS - AREA 100, SYNGAS PREPARATION

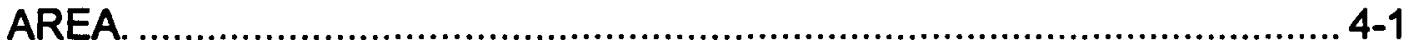

4.1 Plant 101 - Air Separation Plant.............................................. 4-2

4.2 Plant 102 - Autothermal Reforming Plant.......................................4-4

4.3 Plant $103-\mathrm{CO}_{2}$ Removal and Recycle Plant ................................. 4-6

5 PROCESS DESCRIPTIONS - AREA 200, FISCHER-TROPSCH SYNTHESIS AND PRODUCT UPGRADING AREA …........................5-1

5.1 Plant 201 - Fischer-Tropsch Synthesis Plant................................ 5-3

5.2 Plant 202 - Hydrogen Recovery Plant............................................ 5-7

5.3 Plant 203 - Hydrocarbon Recovery Plant...................................... 5-9

5.4 Plant 204 - Wax Hydrocracking Plant ........................................ 5-11

6 COMBINED-CYCLE POWER PLANT AND OFFSITES PLANTS.............. 6-1

6.1 Plant 31 - Combined-Cycle Power Plant........................................ 6-2

6.2 Offsites Plants........................................................................ 6 -4

7 OVERALL CATALYST AND CHEMICAL REQUIREMENTS …................. $7-1$

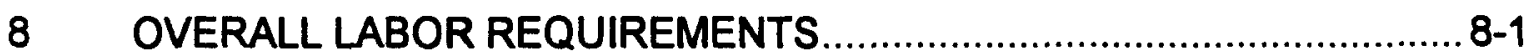

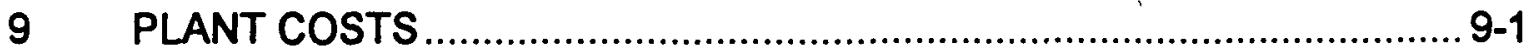

9.1 Installed Plant Costs .................................................................. 9-1

9.2 Operating Costs ...................................................................... $9-2$

10 SUMMARY AND RECOMMENDATIONS ............................................10-1

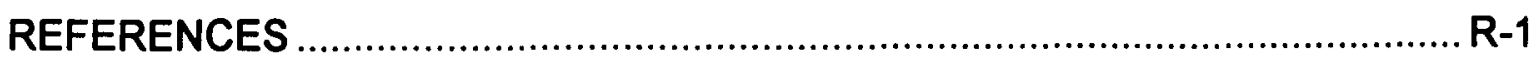

APPENDIX 


\section{EXECUTIVE SUMMARY}

A conceptual design for a once-through natural gas, Fischer-Tropsch (F-T) plant with power co-production has been developed to examine the economic viability of the conversion of natural gas to syncrude and/or liquid transportation fuels. This design incorporates an enriched air blown autothermal reformer for syngas generation and a proprietary catalytic process for F-T synthesis from Syncrude Technology, incorporated (STI). In developing this once-through F-T conceptual design, where applicable, individual plant designs and cost estimates were prorated directly from the F-T indirect coal liquefaction and natural gas studies. ${ }^{1,2}$

The plant processes $100 \mathrm{MMSCF} /$ day of natural gas and produces about $8815 \mathrm{BPD}$ of F-T liquid products. Primary liquid products are butanes, a C5-350 $\mathrm{F}$ naphtha and a $350-850^{\circ} \mathrm{F}$ distillate. The naphtha and distillate are raw products. Both are free of sulfur, nitrogen and oxygen containing compounds and can be further processed to produce premium gasoline and diesel blending stocks. The plant uses all of the byproduct steam and fuel gas production to supply its electric power requirements. In addition, it produces about $84.1 \mathrm{MW}$ of excess electric power. The only raw materials delivered to the plant are natural gas, water, catalysts and chemicals.

The following table gives a brief summary of the major feed and product streams to this once-through F-T liquefaction plant.

Once-Through, Natural Gas Fischer-Tropsch Liquefaction Plant Summary

\begin{tabular}{|c|c|c|c|}
\hline \multicolumn{4}{|l|}{ Feeds } \\
\hline $\begin{array}{l}\text { Natural Gas } \\
\text { Raw Water Make-un }\end{array}$ & $\begin{array}{r}100.0 \\
53\end{array}$ & MMSCF/da & (4,320 MMBtu/hr \\
\hline rimary Products & & & \\
\hline $\begin{array}{l}\text { F-T Naphtha } \\
\text { F-T Distillate } \\
\text { Butanes } \\
\text { Electric Dower }\end{array}$ & $\begin{array}{r}30.3 \\
64.6 \\
1.2 \\
2018.4\end{array}$ & $\begin{array}{l}\text { Mlbs/hr } \\
\text { Mlbs/hr } \\
\text { Mlbs/hr } \\
\text { MW-hr/dav }\end{array}$ & $\begin{array}{l}\text { (2,933 Bbl/day) } \\
\text { (5,736 Bbl/day) } \\
\text { (146 Bbl/day) } \\
\text { (84.1 MW) }\end{array}$ \\
\hline
\end{tabular}

Total capital cost for the plant is estimated at $415 \mathrm{MM} \$$. This is a mid-1996 cost for construction of the plant at a generic U. S. Gulf Coast location. The following table gives a brief summary of the estimated capital cost breakdown. 
Capital Cost Summary for the

Once-Through, Natural Gas Fischer-Tropsch Liquefaction Plant

\begin{tabular}{clcc} 
Area & Description & Cost (MM\$) & $\%$ ISBL \\
\cline { 2 - 3 } 200 & Syngas Preparation & 106.6 & 49.5 \\
& F-T Synthesis and & & \\
& $\quad$ Product Upgrading & 54.4 & 25.2 \\
31 & Power Generation & 54.5 & 25.3 \\
& Offsites Facilities & 120.3 & \\
& HO Service/Fees/Contingency & 79.4 & \\
& Total Cost: & 415.2 &
\end{tabular}

In the above table, Plant 31, the combined-cycle power generation plant, is considered an ISBL plant rather than an offsites plant as was done in the previous F-T design studies since the power generation facilities are a major cost component.

There are significant differences between the present once-through F-T design with that of the previous natural gas F-T study (Topical Report VI) the design of which is based on 1) recycling the unconverted syngas for maximum liquid production, and 2) producing fully upgraded gasoline and diesel blending stock as the main products. The once-through F-T power co-production design results in a lower overall plant capital cost. When compared at a same plant capacity of 100 MMSCFD of natural gas, this once through design costs about $32 \%$ less than that of the gas-recycle case. This reduction in capital cost is achieved at a slight sacrifice in the overall process thermal efficiency.

In addition to once-through versus gas recycling, there are other design differences between these two natural gas F-T studies. The once-through F-T design

1. Uses enriched air containing 40 mole \% oxygen for syngas generation compared to the previous gas-recycle design which uses 99.5 mole \% oxygen,.

2. Uses an autothermal reformer with $\mathrm{CO}_{2}$ recycle for syngas generation compared to the previous natural gas design which uses non-catalytic partial oxidation without $\mathrm{CO}_{2}$ recycle,

3. Uses STI's proprietary slurry-bed reactor F-T reactor design and catalyst information compared to the previous design which is based on the data of Satterfield et. al. (DOE contract DE-AC22-87PC79816)

4. Uses a simplified product recovery scheme which only produces raw naphtha and distillate products compared to the previous natural gas design which uses a more complex hydrocarbons recovery and product upgrading/ refining scheme to produce fully upgraded gasoline and diesel blending stocks, and

5. Contains a combined-cycle power plant based on a GE Frame 7 gas turbine for a state-of-the art power plant design . 
These differences are discussed in more detail in the report.

A preliminary economic analysis was carried out comparing this once-through F-T power co-production case with the previous maximum liquid production design using gas-recycle. This comparison, using the same methodology as in the previous studies, was made on the basis of

1. 100 MMSCFD plant of natural gas feed,

2. 1993 cost estimates,

3. 0.50 \$/MMBtu gas, and

4. 1996 EIA escalation factors.

In addition, the raw naphtha and distillate produced by the once-through design were treated as a combined high-value syncrude having a premium above that of conventional crude.

With a $0.50 \$ / M M B t u$ remote gas, the original F-T gas-recycle design had a COE (crude oil equivalent) price of $19.1 \mathrm{\$} /\left.\mathrm{bb|}\right|^{2}$. When this design is capacity-factored down from 412 MMSCFD to a 100 MMSCFD plant, the COE increased to about $23.0 \$ / b b l-$ approximately a $20 \%$ increase due solely to the loss in the economy of scale.

For the once-through, power co-production design, $84.1 \mathrm{MW}$ of excess power is produced. Assuming an electricity selling price of 3 cents per KWh to a IPP (independent power producer), this case has a COE of $20.6 \$ / \mathrm{bbl}$ with a syncrude premium factor of $10 \%$ (i.e., the combined F-T naphtha and distillate products have a value that is $10 \%$ higher than the price of petroleum crude due to its superior property). This represents a $12 \%$ decrease in the COE compared to the gas-recycle case.

The reduction in COE increases to $22 \%$ with an assumed syncrude premium factor of $20 \%$. It is not unreasonable to believe that F-T naphtha and distillate can command a selling price that is $20 \%$ higher than conventional petroleum crude since; in effect, the F-T product is equivalent to a bottomless, nitrogen-free and sulfur-free crude oil. Furthermore, under the right conditions, the benefit of F-T power co-production can be significant. This advantage could be what is needed to allow smaller once-through, Fischer-Tropsch gas liquefaction plants to be economically viable.

Site-specific studies are recommended to identify further economic improvements. For example, the following situations could result in additional capital cost savings.

1. Using associated gas or low-quality natural gas containing 10 to $12 \% \mathrm{CO}_{2}$

2. Using an air-blow, instead of an enriched air-blow, gasification process

3. Potable water production

4. Chemical co-production

5. Other site-specific symbiosis effects. 


\section{INTRODUCTION}

Bechtel, along with Amoco as the main subcontractor, developed a baseline design and a computer process simulation model for indirect coal liquefaction based on advanced Fischer-Tropsch (F-T) technology for the U. S. Department of Energy's (DOE's) Federal Energy Technology Center (FETC). This study was done under DOE Contract Number DE-AC22-91PC90027.

The objectives of the original 1991 contract were to develop:

- A Baseline design and two alternative designs for indirect liquefaction using advanced F-T technology. The Baseline design uses Illinois No. 6 coal and conventional upgrading and refining. One alternate case uses ZSM-5 upgrading of the vapor stream from the slurry F-T reactor. The other alternate case uses Western coal from the Powder River Basin.

- Capital and operating costs for the Baseline design and the alternatives. Individual plant costs for the alternative cases will be prorated on capacity, wherever possible, from the Baseline case.

- A process flowsheet simulation (PFS) model.

The baseline design, the economic analysis and PFS computer model will form a research planning tool with which PETC can use to plan, guide and evaluate its ongoing and future research and commercialization programs relating to indirect coal liquefaction for the manufacture of synthetic liquid transportation fuels.

The original study was divided into seven major tasks:

- Task 1:

Establish the Baseline design and alternatives.

- Task 2:

Evaluate the economics for the Baseline and alternate cases.

- Task 3: Develop engineering design criteria.

- Task 4: Develop a process flowsheet simulation (PFS) model for each case.

- Task 5: Perform sensitivity studies using the PFS model.

- Task 6: Document the PFS model and develop a.DOE training session on its use.

- Task 7: Perform project management, technical coordination and other miscellaneous support functions.

In 1995, the original study was extended to add the following four additional items

- Item 1: Improve the process flowsheet simulation model (PPS) for the alternative ZSM-5 upgrading case by adding additional 
components.

- Item 2: Develop another alternative ZSM-5 upgrading case using the

- Item 3: Western coal from the Powder River Basin as feedstock.

- Item 4: the F-T wax instead of hydrocracking.

Develop a case in which natural gas instead of coal is used as feedstock for Fischer-Tropsch indirect liquefaction.

In 1996, this study again was extended to investigate another natural gas, FischerTropsch liquefaction case. This case is for a smaller plant processing $100 \mathrm{MMSCF} /$ day of natural gas in a once-through mode with power co-production using the proprietary, Fischer-Tropsch, slurry-bed technology of Syncrude Technology, Incorporated. This present topical report covers the conceptual design of this once-through, FischerTropsch power co-production case.

\section{Design Scope:}

In the development of the previous coal and natural gas based F-T conceptual designs, both licensed (proprietary) and open art technologies were used. This philosophy was continued for this natural gas, once-through F-T study. With licensed technologies, reliance was made on data provided by the licensors and information to which Bechtel has access, but is not in the public domain. For open art technologies, Bechtel provided the process design.

The yields from and sizes of the Fischer-Tropsch slurry-bed synthesis reactors are based on the proprietary yield and kinetic data of STI (Syncrude Technology, Incorporated). The mechanical design of the F-T slurry-bed reactors is the same as that used in the previous studies, and reactor cost was obtained by capacity factoring. STI's proprietary "slurry valve" is included in the design for the catalyst/wax separation. The design of the mild hydrocracking plant, used for upgrading the F-T wax, is based on pilot plant data reported by Mobil, PARC, and UOP under DOE contract numbers DE-AC22-80PC30022, DE-AC22-89PC88400, and DE-AC22-85PC80017.

Following the philosophy of the previous F-T studies, the overall plant is designed to comply with all applicable environmental, safety and health regulations. Air cooling is maximized, whenever possible, in order to minimize cooling water requirements.

\section{Report Organization:}

This Topical Report contains ten major sections. Section 1 provides an introduction and general background of this DOE contract. Section 2 provides an overall material balance for the plant, capital cost estimates, a comparison between this once-through, F-T design with power co-production and the previous gas-recycle, maximum liquid production design. This section also contains the capital cost estimate for the construction of the plant at a generic U. S. Gulf Coast location. 
Section 3 of this report shows the overall configuration of this once-through F-T plant design and summarizes all the process flows for the entire complex. The facility is divided into two separate areas:

Area 100

Syngas Preparation

Area 200 F-T Synthesis and Product Upgrading

Figure 3.1 is a block flow diagram which shows the major process interconnections between the various plants. Section 3.1 contains simplified Process Flow Diagrams (PFDs) for all the individual ISBL plants. A simplified PFD also in included for Plant 31 , the combined-cycle power plant, since it is considered an ISBL plant because it is a major component of the total facility.

Section 3.2 contains the flow rates of the key streams of each process plant. These flow rates are presented in sequential order within the two major process areas by increasing stream number as shown in the PFDs. They are given either in mass (lb/hr) or mole (lb-mole/hr) bases. Component flow rates and conditions are given for each major stream entering and leaving a plant. However, some intermediate stream compositions and conditions within the F-T synthesis plant are not included to protect Syncrude Technology incorporated's proprietary technology.

Section 3.3 provides an utility balance summary for the entire complex. Section 3.4 contains a schematic representation of the overall steam and condensate balance for the complex.

Sections 4 and 5 contain detailed process descriptions for each ISBL plant within process Areas 100 and 200, respectively.

Section 6 describes the combined-cycle power plant and offsites plants. In this study, the combined-cycle power plant is considered and ISBL plant because of the importance of power production for this case.

Section 7 contains the overall plant catalyst and chemical requirements.

Section 8 contains the overall plant labor requirements.

Section 9 contains the cost information. In addition to the installed plant costs, this section contains the annual plant catalyst and chemical costs.

Section 10 summarizes this study and gives some recommendations for additional studies. 


\section{OVERALL PLANT SUMMARY}

Table 2.1 contains a summary of the major feed and product streams to this oncethrough, natural gas Fischer-Tropsch liquefaction plant. The plant processes 100 MMSCF/day of natural gas and produces about 8815 BPD of F-T liquid products. The primary liquid products are butanes, a $\mathrm{C} 5-350^{\circ} \mathrm{F}$ naphtha blending stock and a $350-850^{\circ} \mathrm{F}$ distillate. Both products are essentially free of sulfur, nitrogen and oxygen containing compounds. The naphtha and distillate products are not fully upgraded to gasoline and diesel blending components as were produced in the previous natural gas and coal-based F-T designs. ${ }^{1,2}$

Table 2.1

Once-Through, Natural Gas Fischer-Tropsch Liquefaction Plant Summary

Feed

Natural Gas

Raw Water Make-up

Primary Products

F-T Naphtha

F-T Distillate

Butanes

Electric power
100.0 MMSCF/day (4,320 MMBtu/hr)

5.3 MM Gal/day

$30.2 \mathrm{Mlbs} / \mathrm{hr}$

$64.6 \mathrm{Mlbs} / \mathrm{hr}$

$1.2 \mathrm{Mlbs} / \mathrm{hr}$

2018.4 MW-hr/day
(2,933 Bbl/day)

(5,736 Bbl/day)

(146 Bbl/day)

Table 2.2 shows the capital cost estimates for the plant. This is a mid-1996 cost for construction of the plant at a generic U. S. Gulf Coast location. There are significant differences between the present once-through F-T design with that of the previous natural gas F-T study (Topical Report VI) the design of which is based on 1) recycling the unconverted syngas for maximum liquid production, and 2) producing fully upgraded gasoline and diesel blending stock as the main products. The once-through F-T power co-production design results in a lower overall plant capital cost. Major design differences between the two cases are:

1. This case uses enriched air containing 40 mole $\%$ oxygen for syngas generation compared to the previous natural gas case which uses 99.5 mole \% oxygen.

2. This case uses an autothermal reformer with $\mathrm{CO}_{2}$ recycle for syngas generation compared to the previous natural gas case which uses noncatalytic partial oxidation.

3. This case is a once-through design compared to the previous natural gas case which contains a hydrogen recycle stream. 
4. This case only produces raw naphtha and distillate products compared to the previous case which produces fully upgraded gasoline and diesel blending stocks.

The reasons for and effects of these design differences are discussed in the report.

Table 2.2

Capital Cost Summary for the

Once-Through, Natural Gas Fischer-Tropsch Liquefaction Plant

$\begin{array}{clcc}\frac{\text { Area }}{100} & \text { Description } & \text { Cost (MM\$) } & \% \text { ISBL } \\ 200 & \text { Syngas Preparation } & 106.6 & 49.5 \\ & \text { F-T Synthesis and } & & \\ 31 & \text { Product Upgrading } & 54.4 & 25.2 \\ & \text { Power Generation } & 54.5 & 25.3 \\ & \text { Offsites Facilities } & 120.3 & \\ & \text { HO Service/Fees/Contingency } & 79.4 & \\ & \text { Total Cost: } & 415.2 & \end{array}$

The above plant costs are order-of-magnitude $+1-30 \%$ estimates.

In the above table, Plant 31, the combined-cycle power generation plant, is considered to be an ISBL plant, rather than an offsites plant as was the situation in the previous cases, since it is a major component of the total plant cost.

Section 9 contains more detailed information on the capital and operating costs for the plant. The economic implications of this plant design are discusses in a paper presented at the 1997 DOE 1997 Coal Liquefaction and Solid Fuels Contractors Review Conference. A copy of this paper is contained in the Appendix. 


\section{OVERALL PLANT CONFIGURATION}

This section presents an overall summary of this once-through, natural gas FischerTropsch indirect liquefaction plant with power co-production. It is divided into four subsections:

3.1 Simplified Process Flow Diagrams

3.2 Stream Flow Rates

3.3 Utility Balance Summary

3.4 Overall Steam and Condensate Balance

The plant is assumed to be located at a hypothetical U. S. Gulf Coast site.

Figure 3.1 is a block flow diagram for the two major process areas, Area 100 Synthesis Gas Preparation, and Area 200 - Fischer-Tropsch Synthesis and Product Upgrading. This diagram shows the major process interconnections between the primary ISBL plants in Areas 100 and 200. Plant 31, the combined-cycle power generation plant, is not shown on this diagram.

Area 100 contains three major plants:

- Plant 101, the Air Separation Plant

- Plant 102, the Autothermal Reforming Plant

- Plant 103, the $\mathrm{CO}_{2}$ Removal and Recycle Plant

Plant 101, the Air Separation Plant, includes air compression. The air separation section of Plant 101 produces a 95 mole \% oxygen stream which when blended with the compressed air from the air compressor produces an enriched air stream containing $\mathbf{4 0}$ mole \% oxygen that is sent to Plant 102 for synthesis gas generation.

Plant 102, the Autothermal Reforming Plant, is shown as three separate blocks in Figure 3.1. One block is the sulfur removal section which removes trace amounts of sulfur containing compounds from the natural gas by reaction with zinc oxide. Other blocks are used to indicate the autothermal reforming section and the heat recovery and syngas cooling section.

Plant 103, the $\mathrm{CO}_{2}$ Removal and Recycle Plant, is the final block shown in Area 100. This is a standard MDEA absorption plant which removes $\mathrm{CO}_{2}$ from a portion of the cooled syngas leaving Plant 102, and recycles it back to the autothermal reformer 


\section{Figure 3.1}

\section{Once-Through Fischer-Tropsch (F-T) Design With Power Coproduction}

(Overall Process Configuration)

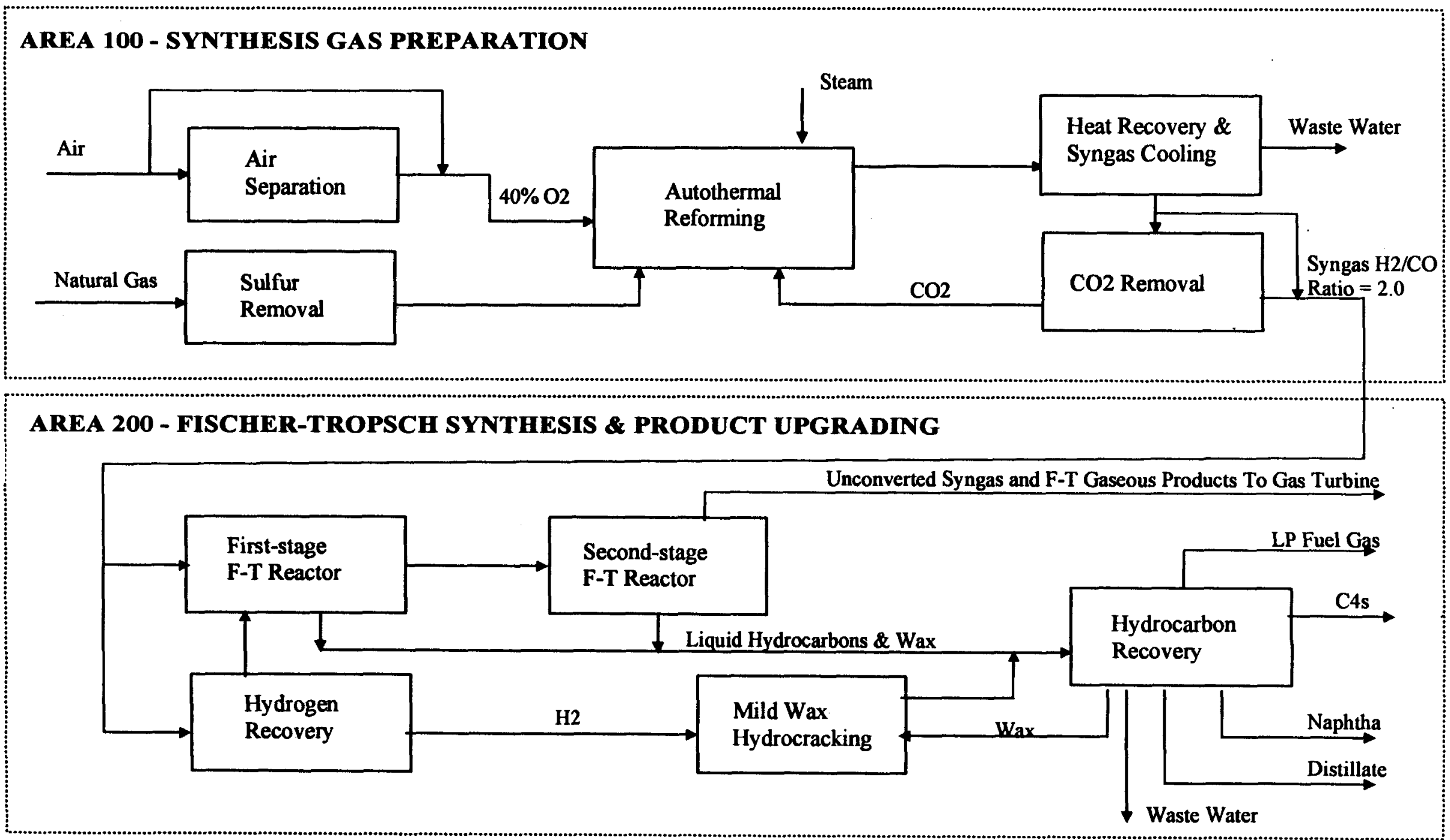


reactor section of Plant 102. The combined synthesis gas leaving Area 100 and going to Area 200 for Fischer-Tropsch synthesis is designed to have a molar $\mathrm{H}_{2} / \mathrm{CO}$ ratio of 2.01.

Area 200 contains four major plants:

- Plant 201, the Fischer-Tropsch Synthesis Plant

- Plant 202, the Hydrogen Recovery Plant

- Plant 203, the Hydrocarbon Recovery Plant

- Plant 204, the Wax Hydrocracking Plant

The combined synthesis gas leaving Area 100 is split into two streams. A small stream, about $1.4 \%$ of total flow, is split off from the main stream and sent to Plant 202 for hydrogen recovery. The reject syngas after hydrogen recovery is combined with the remaining syngas and sent to the first-stage F-T synthesis reactors in Plant 201. Plant 201, the Fischer-Tropsch Synthesis Plant is represented by two blocks in Figure 3.1; one block representing each F-T synthesis reactor stage. The unconverted syngas and light gases leaving Plant 201 goes to the gas turbine section of Plant 31, the Combined-Cycle Power Plant, which is not shown in Figure 3.1

The liquid hydrocarbons and wax produced in Plant 201 are sent to Plant 203, the Hydrocarbon Recovery Plant, where they are separated into a waste water stream and five product streams; a low pressure fuel gas stream, a butanes product stream, a naphtha product stream, a distillate product stream, and a wax stream. The wax stream is sent to Plan 204, the Wax Hydrocracking Plant, which cracks the wax to produce distillate, naphtha, butanes and low pressure fuel gas. 


\subsection{Simplified Process Flow Diagrams}

This section contains the simplified process flow diagrams (PFDs) for each process plant within both Areas 100 and 200 in PFDs 101-B-01 through 204-B-01. Each PFD is numbered according to the plant number for the plants in Process Areas 100 and 200. In addition, a simplified process flow diagram, PFD 31-B-01, is included for Plant 31, the combined-cycle power generation plant.

The offsite and utility plants are given Bechtel's conventional numbering code where 19 is Relief and Blowdown, 20 is Tankage, 21 is Interconnecting Piping, 30 is Electrical Distribution, 32 is Raw, Cooling and Potable Water Systems, etc. Equipment is numbered with the plant number followed by the Bechtel letter designation for that type of equipment followed by the sequential number designating the specific piece of equipment. If duplicates or spares are provided, these are given an additional letter designation in alphabetical order. For example, 201C-3A and 201C-3B are the two parallel first-stage, slurry-bed F-T reactors, and 201C-10 is the single F-T liquid/wax intermediate storage tank, all of which are in Plant 201.

In all of the above PFDs, major streams are designated by a number enclosed within a diamond. The component flow rates of these numbered streams are given in Tables $3.1,3.2$ and 3.3 in the following section.

Following the PFDs for the plants in process Areas 100 and 200, a simplified process flow diagram for Plant 31, the Combined-Cycle Power Generation Plant, is given in PFD 31-B-01. 


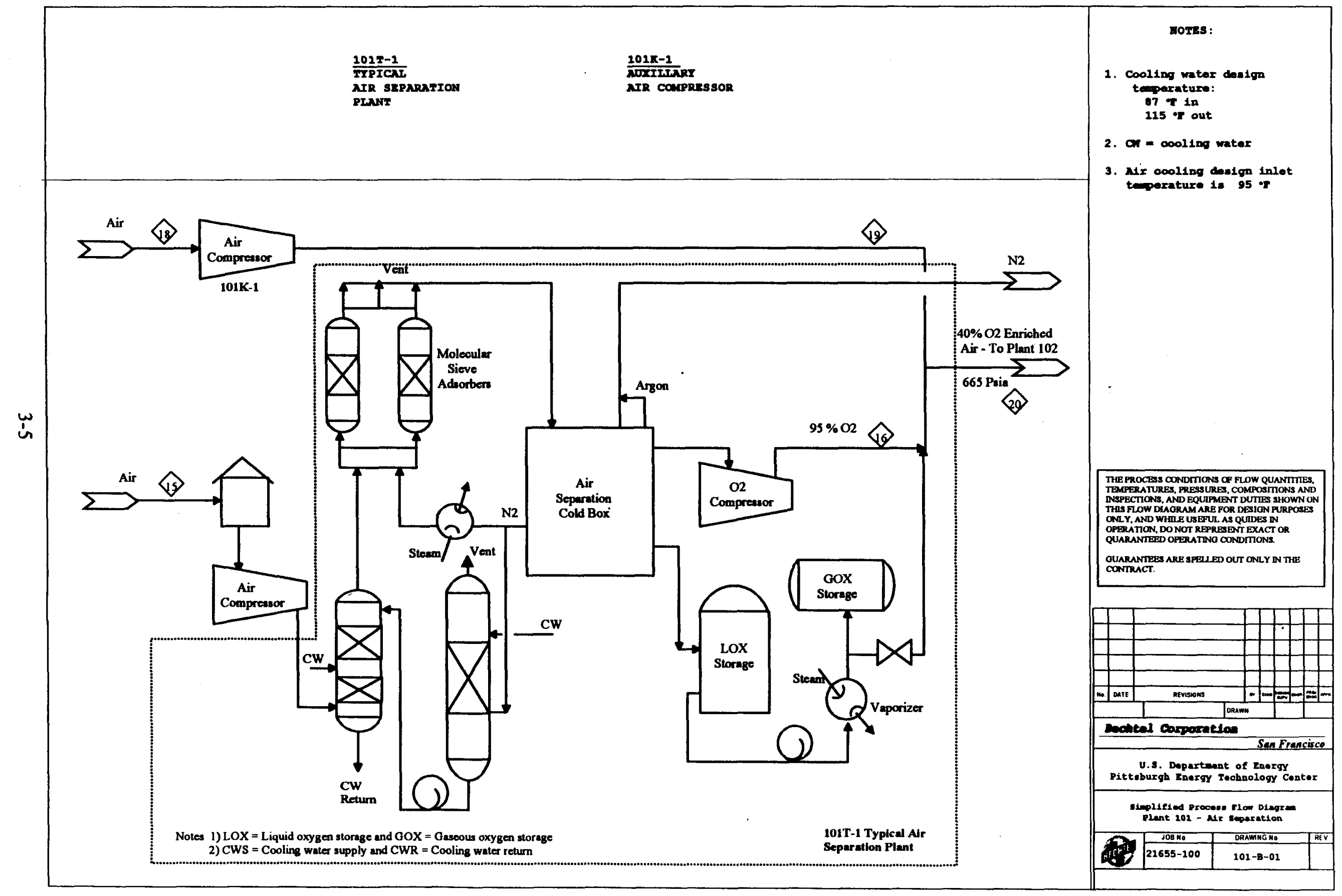

C: Sob216sostrntow Degramsi101 Bo1.054 


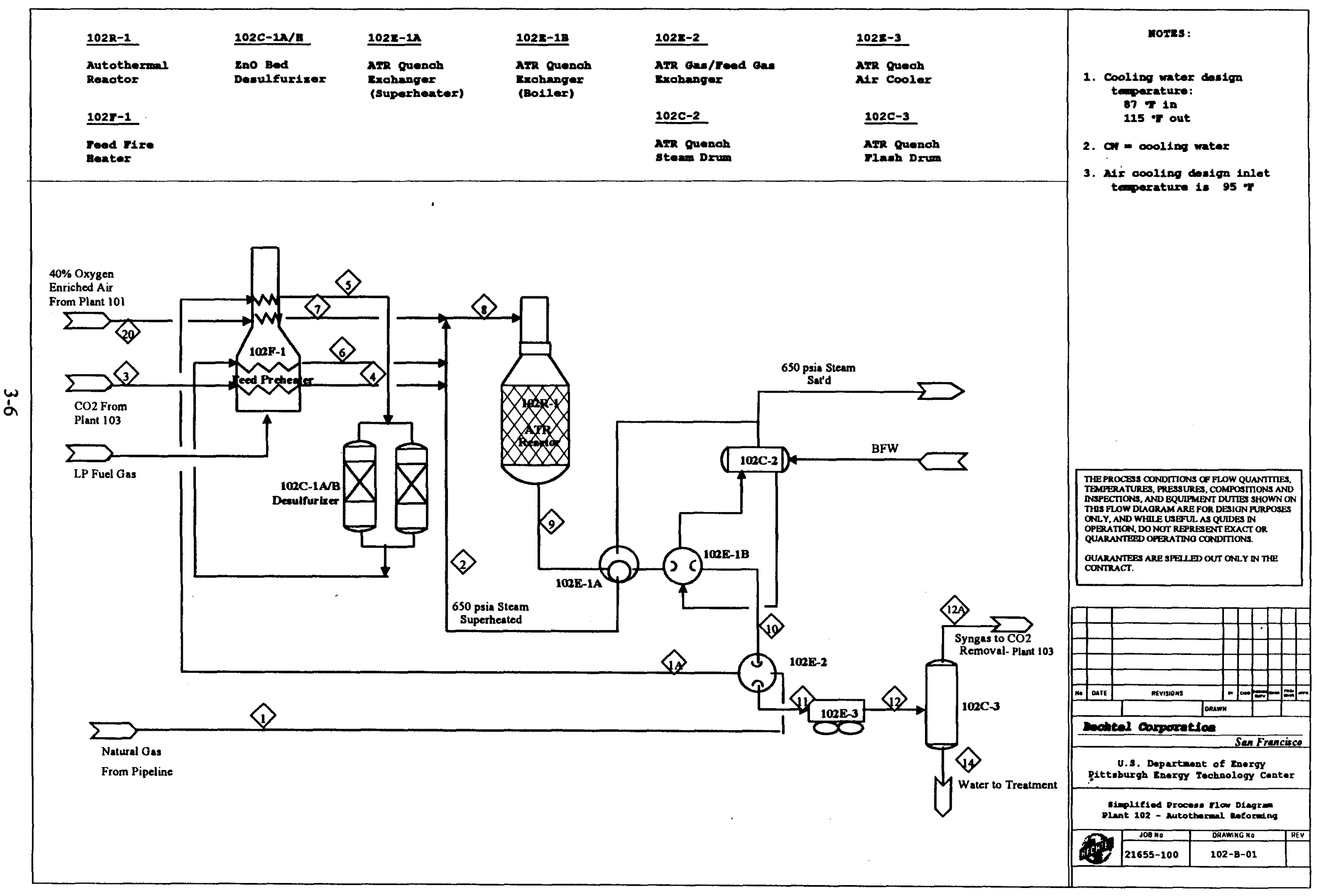

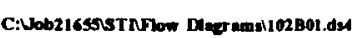




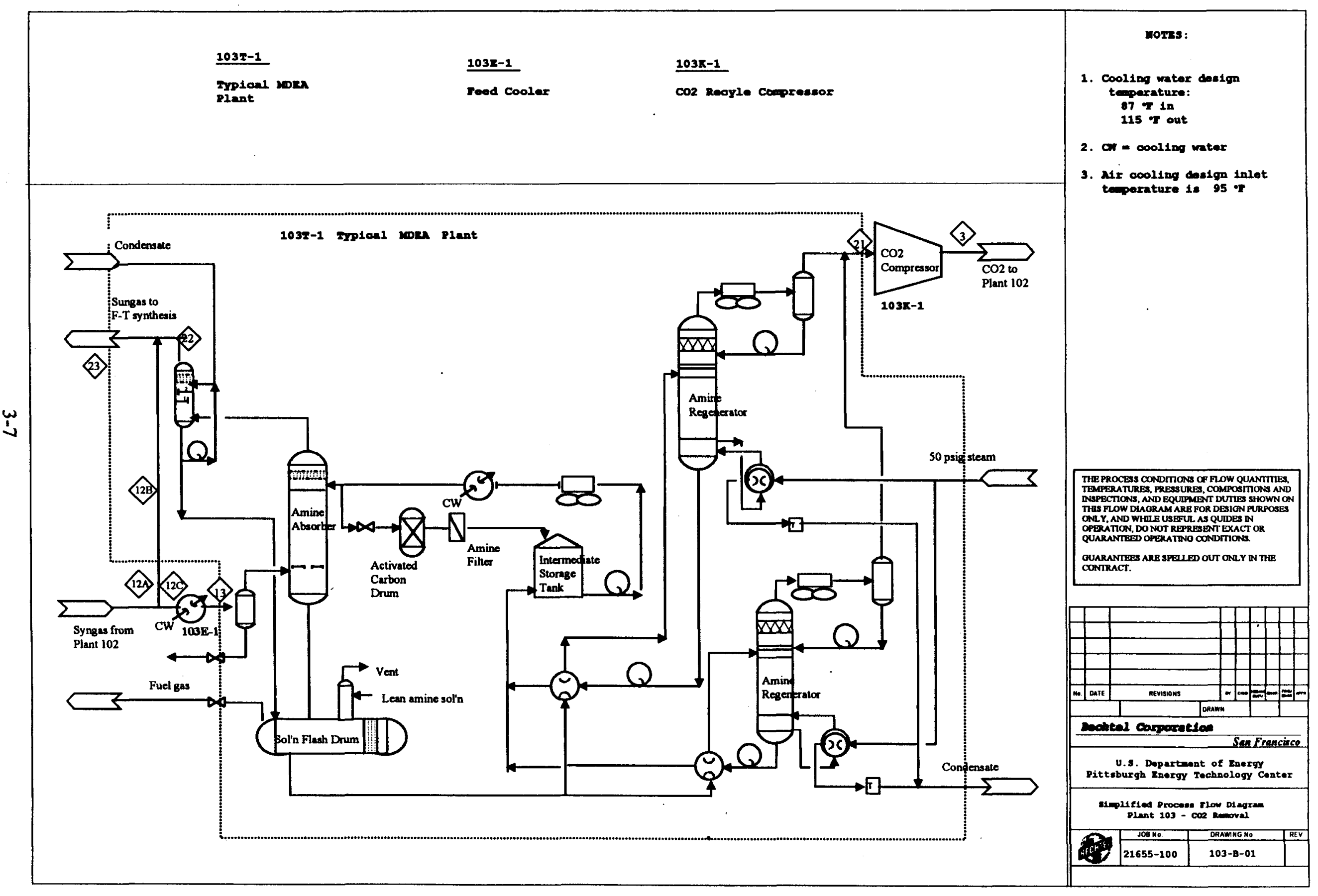

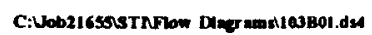




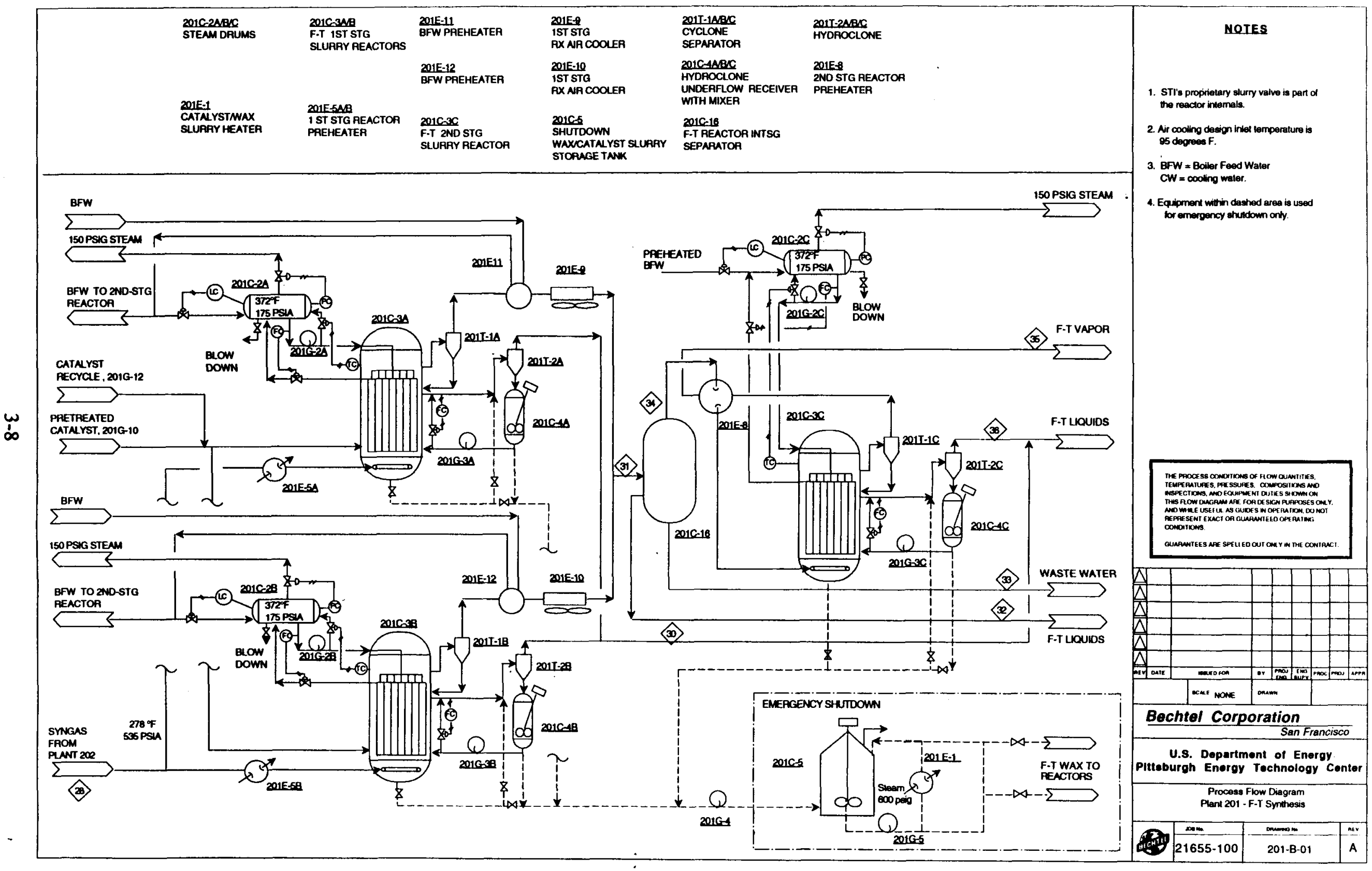




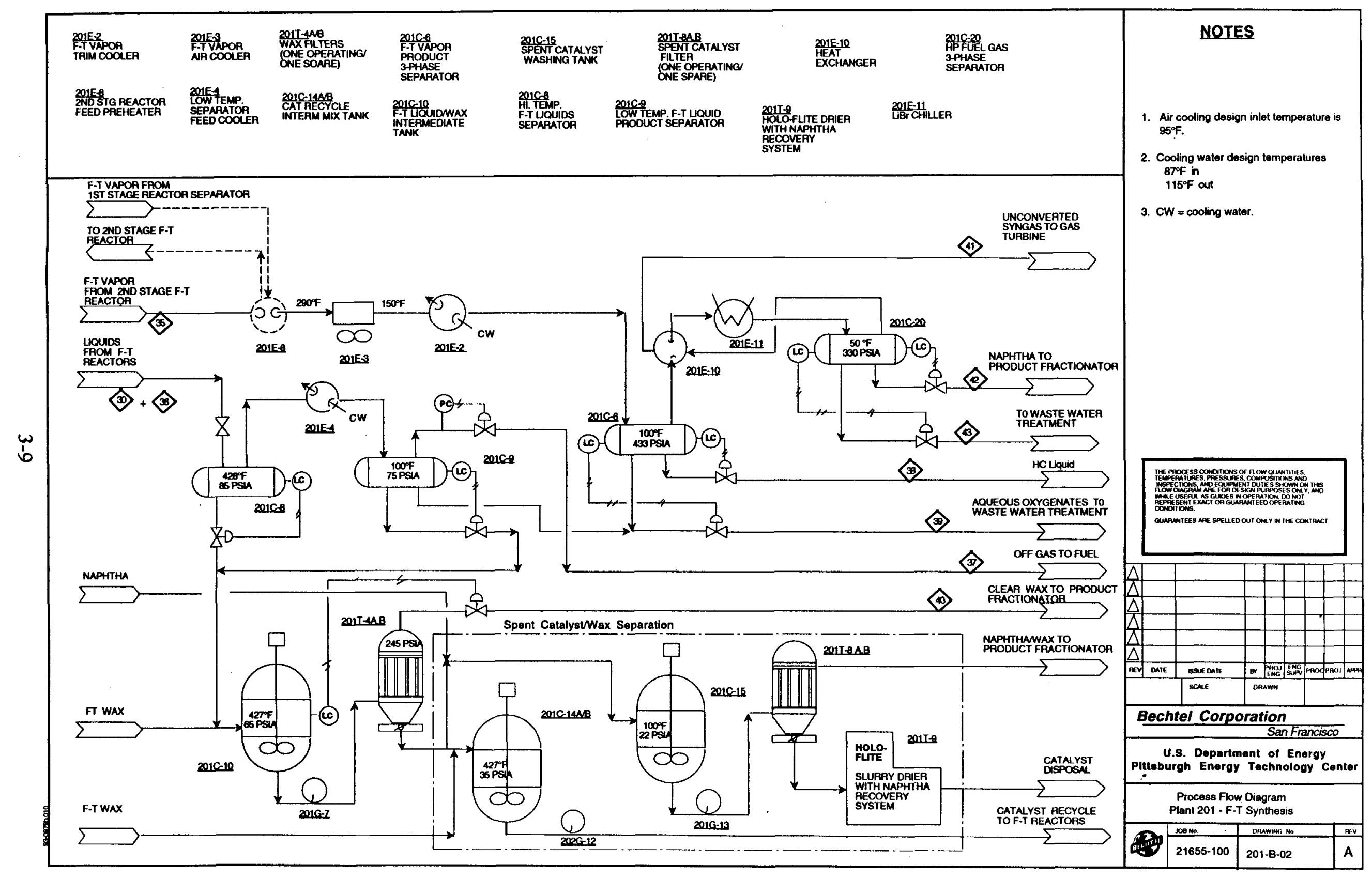




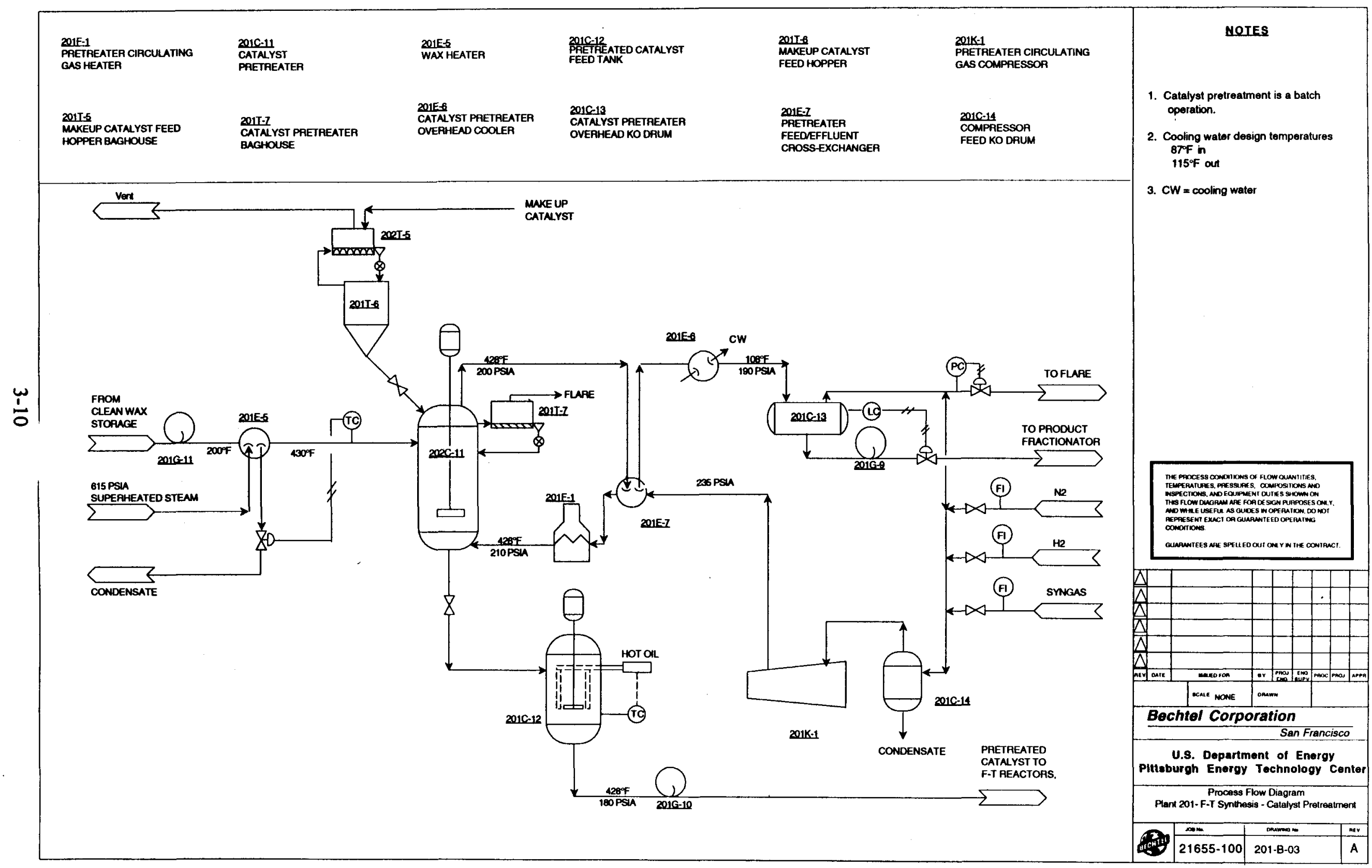




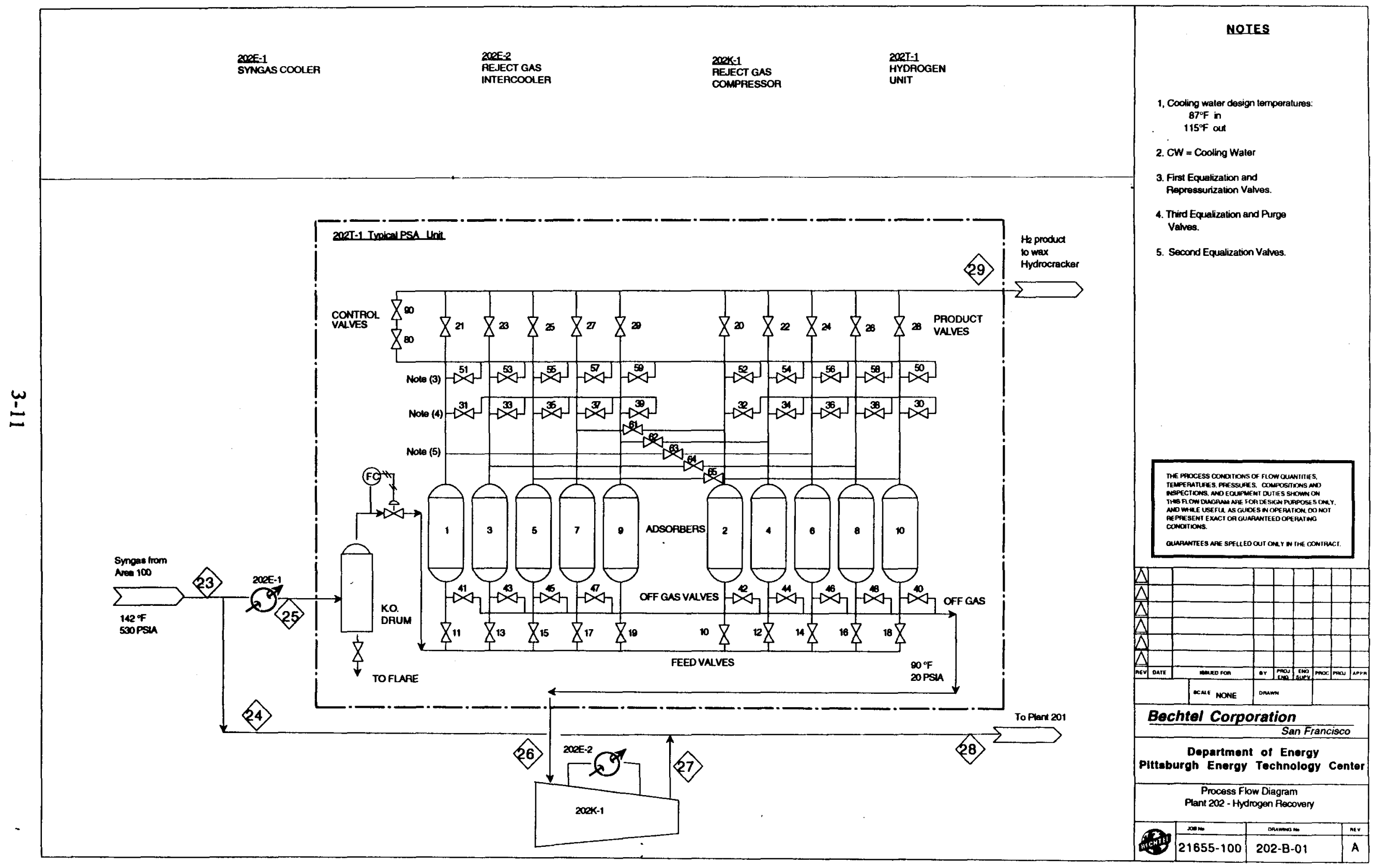




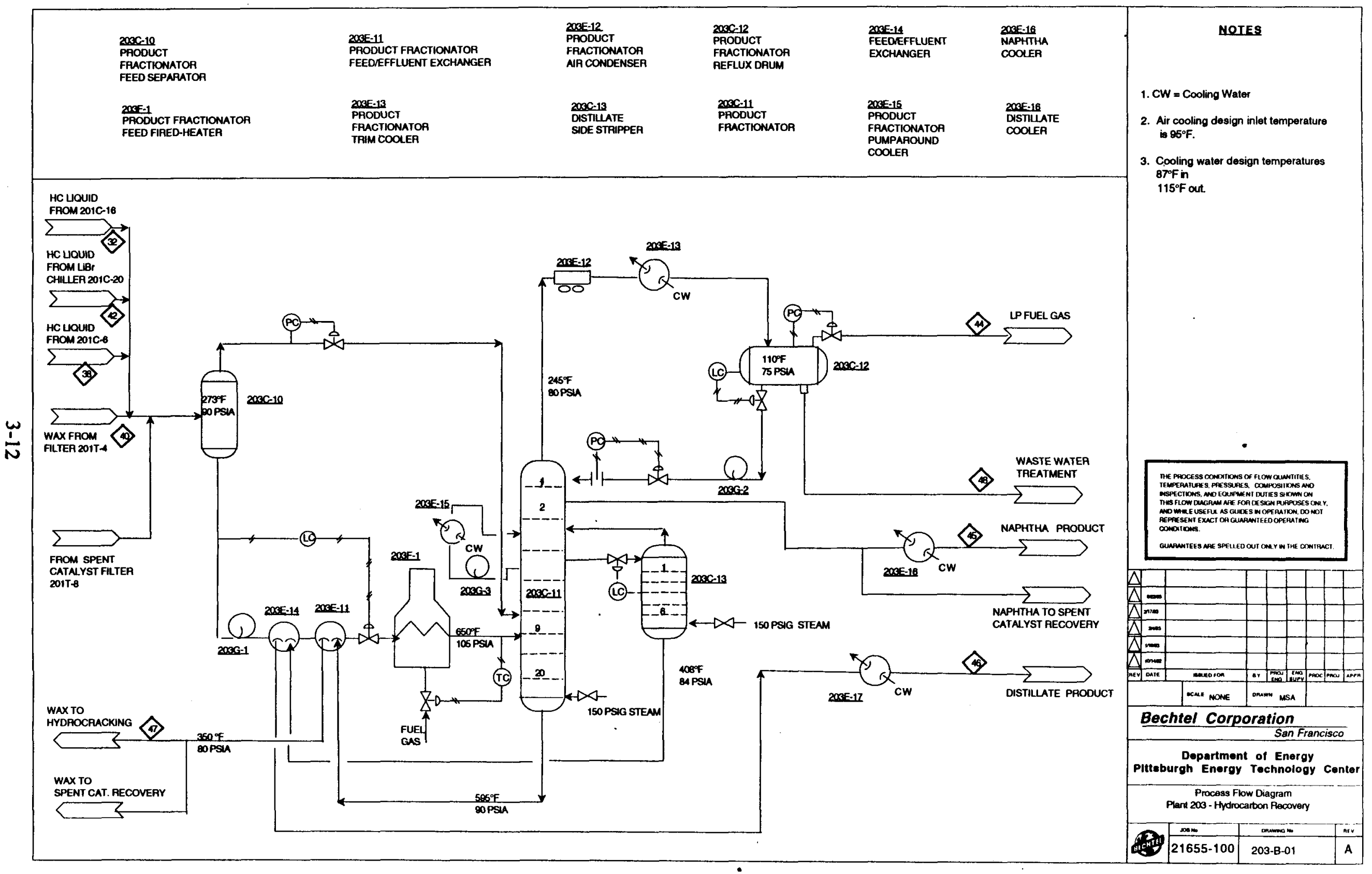




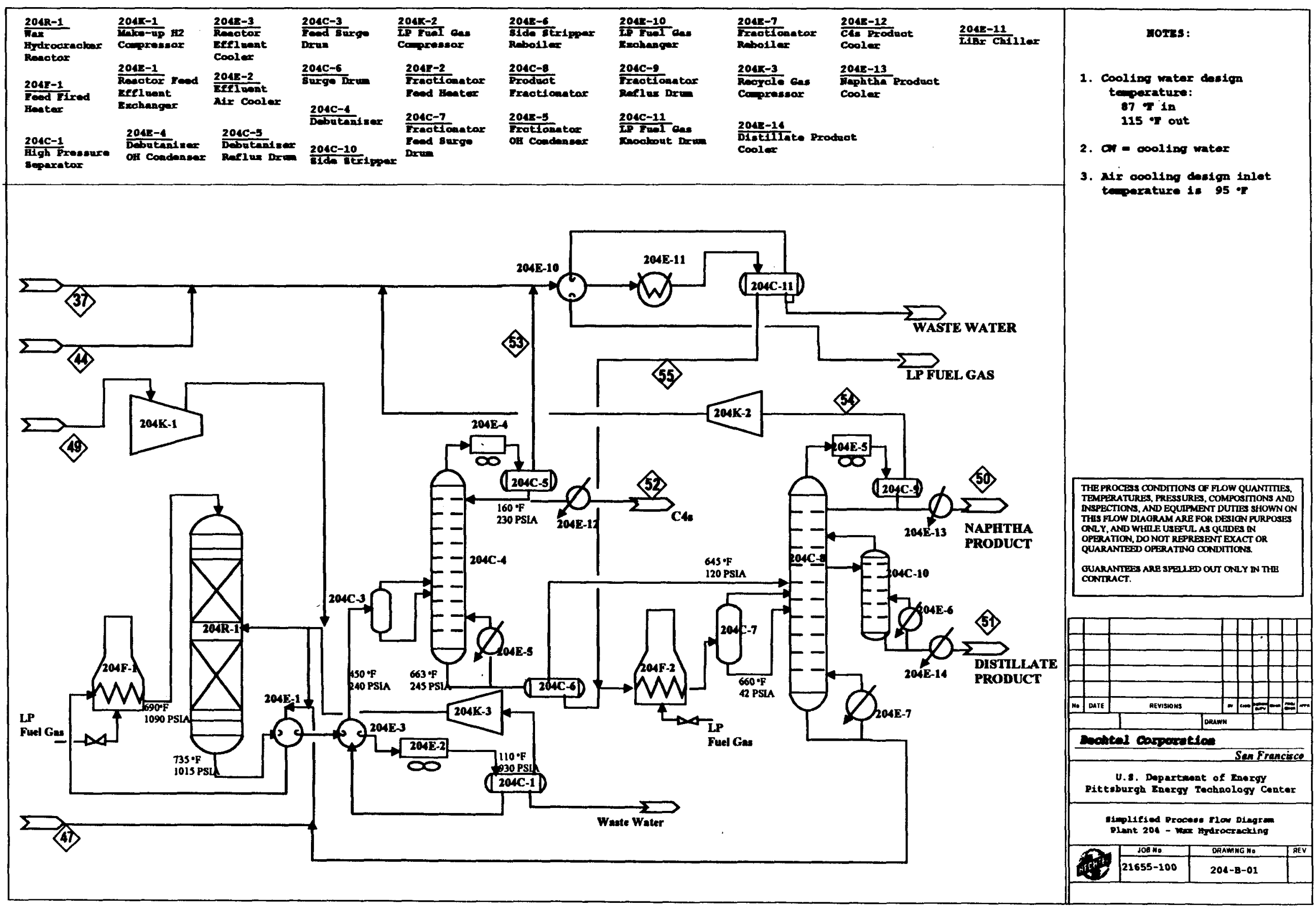

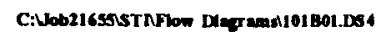




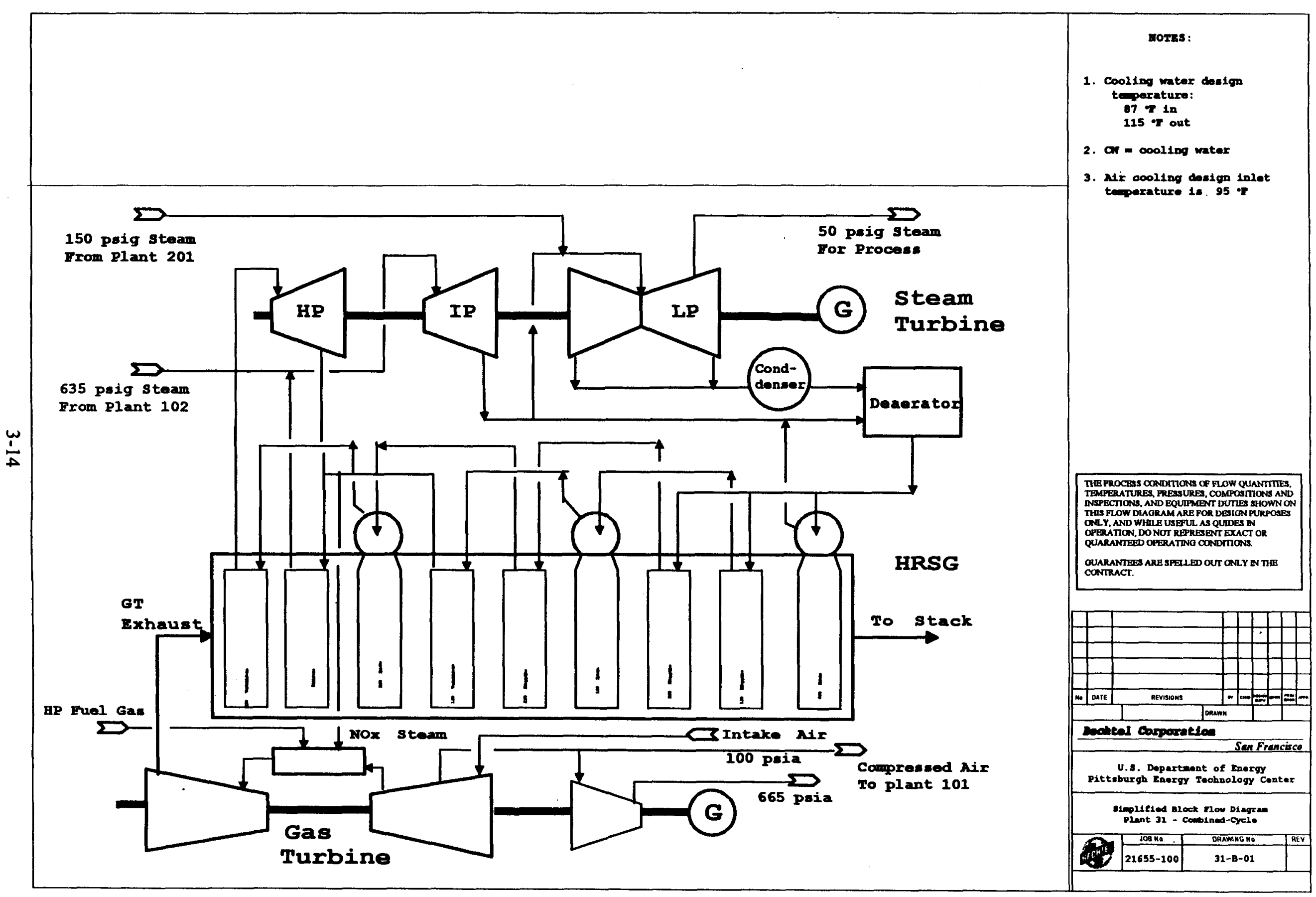




\subsection{Stream Flow Rates}

The component flow rates of key streams in process Areas 100 and 200 are shown in Tables 3.1, 3.2 and 3.3. The streams are identified by the same stream numbers used in the simplified PFDs shown in the previous section.

Table 3.1 contains the component molar flow rates, stream temperatures and pressures, total flow rates in both moles and weight, and the average stream molecular weights for the key streams in Area 100. Table 3.2 contains the same information for the process streams in Area 100 except that the component flow rates are given on a mass flow basis in $\mathrm{lbs} / \mathrm{hr}$.

Table 3.3 contains the component flow rates on a mass flow basis and stream conditions for the key process streams in Area 200. A few stream temperatures and pressures in Plant 201 have been omitted to protect the Fischer-Tropsch synthesis reactor conditions which Syncrude Technology, Incorporated considers to be proprietary. 
Table 3.1

Mole Flow, MPH

Syngas Preparation (Area 100) - Heat and Material Balances (1 of 4)

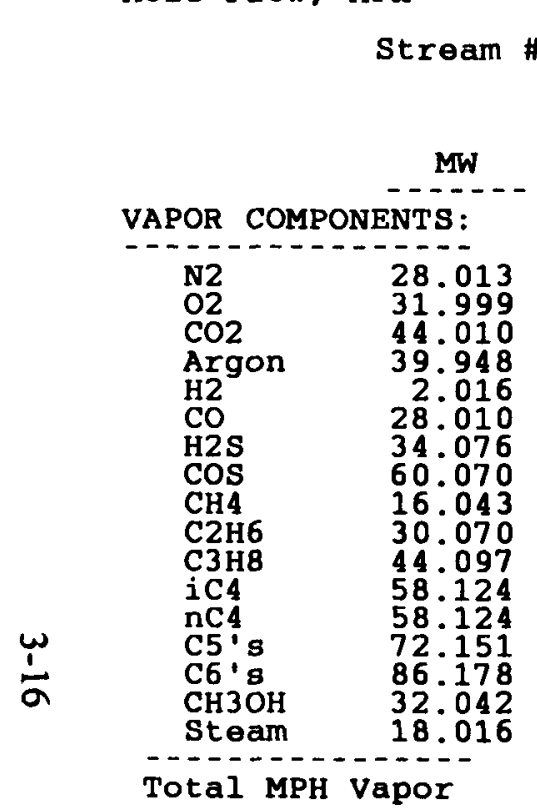

\begin{tabular}{|c|c|c|c|c|c|c|}
\hline $\begin{array}{c}1 \\
\text { Natural } \\
\text { Gas } \\
\text { Feod }\end{array}$ & $\begin{array}{c}1 A \\
\text { Natural Gas } \\
\text { Preheat for } \\
\text { ZnO Rx }\end{array}$ & $\begin{array}{c}2 \\
\text { Super Heat } \\
\text { Steam } \\
\text { Feed }\end{array}$ & $\begin{array}{c}3 \\
\text { Recyc1 co2 } \\
\text { after } \\
\text { Compression }\end{array}$ & $\begin{array}{l}4 \\
\text { Recycl } \mathrm{CO} 2 \\
\text { After Fired } \\
\text { Preheater }\end{array}$ & $\begin{array}{c}\text { NG to } \\
\text { Zno } \\
\text { Reactor }\end{array}$ & $\begin{array}{c}6 \\
\text { NG } \\
\text { to ATR }\end{array}$ \\
\hline$\cdots-\cdots$ & $\cdots-\cdots$ & $\cdots-\cdots$ & ------ & ------ & ------ & $-\cdots--$ \\
\hline $\begin{array}{r}63.7 \\
0.0 \\
78.0 \\
0.0 \\
0.0 \\
0.0 \\
0.0 \\
0.0 \\
10401.4 \\
347.0 \\
59.3 \\
8.8 \\
11.0 \\
11.0 \\
0.0 \\
0.0 \\
0.0\end{array}$ & $\begin{array}{r}63.7 \\
0.0 \\
78.0 \\
0.0 \\
0.0 \\
0.0 \\
0.0 \\
0.0 \\
10401.4 \\
347.0 \\
59.3 \\
8.8 \\
11.0 \\
11.0 \\
0.0 \\
0.0 \\
0.0\end{array}$ & $\begin{array}{r}0.0 \\
0.0 \\
0.0 \\
0.0 \\
0.0 \\
0.0 \\
0.0 \\
0.0 \\
0.0 \\
0.0 \\
0.0 \\
0.0 \\
0.0 \\
0.0 \\
0.0 \\
0.0 \\
8004.0\end{array}$ & $\begin{array}{r}0.0 \\
0.0 \\
2002.0 \\
0.0 \\
0.0 \\
0.0 \\
0.0 \\
0.0 \\
0.0 \\
0.0 \\
0.0 \\
0.0 \\
0.0 \\
0.0 \\
0.0 \\
0.0 \\
185.0\end{array}$ & $\begin{array}{r}0.0 \\
0.0 \\
2002.0 \\
0.0 \\
0.0 \\
0.0 \\
0.0 \\
0.0 \\
0.0 \\
0.0 \\
0.0 \\
0.0 \\
0.0 \\
0.0 \\
0.0 \\
0.0 \\
185.0\end{array}$ & $\begin{array}{r}63.7 \\
0.0 \\
78.0 \\
0.0 \\
0.0 \\
0.0 \\
0.0 \\
0.0 \\
10401.4 \\
347.0 \\
59.3 \\
8.8 \\
11.0 \\
11.0 \\
0.0 \\
0.0 \\
0.0\end{array}$ & $\begin{array}{r}63.7 \\
0.0 \\
78.0 \\
0.0 \\
0.0 \\
0.0 \\
0.0 \\
0.0 \\
10401.4 \\
347.0 \\
59.3 \\
8.8 \\
11.0 \\
11.0 \\
0.0 \\
0.0 \\
0.0\end{array}$ \\
\hline 10980.2 & 10980.2 & 8004.0 & 2187.0 & 2187.0 & 10980.2 & 10980.2 \\
\hline$-\frac{0.0}{0.0}$ & $\frac{0.0}{0.0}$ & $-\frac{0.0}{0.0}$ & $\begin{array}{r}0.0 \\
0.0\end{array}$ & $\begin{array}{r}0.0 \\
0.0\end{array}$ & $\begin{array}{c}0.0 \\
0.0\end{array}$ & $\begin{array}{c}0.0 \\
0.0\end{array}$ \\
\hline 10980.2 & 10980.2 & 8004.0 & 2187.0 & 2187.0 & 10980.2 & 10980.2 \\
\hline $\begin{array}{r}85.0 \\
730.0\end{array}$ & $\begin{array}{l}450.0 \\
695.0\end{array}$ & $\begin{array}{r}1000.0 \\
625.0\end{array}$ & $\begin{array}{l}312.9 \\
650.0\end{array}$ & $\begin{array}{r}1000.0 \\
625.0\end{array}$ & $\begin{array}{l}750.0 \\
670.0\end{array}$ & $\begin{array}{r}1000.0 \\
640.0\end{array}$ \\
\hline 187081 & 187081 & 144196 & 91441 & 91441 & 187081 & 187081 \\
\hline $\begin{array}{l}17.04 \\
17.04\end{array}$ & $\begin{array}{l}17.04 \\
17.04\end{array}$ & $\begin{array}{l}18.02 \\
18.02\end{array}$ & $\begin{array}{l}41.81 \\
41.81\end{array}$ & $\begin{array}{l}41.81 \\
41.81\end{array}$ & $\begin{array}{l}17.04 \\
17.04\end{array}$ & $\begin{array}{l}17.04 \\
17.04\end{array}$ \\
\hline
\end{tabular}

Notes: (1) Autothermal Reforming - Natural gas feed, 0.7 steam/carbon and

12) $0.5502 /$ carbon feed ratios. (2) 0 degF approach to both reforming and shift reactions, w/heat loss 
Table 3.1 (Continued)

Syngas preparation (Area 100) - Heat and Material Balances (2 of 4)

Mole Flow, MPH

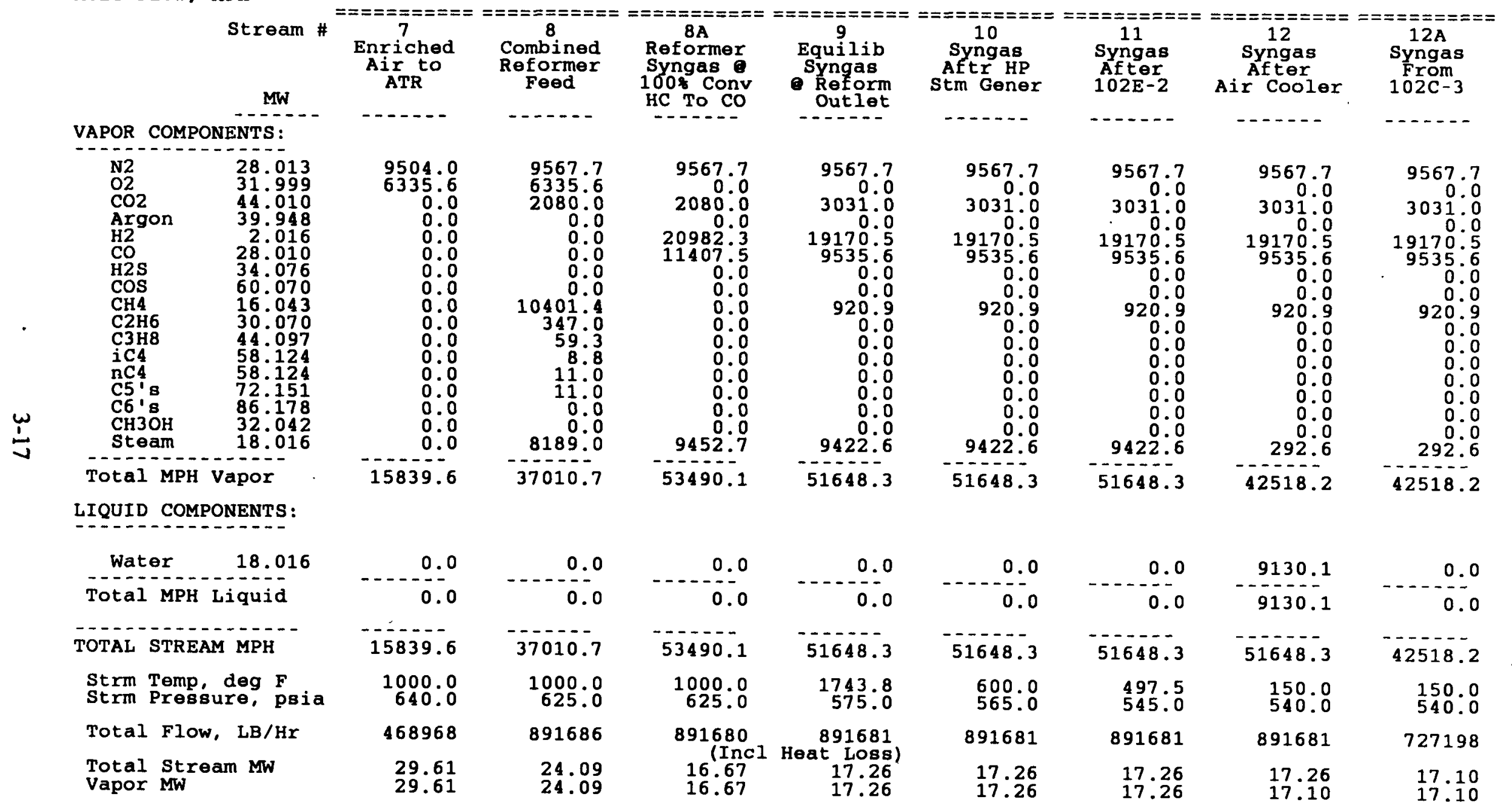


Table 3.1 (Continued)

Syngas Preparation (Area 100) - Heat and Material Balances (3 of 4)

Mole Flow, MPH

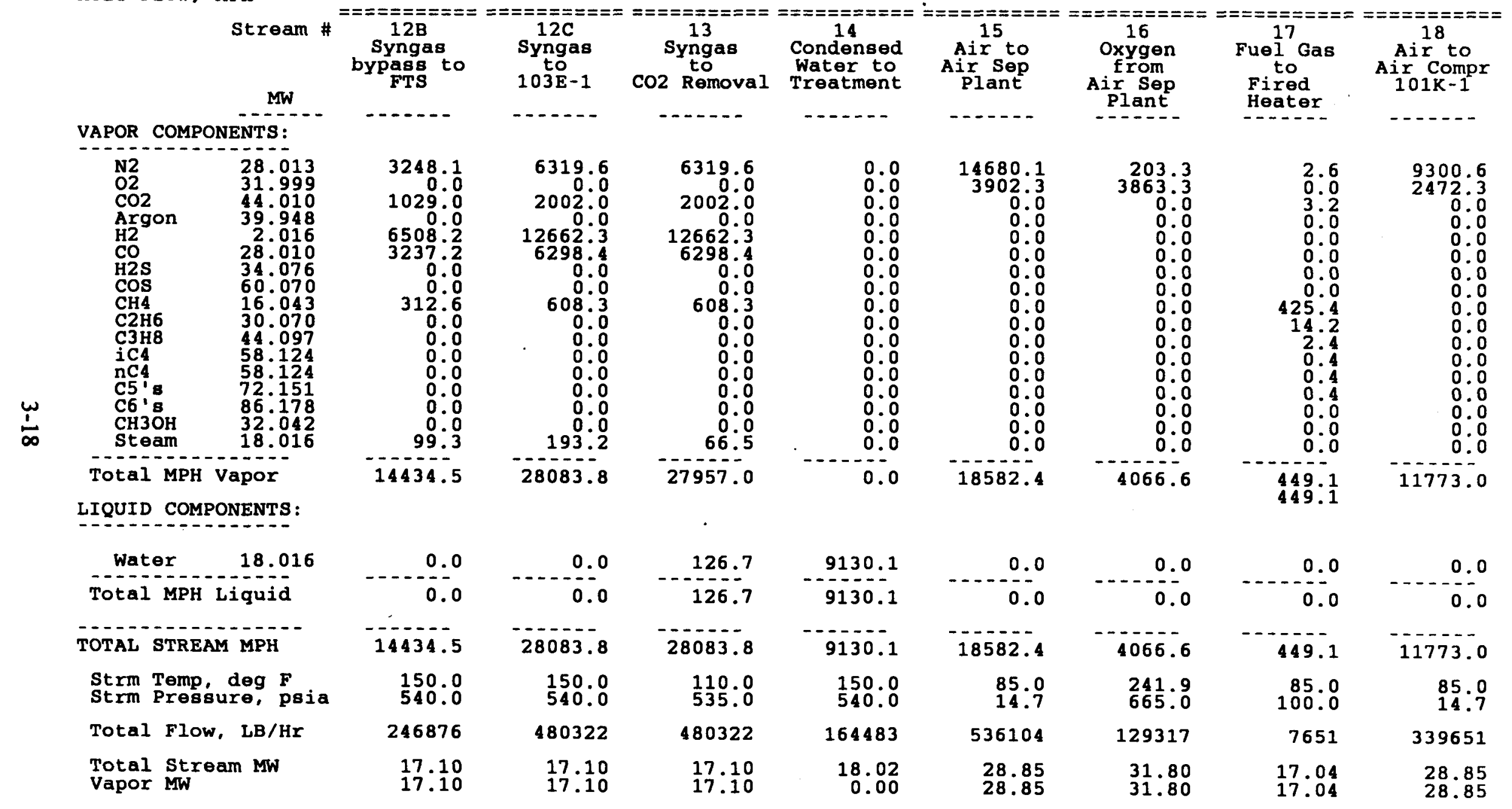


Table 3.1 (Continued)

Mole Flow, MPH

Syngas Preparation (Area 100) - Heat and Material Balances (4 of 4)

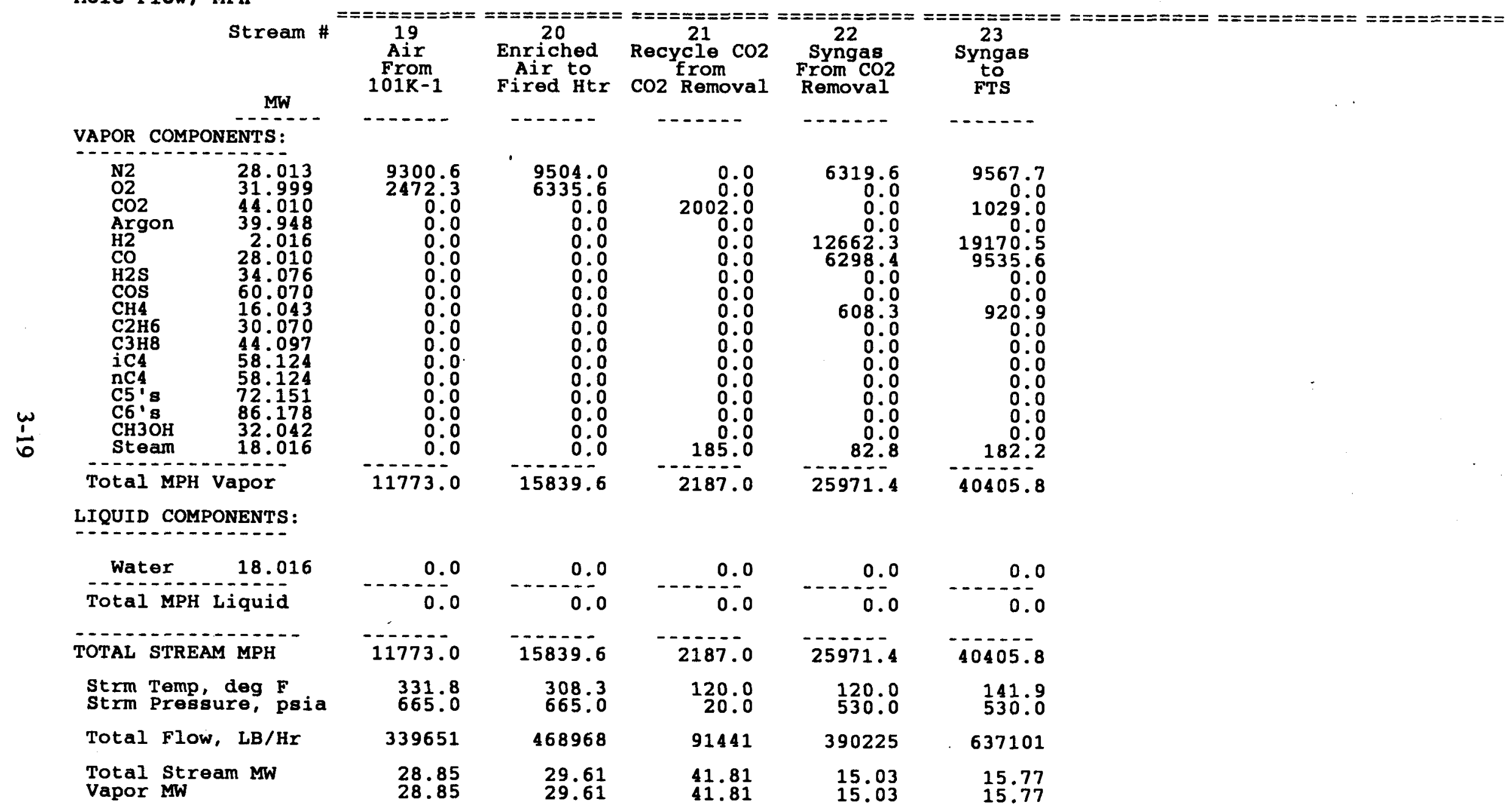


Table 3.2

Syngas Preparation (Area 100) - Heat and Material Balances (1 of 4)

Mass Flow, Lb/hr

\begin{tabular}{|c|c|c|c|c|c|c|c|}
\hline $\begin{array}{c}\text { Stream \# } \\
\text { MW }\end{array}$ & $\begin{array}{c}1 \\
\text { Natural } \\
\text { Gas } \\
\text { Feed }\end{array}$ & $\begin{array}{c}\text { 1A } \\
\text { Natural Gas } \\
\text { Preheat for } \\
\text { Zno Rx }\end{array}$ & $\begin{array}{l}2 \\
\text { Super Heat } \\
\text { Steam } \\
\text { Feed }\end{array}$ & $\begin{array}{l}\text { Recycl } \mathrm{CO} 2 \\
\text { after } \\
\text { Compression }\end{array}$ & $\begin{array}{l}\text { Recycl CO2 } \\
\text { After Fired } \\
\text { Preheater }\end{array}$ & $\begin{array}{c}\mathrm{NG}^{5} \text { to } \\
\text { Zno } \\
\text { Reactor }\end{array}$ & $\begin{array}{c}\text { Natural Gas } \\
\text { to ATR }\end{array}$ \\
\hline VAPOR COMPONENTS: & $\cdots-\cdots$ & ------ & $-\cdots--$ & ----- & $-\cdots--$ & $\cdots-\cdots$ & $--\cdots--$ \\
\hline $\begin{array}{lr}\text { N2 } & 28.013 \\
\text { O2 } & 31.999 \\
\text { CO2 } & 44.010 \\
\text { Argon } & 39.948 \\
\text { H2 } & 2.016 \\
\text { CO } & 28.010 \\
\text { H2S } & 34.076 \\
\text { COS } & 60.070 \\
\text { CH4 } & 16.043 \\
\text { C2H6 } & 30.070 \\
\text { C3H8 } & 44.097 \\
\text { iC4 } & 58.124 \\
\text { nC4 } & 58.124 \\
\text { C5's } & 72.151 \\
\text { C6's } & 86.178 \\
\text { CH3OH } & 32.042 \\
\text { Steam } & 18.016 \\
\end{array}$ & $\begin{array}{r}1784 \\
0 \\
3433 \\
0 \\
0 \\
0 \\
0 \\
0 \\
166870 \\
10434 \\
2615 \\
511 \\
639 \\
794 \\
0 \\
0 \\
0\end{array}$ & $\begin{array}{r}1784 \\
0 \\
3433 \\
0 \\
0 \\
0 \\
0 \\
0 \\
166870 \\
10434 \\
2615 \\
511 \\
639 \\
794 \\
0 \\
0 \\
0\end{array}$ & $\begin{array}{r}0 \\
0 \\
0 \\
0 \\
0 \\
0 \\
0 \\
0 \\
0 \\
0 \\
0 \\
0 \\
0 \\
0 \\
0 \\
0 \\
144196\end{array}$ & $\begin{array}{r}0 \\
0 \\
88108 \\
0 \\
0 \\
0 \\
0 \\
0 \\
0 \\
0 \\
0 \\
0 \\
0 \\
0 \\
0 \\
0 \\
3333\end{array}$ & $\begin{array}{r}0 \\
0 \\
88108 \\
0 \\
0 \\
0 \\
0 \\
0 \\
0 \\
0 \\
0 \\
0 \\
0 \\
0 \\
0 \\
0 \\
3333\end{array}$ & $\begin{array}{r}1784 \\
0 \\
3433 \\
0 \\
0 \\
0 \\
0 \\
0 \\
166870 \\
10434 \\
2615 \\
511 \\
639 \\
794 \\
0 \\
0 \\
0\end{array}$ & $\begin{array}{r}1784 \\
0 \\
3433 \\
0 \\
0 \\
0 \\
0 \\
0 \\
166870 \\
10434 \\
2615 \\
511 \\
639 \\
794 \\
0 \\
0 \\
0\end{array}$ \\
\hline Total LB/Hr Vapor & 187081 & 187081 & 144196 & 91441 & 91441 & 187081 & 187081 \\
\hline \multicolumn{8}{|l|}{ LIQUID COMPONENTS: } \\
\hline Water 18.016 & $\ldots$ & 0 & 0 & 0 & 0 & 0 & 0 \\
\hline Total LB/Hr Liquid & 0 & 0 & 0 & 0 & 0 & 0 & 0 \\
\hline TOTAL STREAM LB/HR & 187081 & 187081 & 144196 & 91441 & 91441 & 187081 & 187081 \\
\hline $\begin{array}{l}\text { Strm Temp, deg } \\
\text { Strm Pressure, psia }\end{array}$ & $\begin{array}{l}85.000 \\
730.00\end{array}$ & $\begin{array}{r}450.000 \\
695.00\end{array}$ & $\begin{array}{r}1000.000 \\
625.00\end{array}$ & $\begin{array}{r}312.857 \\
650.00\end{array}$ & $\begin{array}{r}1000.000 \\
625.00\end{array}$ & $\begin{array}{r}750.000 \\
670.00\end{array}$ & $\begin{array}{r}1000.000 \\
640.00\end{array}$ \\
\hline Total Flow, MPH & 10980.2 & 10980.2 & 8004.0 & 2187.0 & 2187.0 & 10980.2 & 10980.2 \\
\hline $\begin{array}{l}\text { Total Stream MW } \\
\text { Vapor } \mathbf{M W}\end{array}$ & $\begin{array}{l}17.04 \\
17.04\end{array}$ & $\begin{array}{l}17.04 \\
17.04\end{array}$ & $\begin{array}{l}18.02 \\
18.02\end{array}$ & $\begin{array}{l}41.81 \\
41.81\end{array}$ & $\begin{array}{l}41.81 \\
41.81\end{array}$ & $\begin{array}{l}17.04 \\
17.04\end{array}$ & $\begin{array}{l}17.04 \\
17.04\end{array}$ \\
\hline
\end{tabular}

Notes: (1) Autothermal Reforming - Natural gas feed, 0.7 steam/carbon and

(2) 0 degF approach to both reforming and shift reactions, w/heat $108 s^{\circ}$. 
Table 3.2 (Continued)

Syngas Preparation (Area 100) - Heat and Material Balances (2 of 4)

Mass Flow, Lb/hr

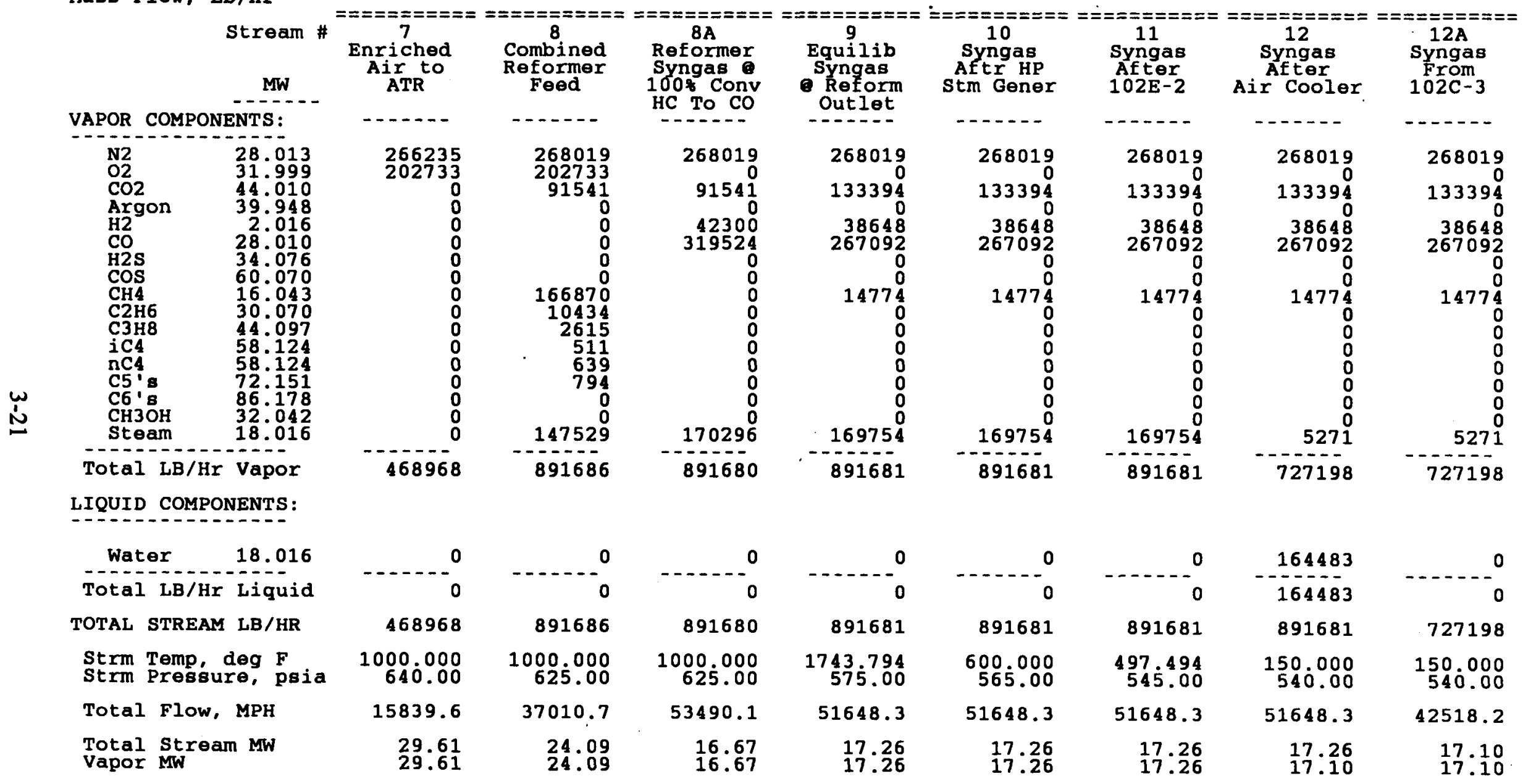


Table 3.2 (Continued)

Syngas Preparation (Area 100) - Heat and Material Balances (3 of 4)

Mass Flow, Lb/hr

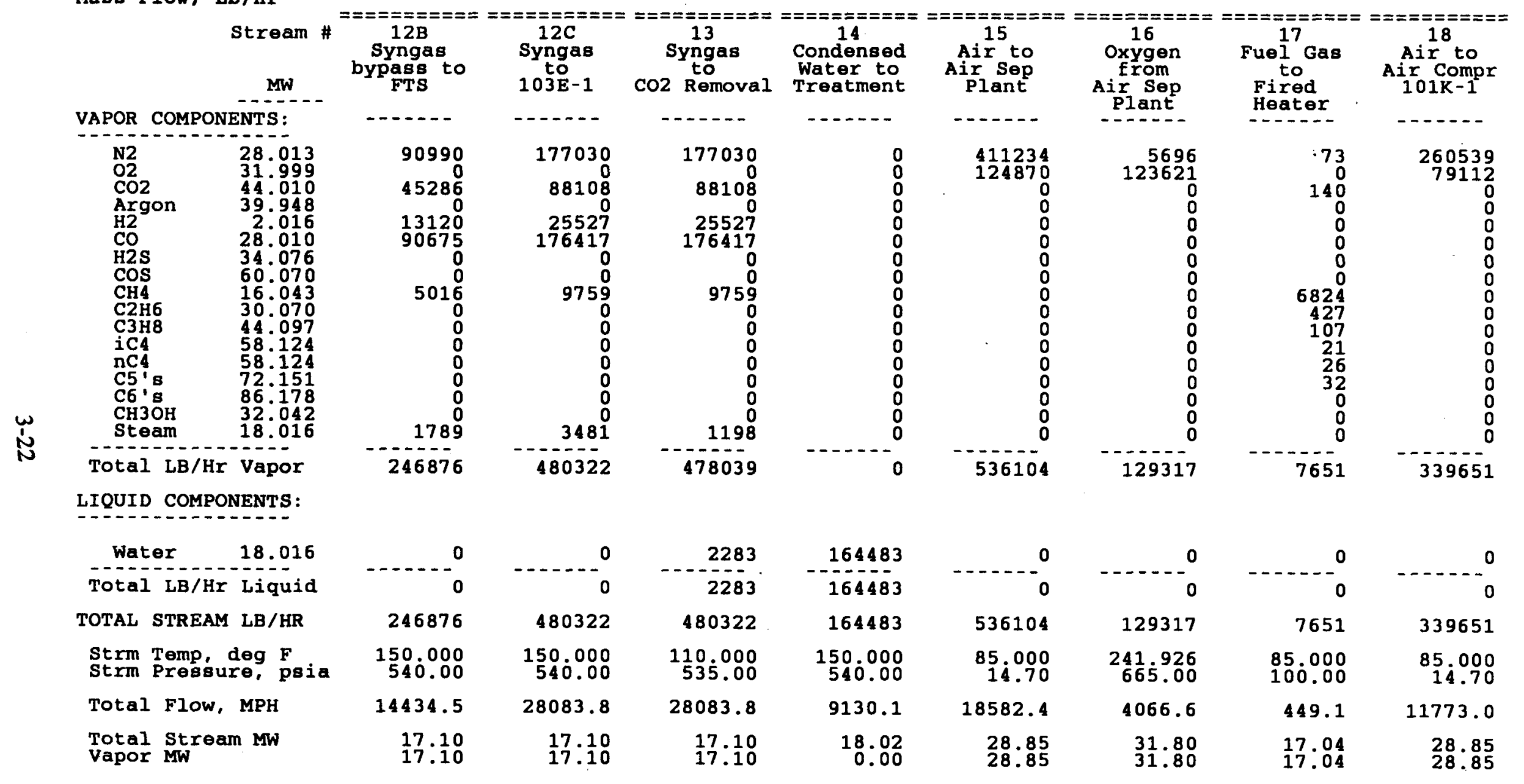


Table 3.2 (Continued)

Syngas Preparation (Area 100) - Heat and Material Balances (4 of 4)

Mass Flow, Lb/hr

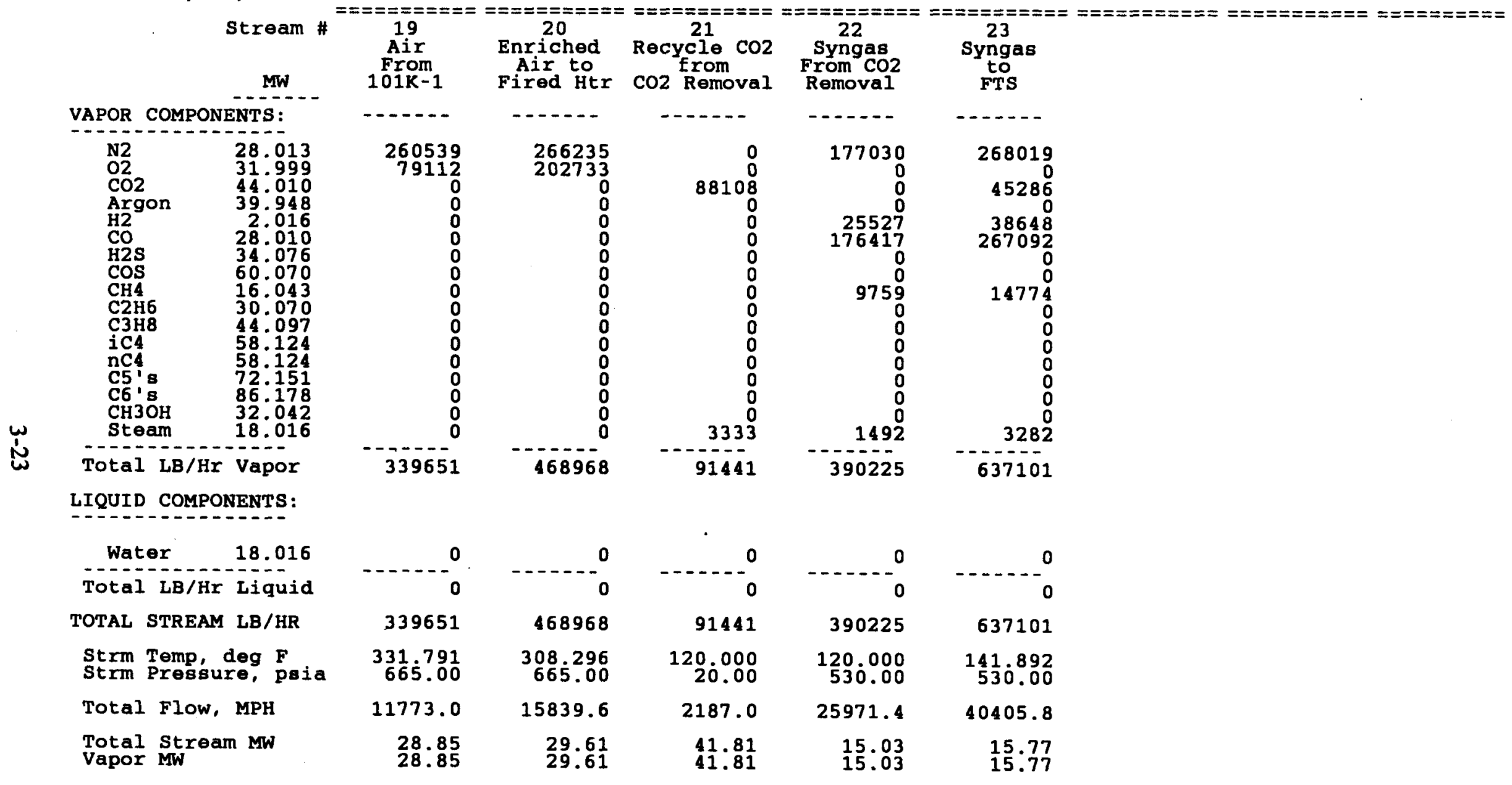




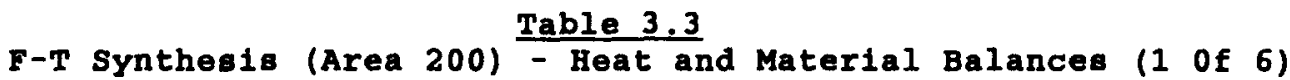

Mass Flow, Lb/hr

Stream \#

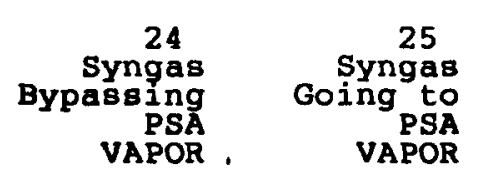

Components:

$\mathrm{H} 2$

N2

$\mathrm{CO} 2$

$\mathrm{H} 2 \mathrm{O}$

$\mathrm{CH} 4$

C2H4

C3H6

IC4 8

NC4 8

IC4 H10

w NC4H10

i $\quad c 5 s$

C6-C10

C11-C19

C7 $-300 \mathrm{HC}$

$300-350 \mathrm{HC}$

$500+\mathrm{HC}$

Oxygenates

Total Flow LB/HR

Total Flow LBMOL/HR

Temperature deg F
Pressure Paia
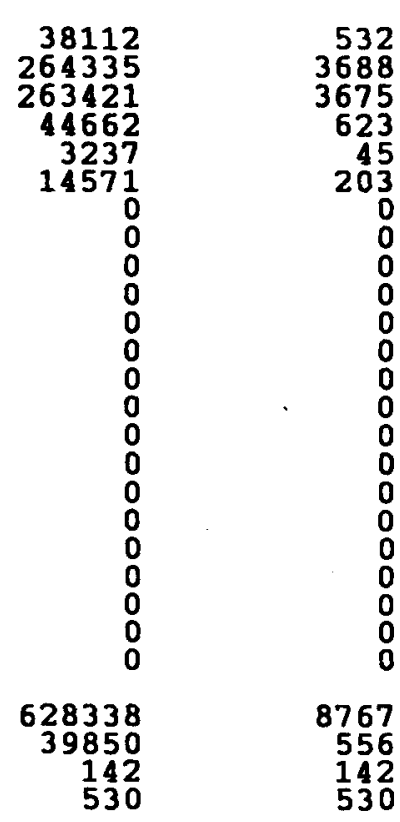

Compressed pressed
Reject
Gas
VAPOR 28
Feed To
1st-stg FT
Reactors
VAPOR 148
3635
3675
623
34
203
0
0
0
0
0
0
0
0
0
0
0
0
0
0
0
0
0
8319
363
278
530 $\begin{array}{rr}29 & 30 \\ \text { H2 } & \text { To } \\ \text { Wax } & \text { Liquids } \\ \text { HC } & \text { Reactors) } \\ \text { VAPOR } & \text { LIQUID }\end{array}$

148
3635
3675
623
45
203
0
0
0
0
0
0
0
0
0
0
0
0
0
0
0
0
0
8330
364
90
20

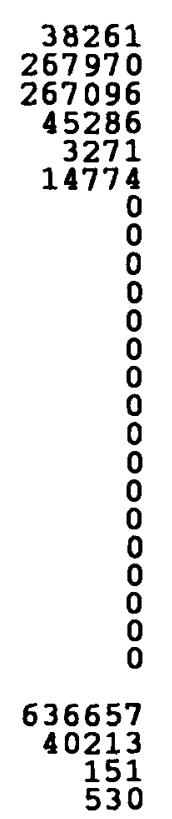

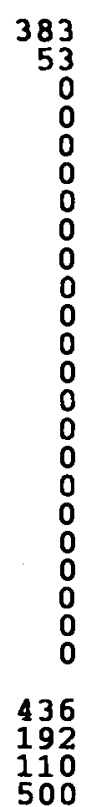

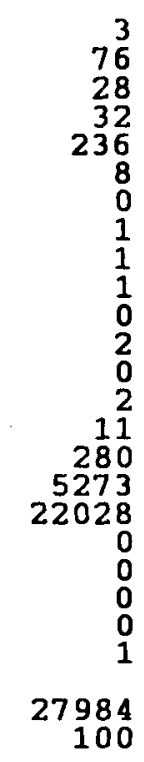


F-T Synthesis (Area 200) - Heat and Material Balances (2 0 f 6)

Mass Flow, Lb/hr

stroam \#

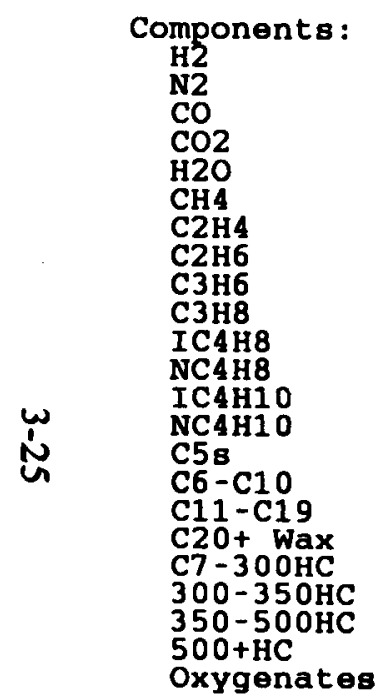

Total Flow LB/HR

Total Flow LBMOL/HR

Temperature deg
Pressure Psia
FT Vapors (1st-stg Reactors)

FT Liquids Interstage Ro Drum)

33 Waste Water LIOUID

34
Unconv. Gas
(Interstage
KO Drum)

Unconv 35 Fir (2nd-stg

FT Liquids $\begin{array}{r}36 \\ \text { (2nd-stg }\end{array}$ FT Reactor

LIQUID Off 37 To Fuel

VAPOR
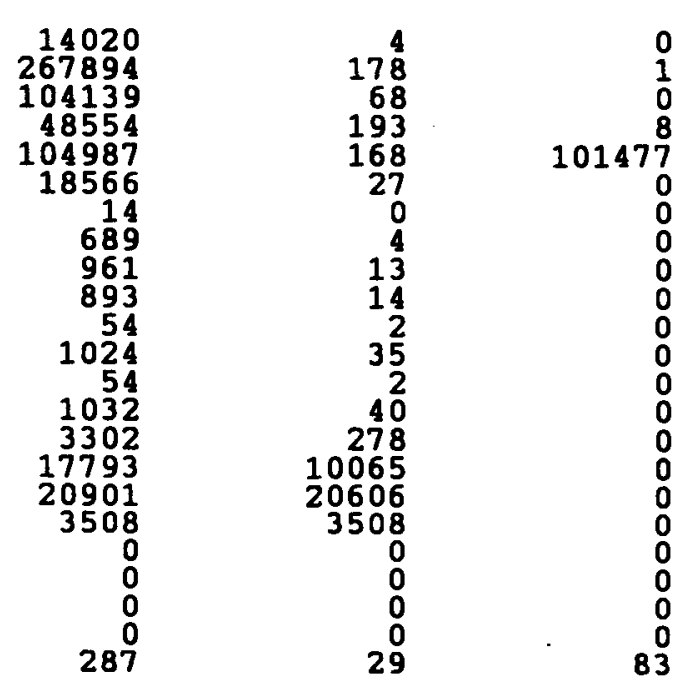

14016

267715

267715

VAPOR

$3342 \quad 43029$

$18539 \quad 2001$

14

685

948

879

989

53
992

992
3024

294

0

0
0
0

0
175

101570

608672
28762

233
150

150
484

9า

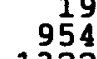

1322

1227

1388

74
1394

4310

14690

9422
2454

471868

22893
150

150
484

463122
18443

3

30
37

2
10

(a) 
Table 3.3 (Continued)

F-T Synthesis (Area 200) - Heat and Material Balances (3 of 6)

Mass Flow, Lb/hr

\begin{tabular}{|c|c|c|c|c|c|c|c|c|}
\hline stream \# & $\begin{array}{c}38 \\
\text { HC Liquids } \\
\text { (Low Temp } \\
\text { Separator) } \\
\text { LIQUID }\end{array}$ & $\begin{array}{r}39 \\
\text { W. Water } \\
\text { With } \\
\text { Oxygenates } \\
\text { LIQUID }\end{array}$ & $\begin{array}{r}40 \\
\text { Wax To } \\
\text { Product } \\
\text { ractionator } \\
\text { LIQUID }\end{array}$ & $\underset{\text { Gas }}{\text { HP }}$ & $\begin{array}{l}41 \\
\text { Unconv. } \\
\text { Gas To } \\
\text { Turbine } \\
\text { VAPOR }\end{array}$ & $\begin{array}{r}42 \\
\text { 'Naphtha' } \\
\text { From LiBr } \\
\text { Chiller } \\
\text { LIQUID }\end{array}$ & $\begin{array}{c}43 \\
\text { Waste } \\
\text { Water } \\
\text { LIQUID }\end{array}$ & $\begin{aligned} & 44 \\
& \text { 'Raw' } \text { LP } \\
& \text { Fuel } \text { Gas } \\
& \text { VAPOR }\end{aligned}$ \\
\hline & $\begin{array}{r}181 \\
27 \\
257 \\
64 \\
32 \\
0 \\
7 \\
27 \\
28 \\
4 \\
80 \\
4 \\
94 \\
662 \\
9784 \\
9391 \\
2454 \\
0 \\
0 \\
0 \\
0 \\
5\end{array}$ & $\begin{array}{r}0 \\
0 \\
0 \\
3 \\
3 \\
42473 \\
0 \\
0 \\
0 \\
0 \\
0 \\
0 \\
0 \\
0 \\
0 \\
0 \\
0 \\
0 \\
0 \\
0 \\
0 \\
0 \\
0 \\
0 \\
258\end{array}$ & $\begin{array}{r}0 \\
9 \\
3 \\
8 \\
102 \\
2 \\
0 \\
0 \\
1 \\
1 \\
0 \\
2 \\
0 \\
2 \\
12 \\
352 \\
6343 \\
29524 \\
0 \\
0 \\
0 \\
0 \\
0 \\
1\end{array}$ & & $\begin{array}{r}4571 \\
267412 \\
40538 \\
49207 \\
156 \\
19957 \\
19 \\
944 \\
1285 \\
1187 \\
67 \\
1276 \\
69 \\
1261 \\
3327 \\
2798 \\
0 \\
0 \\
0 \\
0 \\
0 \\
0 \\
0 \\
18\end{array}$ & $\begin{array}{r}70 \\
70 \\
14 \\
140 \\
8 \\
26 \\
0 \\
3 \\
10 \\
11 \\
2 \\
33 \\
1 \\
39 \\
320 \\
2108 \\
30 \\
0 \\
0 \\
0 \\
0 \\
0 \\
1\end{array}$ & $\begin{array}{r}0 \\
23 \\
5 \\
18 \\
499 \\
1 \\
0 \\
0 \\
0 \\
0 \\
0 \\
0 \\
0 \\
0 \\
0 \\
0 \\
0 \\
0 \\
0 \\
0 \\
0 \\
0 \\
5\end{array}$ & $\begin{array}{r}5 \\
437 \\
111 \\
590 \\
14 \\
88 \\
0 \\
14 \\
46 \\
48 \\
5 \\
101 \\
108 \\
108 \\
331 \\
193 \\
0 \\
0 \\
0 \\
0 \\
0 \\
0 \\
31\end{array}$ \\
\hline $\begin{array}{l}\mathrm{B} / \mathrm{HR} \\
\mathrm{BMOL} / \mathrm{HR} \\
\theta \mathrm{g} \mathrm{F}\end{array}$ & $\begin{array}{r}23103 \\
177 \\
100 \\
433\end{array}$ & $\begin{array}{r}42734 \\
2364 \\
0 \\
0\end{array}$ & $\begin{array}{r}36361 \\
111 \\
330 \\
100\end{array}$ & & $\begin{array}{r}394091 \\
15846 \\
70 \\
320\end{array}$ & $\begin{array}{r}2816 \\
36 \\
50 \\
333\end{array}$ & $\begin{array}{r}551 \\
29 \\
50 \\
333\end{array}$ & $\begin{array}{r}2128 \\
56 \\
110 \\
75\end{array}$ \\
\hline
\end{tabular}


Table 3.3 (Continued)

F-T Synthesis (Area 200) - Heat and Material Balances (4 of 6)

Mass Flow, Lb/hr

\begin{tabular}{|c|c|c|c|c|c|c|}
\hline stream \# & $\begin{array}{r}45 \\
\text { Naphtha } \\
\text { From Product } \\
\text { Fractionator } \\
\text { LIQUID }\end{array}$ & $\begin{array}{r}\text { Distillate } \\
\text { From Product } \\
\text { Fractionator } \\
\text { LIQUID }\end{array}$ & $\begin{array}{r}\text { Wax To } \\
\text { Hydrocrack } \\
\text { LIQUID }\end{array}$ & $\begin{array}{c}48 \\
\text { Waste } \\
\text { Water } \\
\text { LIQUID }\end{array}$ & 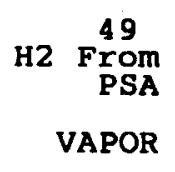 & $\begin{array}{r}50 \\
\text { Naphtha } \\
\text { From Wax } \\
\text { Hydrocracker } \\
\text { LIQUID }\end{array}$ \\
\hline & $\begin{array}{r}0 \\
0 \\
0 \\
0 \\
13 \\
0 \\
0 \\
0 \\
4 \\
5 \\
2 \\
47 \\
2 \\
67 \\
941 \\
17113 \\
379 \\
0 \\
0 \\
0 \\
0 \\
0 \\
0 \\
5\end{array}$ & $\begin{array}{r}0 \\
0 \\
0 \\
0 \\
22 \\
0 \\
0 \\
0 \\
0 \\
0 \\
0 \\
0 \\
0 \\
0 \\
0 \\
4960 \\
29888 \\
6467 \\
0 \\
0 \\
0 \\
0 \\
0\end{array}$ & $\begin{array}{r}0 \\
0 \\
0 \\
0 \\
0 \\
0 \\
0 \\
0 \\
0 \\
0 \\
0 \\
0 \\
0 \\
0 \\
0 \\
44 \\
6103 \\
29019 \\
0 \\
0 \\
0 \\
0 \\
0\end{array}$ & $\begin{array}{r}0 \\
0 \\
0 \\
9 \\
293 \\
0 \\
0 \\
0 \\
0 \\
0 \\
0 \\
0 \\
0 \\
0 \\
0 \\
0 \\
0 \\
0 \\
0 \\
0 \\
0 \\
0 \\
0 \\
0\end{array}$ & $\begin{array}{r}383 \\
54 \\
0 \\
0 \\
0 \\
0 \\
0 \\
0 \\
0 \\
0 \\
0 \\
0 \\
0 \\
0 \\
0 \\
0 \\
0 \\
0 \\
0 \\
0 \\
0 \\
0 \\
0\end{array}$ & $\begin{array}{r}0 \\
2 \\
1 \\
31 \\
3 \\
2 \\
0 \\
2 \\
6 \\
77 \\
2 \\
33 \\
5 \\
43 \\
1795 \\
2373 \\
0 \\
0 \\
5079 \\
1631 \\
0 \\
0 \\
16\end{array}$ \\
\hline $\begin{array}{l}\mathrm{LB} / \mathrm{HR} \\
\mathrm{LBMOL} / \mathrm{HR} \\
\operatorname{deg} \mathrm{F} \\
\mathbf{a}\end{array}$ & $\begin{array}{r}18578 \\
176 \\
100 \\
18\end{array}$ & $\begin{array}{r}41337 \\
209 \\
100 \\
18\end{array}$ & $\begin{array}{r}35166 \\
98 \\
350 \\
80\end{array}$ & $\begin{array}{r}303 \\
17 \\
110 \\
75\end{array}$ & $\begin{array}{l}437 \\
192 \\
110 \\
500\end{array}$ & $\begin{array}{r}11100 \\
107 \\
170 \\
22\end{array}$ \\
\hline
\end{tabular}


Table 3.3 (Continued)

F-T Synthesis (Area 200) - Heat and Material Balances (5 of 6)

Mass Flow, Lb/hr

Stream \#

\begin{abstract}
Distilate rom Wax
\end{abstract} Hydrocracker

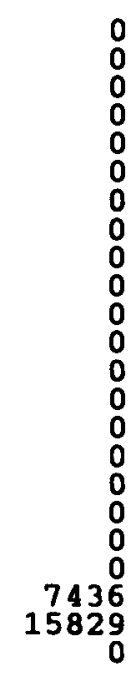

Components :

H2

N2

$\mathrm{CO} 2$

$\mathrm{H} 2 \mathrm{O}$

C24 4

C2H6

C3H6

C3H8

NC4 8

IC $4 \mathrm{H} 10$

NC4 410

C58

C6-C10

C20+ Wax

$300-350 \mathrm{HC}$

$350-500 \mathrm{HC}$

$500+\mathrm{HC}$

Oxygenater

Total Flow LB/HR

Total Flow LBMOL/HR

Temperature deg $F$

Preseure Pria
23265

99
360
32 $\begin{array}{lc}52 & 53 \\ \text { C4s } & \text { Hydrocrack }\end{array}$ From Wax Plant Overhead Hydrocracker To LP Fuel Gas

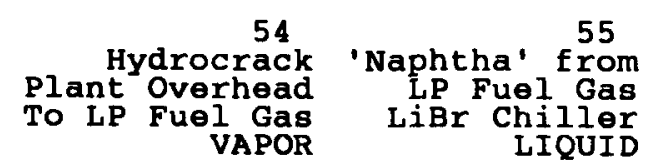

1220
21
100
30
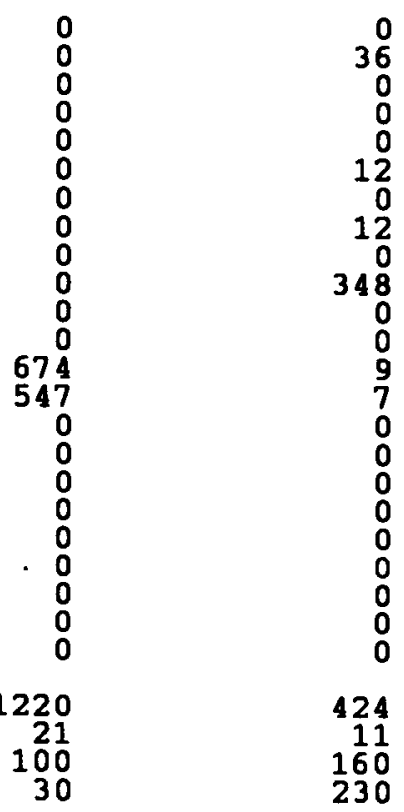

$\begin{array}{rr}0 & \\ 0 & 0 \\ 0 & 36 \\ 0 & 0 \\ 0 & 0 \\ 0 & 0 \\ 0 & 12 \\ 0 & 0 \\ 0 & 12 \\ 0 & 0 \\ 0 & 348 \\ 0 & 0 \\ 674 & 0 \\ 547 & 9 \\ 0 & 7 \\ 0 & 0 \\ 0 & 0 \\ 0 & 0 \\ 0 & 0 \\ 0 & 0 \\ 0 & 0 \\ 0 & 0 \\ 0 & 0 \\ 0 & 0 \\ 220 & 424 \\ 21 & 11 \\ 100 & 160 \\ 30 & 230\end{array}$

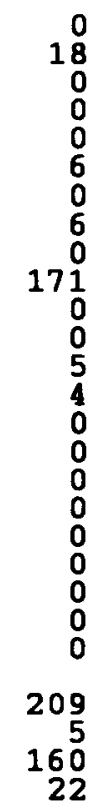

0
2
1
31
3
2
0
2
6
77
2
33
5
43
216
177
0
0
0
0
0
0
16
616
10
55
105 
F-T Synthesis (Area 200) $\frac{\text { Table } 3.3 \text { (Continued) }}{\text { - Heat and Material Balances (6 of 6) }}$

Mass Flow, Lb/hr

Stream \#

$\begin{array}{cr}\text { Total } & \text { Total } \\ \text { Naphtha } & \text { Distiliate } \\ \text { Production } & \text { Production } \\ \text { LIQUID } & \text { LIQUID }\end{array}$

Total
CAs
Production
LIQUID
Total
Production

LP Fuolal

Production

LIQUID

LIQUID

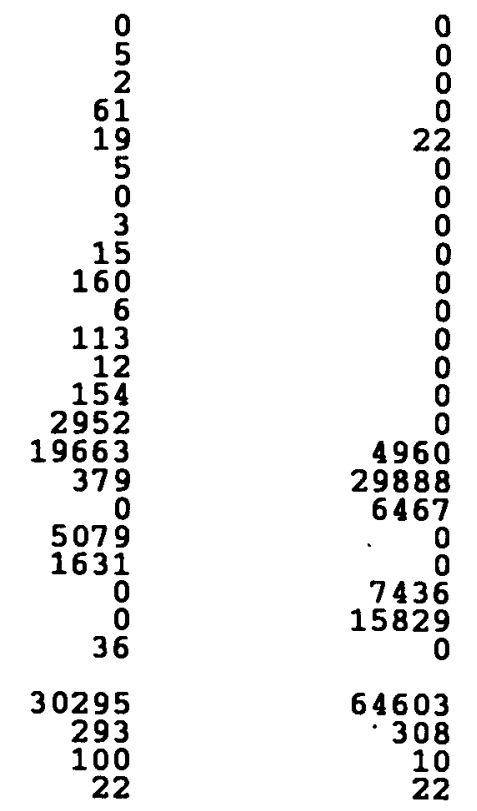

0
0
0
0
0
0
0
0
0
0
0
0
674
547
0
0
0
0
0
0
0
0
0
1220
21
100
50
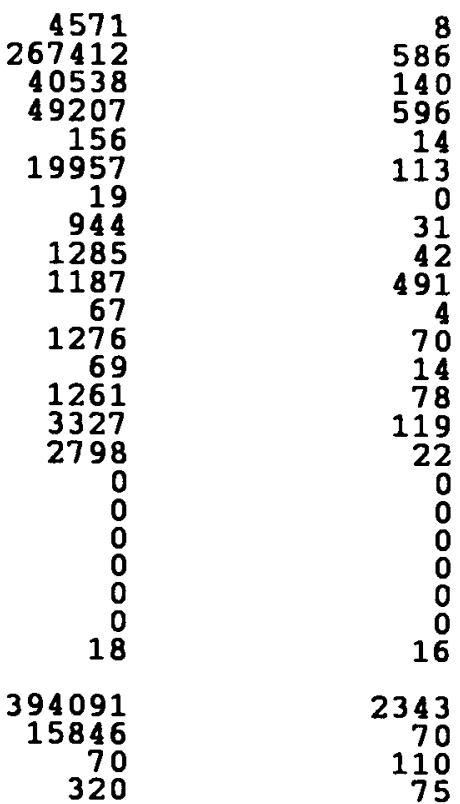

0
0
0
0
22
0
0
0
0
0
0
0
0
0
0
4960
29888
6467
0
0
7436
15829
0
64603
308
10
22

VAPOR

VAPOR

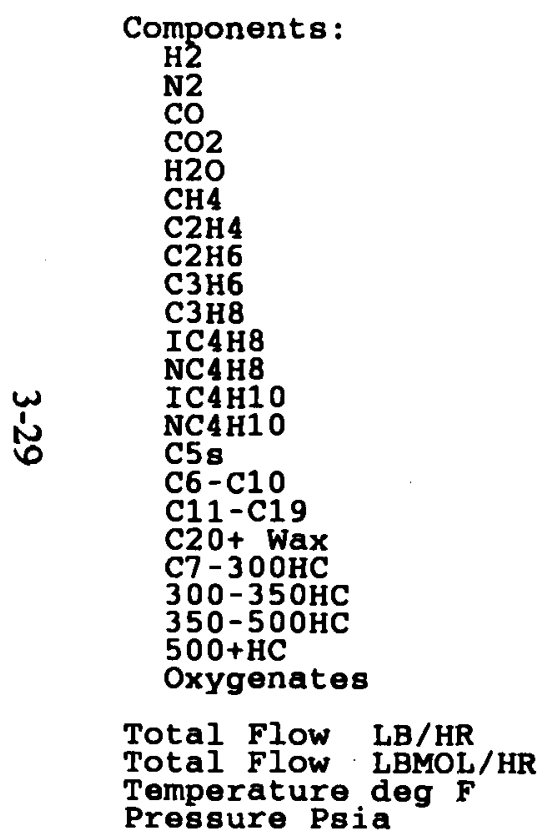




\subsection{Utility Balance Summary}

The overall utility balance for this once-through natural gas-based F-T facility is shown in Table 3.4. This table contains the utility consumptions and/or productions for each of the process plants in the two major processing areas as well as those for the offsite facilities. 
Table 3.4

Utility Summary (1)

\begin{tabular}{|c|c|c|c|c|c|c|c|c|c|c|c|c|c|}
\hline \multirow[t]{2}{*}{$\begin{array}{l}\text { Plant } \\
\text { No. }\end{array}$} & \multirow[t]{2}{*}{\begin{tabular}{|l} 
Plant \\
Deseription
\end{tabular}} & \multirow{2}{*}{$\begin{array}{l}\text { Lond } \\
\text { BHP }\end{array}$} & \multirow{2}{*}{$\begin{array}{l}\text { Power } \\
\text { kW }\end{array}$} & \multicolumn{4}{|c|}{ MP Staam, Mb/hr } & \multicolumn{6}{|c|}{ LP Stoam, Mb/hr } \\
\hline & & & & Produced & Consumed & Produced & Consumed & Produced & Consumed & Produced & Consumed & Produced & Consumed \\
\hline & Area 100 & & & & & & & & & & & & \\
\hline 101 & Air Separation & 92,747 & 76,846 & & & & & & 3.53 & & & & \\
\hline 102 & Autothermal Reforming & 903 & 748 & & & 327.90 & & & & & & & \\
\hline \multirow[t]{4}{*}{103} & CO2 Removal & 9,614 & 7,966 & & & & & & & & & & 129.00 \\
\hline & & & & & & & & & & & & & \\
\hline & Subtotal: & 103,264 & 85,560 & 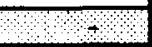 & + & $327 \% 90$ & + & & 3.53 & & & & 129.00 \\
\hline & Area 200 & & & & & & & & & & & & \\
\hline 201 & F-T Synthesis & 1,416 & 1,173 & & & & 3.80 & & & 605.00 & & & 10.60 \\
\hline 202 & Hydrogen Recovery & 1.336 & 1,107 & & & & & & & & & & \\
\hline 203 & Product Fractionation & 37 & 31 & & & & & & 7.20 & & & & \\
\hline \multirow[t]{5}{*}{204} & Wax Hydrocracking & 925 & 766 & & & & 8.20 & & 2.60 & & & & \\
\hline & & & & & & & & & & & & & \\
\hline & Subtotal: & 3,714 & 3,077 & & 3 & 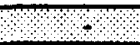 & 12.00 & & 9.80 & 605.00 & + & & 10.60 \\
\hline & & & & & & & & & & & & & \\
\hline & Offsitos & & & & & & & & & & & & \\
\hline 19 & Relief and Blowdown & 4 & 3 & & & & & & & & & & \\
\hline 20 & Tankage & 15 & 13 & & & & & & & & & & \\
\hline 21 & Intercon. Piping System & & & & & & & & & & & & \\
\hline 22 & Product Shipping & 270 & 224 & & & & & & & & & & \\
\hline 23 & Tank Car/Truck Loeding & 7 & 6 & & & & & & & & & & \\
\hline 25 & Cot/Chem. Handing & 11 & 9 & & & & & & & & & & \\
\hline \multirow[t]{4}{*}{31} & 50 Psig Flash Drum & & & & & & & & & & & 2.10 & \\
\hline & Combined Cycle Plant & & $(183,300)$ & & 315.90 & & & & 591.70 & & & 152.50 & \\
\hline & BFW \& Cond Pumpe & 1,764 & 1,461 & & & & & & & & & & \\
\hline & Steam Superheaters & & & 315.90 & & & 315.90 & 605.00 & & & 605.00 & & \\
\hline 32 & Row/Cooling/Pot Water & 7,979 & 6,611 & & & & & & & & & & \\
\hline 33 & Fire Protection Syatem & 9 & 8 & & & & & & & & & & \\
\hline 34 & Sewrga/Effi. Treatment & 1,040 & 862 & & & & & & & & & & \\
\hline 35 & Instrument/Plant Air & 615 & 510 & & & & & & & & & & \\
\hline 36 & Purge/Plush Oil System & & & & & & & & & & & & \\
\hline 37 & Solid Weste Manegement & 10 & 9 & & & & & & & & & & \\
\hline 40 & General Site & & & & & & & & & & & & \\
\hline 41 & Buildinges & 572 & 474 & & & & & & & & & & \\
\hline 42 & Telecommunications & 3 & 2 & & & & & & & & & & \\
\hline \multirow[t]{2}{*}{ Other } & Miscollaneous & 452 & 375 & & & & & & & & & & 15.00 \\
\hline & Subtotal & 12,751 & $(172,735)$ & 316 & 315.90 & ధి & 315.90 & 60500 & 591.70 & & 605.00 & 154.60 & 15.00 \\
\hline TOTAL & & 119,729 & (84,098) & 316 & 316 & 328 & $\mathbf{3 2 8}$ & 8605 & 605 & 605 & 605 & 165 & 155 \\
\hline
\end{tabular}


Table 3.4 (Continued)

Utility Summary (2)

\begin{tabular}{|c|c|c|c|c|c|c|c|c|c|c|c|}
\hline \multirow{2}{*}{$\begin{array}{l}\text { Plant } \\
\text { No. }\end{array}$} & \multirow{2}{*}{$\begin{array}{l}\text { Plant } \\
\text { Deseription }\end{array}$} & \multicolumn{3}{|c|}{ Condansato, $\mathrm{Mlb} / \mathrm{hr}$} & \multicolumn{2}{|c|}{ Cond, $\mathrm{Mbb} / \mathrm{hr}$} & \multicolumn{2}{|c|}{ Wator } & \multicolumn{2}{|c|}{ Cooling,MMBtu/hr } & \multirow{2}{*}{$\begin{array}{l}\text { Fuel, } \\
\text { MMBtu/hr } \\
\text { Total Fired }\end{array}$} \\
\hline & & 635 Psig & 150 Psig & 50 Psig & $\begin{array}{l}\text { BFW } \\
\text { Mlb/hr }\end{array}$ & $\begin{array}{l}\text { Blowdown } \\
\text { Mlb/hr }\end{array}$ & $\begin{array}{l}\text { Process } \\
\text { Mlb/hr }\end{array}$ & $\begin{array}{l}\text { C.W. Circ. } \\
\text { GPM }\end{array}$ & Air & & \\
\hline & Aroa 100 & & & & & & & & & & \\
\hline 101 & Air Soparation & & (3.5) & & & & & $24,740.12$ & & 284.50 & \\
\hline 102 & Autothermal Reforming & & & & 334.5 & 6.6 & & & 301.10 & & 105 \\
\hline \multirow[t]{4}{*}{103} & CO2 Removal & & & $(129.0)$ & & & & $3,739.28$ & 100.00 & 43.00 & \\
\hline & & & & & & & & & & & \\
\hline & Subtotal: & & $(3,5)$ & $(1290)$ & 3345 & 6.6 & & $28,479.40$ & 401,10 & 327.50 & 105 \\
\hline & Area 200 & & & & & & & & & & \\
\hline 201 & F-T Synthesis & (3.8) & & (10.6) & 623.2 & 12.4 & & $2,487.06$ & 98.20 & 28.60 & 1 \\
\hline 202 & Hydrogen Recovery & & & & & & & 510.46 & & 5.87 & \\
\hline 203 & Product Fractionation & & (7.2) & & & & & 765.25 & 12.00 & 8.80 & 23 \\
\hline \multirow[t]{5}{*}{204} & Wax Hydrocracking & (8.2) & (2.6) & & & & & 460.30 & 32.00 & 5.80 & 11 \\
\hline & & & & & & & & & & & \\
\hline & Subtotals & 81201 & $(9.8)$ & $(10.6)$ & 8232 & 124 & & 4.223 .06 & $\mathbf{1 4 2 . 2 0}$ & 49.07 & 35 \\
\hline & & & & & & & & & & & \\
\hline & Offites & & & & & & & & & & \\
\hline 19 & Relief and Blowdown & 12.0 & (12.0) & & & & & & & & \\
\hline 20 & Tankage & & & & & & & & & & \\
\hline 21 & Intercon. Piping System & & & & & & & & & & \\
\hline 22 & Product Shipping & & & & & & & & & & \\
\hline 23 & Tank Car/Truck Loading & & & & & & & & & & \\
\hline 25 & Cat./Chem. Handing & & & & & & & & & & \\
\hline \multirow[t]{4}{*}{31} & 50 Psig Flash Drum & & 25.3 & (23.2) & & & & & & & \\
\hline & Combined Cycle Plant & & & $(1,064.6)$ & 315.7 & 6.2 & & $65,811.33$ & & 756.80 & 870 \\
\hline & BFW \& Cond Pumpe & & & & & & & & & & \\
\hline & Steam Superheaters & & & & & & & & & & 90 \\
\hline 32 & Renw/Cooling/Pot. Water & & & & $(31.0)$ & & & & & & \\
\hline 33 & Fire Protection Syetem & & & & & & & & & & \\
\hline 34 & Sewage/Effi. Treatment & & & & & & & & & & \\
\hline 35 & Instrument/Plant Air & & & & & & & & & & \\
\hline 36 & PurgelPlush Oil System & & & & & & & & & & \\
\hline 37 & Solid Waste Management & & & & & & & & & & \\
\hline 40 & General Site & & & & & & & & & & \\
\hline 41 & Buildinges & & & & & & & & & & \\
\hline 42 & Telecommunications & & & & & & & & & & \\
\hline \multirow[t]{2}{*}{ Other } & Miscollaneous & & & $(15.0)$ & & & & & & & \\
\hline & Subtotal & 12.0 & 13.3 & $(1,1028)$ & 2047 & 62 & & $65,811,33$ & 0 & 756.80 & 860 \\
\hline TOTAL & $\downarrow$ & $+\square$ & 10.01 & $(1,242.4)$ & 1,2424 & 25.2 & & 98,514 & 543 & 1,133 & 1,100 \\
\hline
\end{tabular}




\subsection{Overall Steam and Condensate Balance}

The overall plant steam condensate balance for this once-through natural gas-based F-T facility is shown schematically in Figure 3.2. All flow rates shown in Figure 3.2 are expressed in thousands of pounds per hour. 


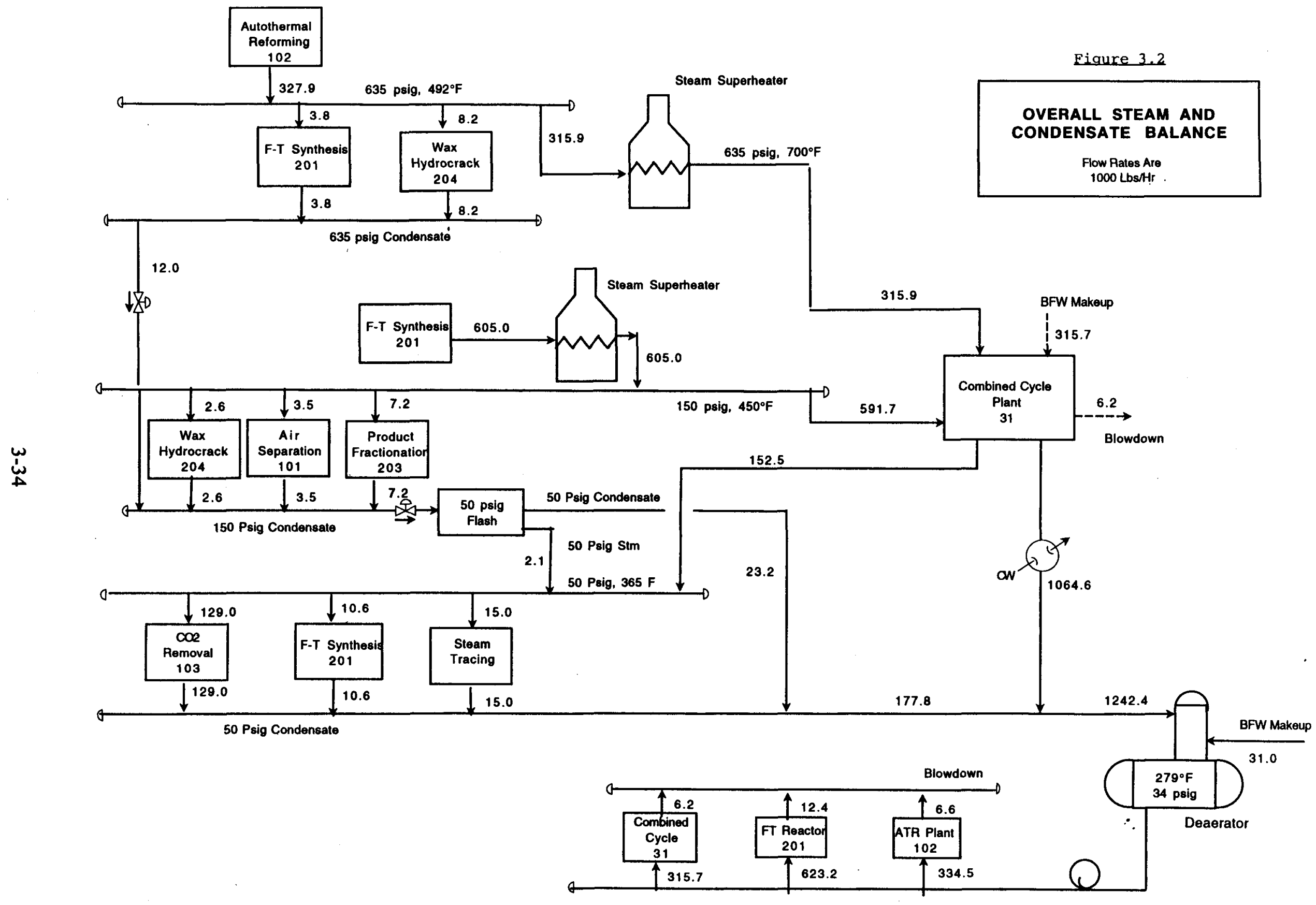




\section{PROCESS DESCRIPTIONS - AREA 100, SYNGAS PREPARATION AREA}

Area 100 is the syngas preparation area. For this once-through natural gas case, this area consists of the following three plants as shown in Figure 3.1.

Plant 101 Air Separation Plant

Plant 102 Autothermal Reforming Plant

Plant $103 \quad \mathrm{CO}_{2}$ Removal and Recycle Plant

The sulfur removal, syngas cooling and heat recovery/steam generation are parts of Plant 102, the Autothermal Reforming Plant.

Syngas is generated in the autothermal reforming plant using enriched air containing 40 mole \% oxygen rather than 99.5 mole \% oxygen as was used in the previous natural gas study. ${ }^{2}$ The use of enriched air for syngas generation is a requirement of Syncrude Technology, Incorporated's proprietary Fischer-Tropsch synthesis technology.

Plant 101, the Air Separation Plant, contains an inlet air compressor. The cryogenic air separation portion of Plant 101 produces a 95 mole \% oxygen stream which when blended with the output from the air compressor produces an enriched air stream containing $\mathbf{4 0}$ mole \% oxygen that is sent to Plant 102, the Autothermal Reforming Plant.

Plant 102, the Autothermal Reforming Plant, first removes trace amounts of sulfur containing compounds from the natural gas by reaction with zinc oxide. The desulfurized natural gas then is mixed with the enriched air, recycle $\mathrm{CO}_{2}$ and steam before entering the autothermal reformer reactor where it is converted to syngas. The hot syngas product stream is cooled by feed/effluent heat exchange, steam generation, and exchange with ambient air. It is then flashed to condensed and remove water before going on to Plant 103, the $\mathrm{CO}_{2}$ Removal and Recycle Plant.

Plant 103, the $\mathrm{CO}_{2}$ Removal and Recycle Plant, is a standard MDEA absorption plant which removes $\mathrm{CO}_{2}$ from a portion of the cooled syngas stream leaving Plant 102 and recycles it back to Plant 102 . The lean syngas after $\mathrm{CO}_{2}$ removal is mixed with that bypassing Plant 103 to produce a combined synthesis gas steam having a molar $\mathrm{H}_{2} / \mathrm{CO}$ ratio of 2.01 which is sent to Area 200 for Fischer-Tropsch synthesis.

The following three subsections give a more detailed description on each of the three process plants in Area 100, the Syngas Preparation Area. 


\subsection{Plant 101 - Alr Separation Plant}

Plant 101, the Air Separation Plant, provides the required enriched air feed to Plant 102, the Autothermal Reforming Plant, for syngas generation. The air separation portion of Plant 101 is a standard cryogenic air separation plant, similar to that used in the previous natural gas and coal liquefaction studies. ${ }^{1,2}$ However, it has been modified to produce a lower purity oxygen product stream containing 95 mole \% oxygen rather than the 99.5 mole \% oxygen concentration used in the previous cases. This was done to obtain a small reduction in cost. In addition, this plant includes an air compressor so that the final enriched air product leaving this plant contains 40 mole $\%$ oxygen.

\section{Design Basis and Considerations}

The cryogenic air separation plant is a single train with a capacity of about 1,550 STPD of 95 mole \% pure oxygen. The design incorporates a backup system including a liquid oxygen storage capacity of 1,550 tons, which is equivalent to one days production, and a gaseous oxygen storage of 43 tons, which is equivalent to 40 minutes of production. This backup oxygen storage system protects the facility from an unscheduled shutdown of one day or less.

\section{Process Description (PFD 101-B-01)}

A process simplified flow diagram of Plant 101, the Air Separation Plant, is shown in PFD 101-B-01.

In the air separation section, ambient air is filtered and compressed in a two-stage axial centrifugal compressor with interstage cooling. The air from the final stage of compression enters a direct contact aftercooler where it contacts cooling water and chilled cooling water in two separate packed sections.

The cooled air from the top of the aftercooler has lost the majority of its ambient water vapor. Removal of the residual water vapor, carbon dioxide and other atmospheric contaminants occurs in the molecular sieve adsorbers. Two vessels containing the adsorbent are used in a cyclic process. While one vessel is on line purifying the incoming air, the other vessel first is heated with dry waste nitrogen gas to remove the adsorbed contaminants and then cooled to the operating temperature before being placed back in service. The regeneration heater uses high pressure steam to raise the temperature of the regeneration gas to the proper level.

The dry air enters the "cold box" where it is cooled to cryogenic temperature in the main heat exchangers and is separated into oxygen and nitrogen by cryogenic distillation. Final cooling is by expansion. The oxygen stream is further purified in a argon column to 95 mole \%. The main heat exchangers are brazed aluminum, multipass, plate-fin 
units in which the entering air is cooled against the cold oxygen and nitrogen streams leaving the distillation columns.

The oxygen product stream leaving the cryogenic separation section is warmed in the main heat exchangers and compressed to final delivery pressure in a centrifugal compressor. In order to insure a continuous supply of oxygen, backup storage systems are included in the design. A portion of the oxygen produced by distillation is withdrawn as liquid and stored at low pressure in an insulated tank. In addition to liquid oxygen storage, a high pressure gaseous oxygen storage tank also is provided. This tank is periodically filled by pumping liquid oxygen to over 2000 psia, vaporizing the liquid and storing it at pressure at ambient temperature. Oxygen from the gaseous storage tank is always available to meet a shortfall in supply pressure regardless of the cause. Sufficient gaseous storage capacity is provided to allow time to cool the main liquid oxygen supply pumps to operating temperature. Oxygen supply is then provided by pumping liquid oxygen to supply pressure and vaporizing it with steam.

Compressed air is mixed with the 95 mole \% oxygen produced by the air separation section to produce an enriched air stream containing 40 mole \% oxygen that is sent to Plant 102, the Autothermal Reforming Plant, for syngas generation.

\section{TechnologyNendor Selection}

There are at least four major vendors who can supply turnkey air separation plants of this size: Liquid Air Engineering, Airco, Air Products, and Union Carbide. Each design has a slightly different power requirement and capital cost. The Air Products design was selected for this study as with the previous two studies.

The ISBL cost of this plant is based on a January, 1997 quotation from Air Products and Chemicals, Inc. This quotation is without the two air compressors. For costing purposes, the two separate air compressors were combined into a single, seven-stage compressor with intercoolers with the feed to the air separation section being withdrawn after the fourth stage. This single 85,000 BHP air compressor is directly driven by a gas turbine, which is shown as part of Plant 31, the Combined-Cycle Power Generation Plant. The exhaust gases leaving the gas turbine are sent to the Heat Recovery Steam Generation (HRSG) system to produce steam for power generation. 


\subsection{Plant 102 - Autothermal Reforming Plant}

The objective for Plant 102, the Autothermal Reforming Plant, is to provide a syngas with a $\mathrm{H} 2 / \mathrm{CO}$ ratio of about $2.0(2.0+1-0.1)$ as specified by Syncrude Technology Incorporated for Fischer-Tropsch synthesis in Plant 201. This objective is accomplished using a 40 mole \% enriched air-fired autothermal reformer (ATR) operating with $\mathrm{CO}_{2}$ recycle.

STI agreed to use this ATR design with $\mathrm{CO}_{2}$ recycle for syngas generation.

\section{Design Basis and Considerations}

Besides the syngas $\mathrm{H}_{2} / \mathrm{CO}$ ratio, the $\mathrm{H} 2$ and $\mathrm{CO}$ partial pressures entering the FischerTropsch slurry-bed reactors also were governing factors in the design of the syngas preparation area. Autothermal reforming calculations/trade-off studies were carried out to determine that the syngas requirements could best be met with an ATR using 40 mole $\%$ enriched air operating with $\mathrm{CO}_{2}$ recycle. Inputs from various ATR technology vendors were solicited to confirm Bechtel's design calculations, and to provide both utility and cost estimates. Lurgi Corporation responded by providing a 'black-box' design with an overall cost and utility estimate.

Bechtel carried out detailed heat and material balances for this plant to obtain the stream flow rates and conditions shown in Table 3.1. An equipment list was generated from which a cost breakdown was obtained to verify Lurgi's cost estimate.

The ATR reactor is designed to operate at a reasonable $\mathrm{O}_{2} / \mathrm{C}$ molar ratio 0.7 to keep the maximum adiabatic flame temperature below $4000^{\circ} \mathrm{F}$, and at a steam/carbon molar ratio of 0.6 to avoid potential soot formation.

\section{Process Description (PFD 102-B-01)}

A simplified process flow diagram of Plant 102, the Autothermal Reforming Plant, is shown in PFD 102-B-01.

The natural gas feed is heated by heat exchange in 102E-2 before being fed to a fired feed preheater, 102F-1. The heated natural gas is then desulfurized in one of the two mixed CoMo/ZnO fixed-bed reactors (102C-1ABB). Sulfur is removed to the less than $0.1 \mathrm{ppm}$ by volume as required for the subsequent Fischer-Tropsch synthesis. The CoMo/ZnO reactor beds are arranged in parallel to allow for continuous desulfurization within one reactor bed while the catalyst/absorbent in the other bed is being regenerated. 
The desulfurized natural gas is further heated in the fired feed preheater, 102F-1. The heated natural gas is then mixed with heated enriched air from Plant 101 , recycle $\mathrm{CO}_{2}$ from Plant 103 , and superheated $650 \mathrm{psia} / 1000^{\circ} \mathrm{F}$ steam before going to the $102 \mathrm{R}-1$ autothermal reforming reactor. The autothermal reforming reactor effluent is cooled in a series of heat exchangers, 102E-1A, 102E-1B and 102E-2, to produce 650 psia steam and preheat the incoming natural gas feed. Final cooling of the syngas to $150^{\circ} \mathrm{F}$ is accomplished in the 103E-3 air cooler. The cooled syngas goes to flash drum 102C-3 where the condensed water is separated from the product syngas which is sent to Plant 103, the CO2 Removal Plant.

The syngas product has a molar $\mathrm{H} 2 / \mathrm{CO}$ ratio of 2.01 .

\section{TechnologyNendor Selection}

Plant 102, the Autothermal Reforming Plant, is based on a Lurgi design and cost estimate. Lurgi provided a quotation with utility requirements for the design in July, 1996 which calls for a single ATR train with $\mathrm{CO}_{2}$ recycle at a total installed cost of 38.4 MM\$. This cost includes the ATR reactor, fired feed heater, waste heat recovery system, $\mathrm{MDEA} \mathrm{CO} \mathrm{C}_{2}$ removal section, and $\mathrm{CO}_{2}$ recycle compressor.

Bechtel carried out detailed heat and material balances for this plant to verify Lurgi's cost estimate and to separate the cost of the autothermal reforming plant from the $\mathrm{CO}_{2}$ Removal and Recycle Plant. The total estimated cost for Plant 102, the Autothermal Reforming Plant, is $22.8 \mathrm{MM} \$$, excluding the MDEA plant and the $\mathrm{CO}_{2}$ recycle compression costs. 


\subsection{Plant $103-\mathrm{CO}_{2}$ Removal and Recycle Plant}

Plant 103, the $\mathrm{CO}_{2}$ Removal and Recycle Plant, is required to provide a $\mathrm{CO}_{2}$ recycle stream back to Plant 102 to control the equilibrium $\mathrm{H}_{2}$ and $\mathrm{CO}_{2}$ production in the Autothermal Reforming Plant.

\section{Design Basis and Considerations}

In order for the autothermal reformer to produce the required $\mathrm{H}_{2} / \mathrm{CO}$ ratio in the product syngas, about $66 \%$ of the $\mathrm{CO}_{2}$ leaving Plant 102 is recovered, compressed and recycled back to the autothermal reformer reactor.

\section{Process Description (PFD 103-B-01)}

A simplified process flow diagram of Plant 103 , the $\mathrm{CO}_{2}$ Removal Plant, is shown in PFD 103-B-01. Also shown in this diagram are two extra pieces of equipment which are not part of a typical $\mathrm{CO}_{2}$ Removal Plant, the $103 \mathrm{E}-1$ syngas cooler and the $103 \mathrm{~K}-1 \mathrm{CO}_{2}$ recycle compressor.

PFD 103-B-01 is a flow diagram of a typical amine absorption plant. The syngas product stream from Plant 102, stream 12A, is split so that only a portion of it goes to the $\mathrm{CO}_{2}$ Removal Plant. The syngas going to Plant 103 , stream $12 \mathrm{C}$, is cooled to $110^{\circ} \mathrm{F}$ in exchanger 103E-1 and sent to the feed gas knock out drum before going to the amine absorber column for $\mathrm{CO}_{2}$ removal. To ensure an amine-free vapor product, the absorber overhead vapor is water washed in a scrubber. The scrubber liquid is returned to the rich-amine knock out drum. The washed, lean syngas stream leaving the scrubber, stream 22, is combined with syngas stream 12B, which bypasses Plant 103 , to produce stream 23, the syngas product stream which is sent to Area 200 for Fischer-Tropsch synthesis.

The rich-amine solution from the bottom of the amine absorber column is flashed in a flash drum. It is then heated by exchange with the lean-amine solution before being sent to one of the two amine regenerators. Because of the high reboiler duty required by the high $\mathrm{CO}_{2}$ removal rate, two parallel amine regenerators are required. Each regenerator has four reboilers associated with it. The regenerated lean-amine solution from these two regenerators is cooled by heat exchange with rich-amine before being going to a common amine storage tank. The lean-amine then is pumped from this tank, cooled and sent back to the absorber. A portion, approximately $10 \%$, of the cooled leanamine solution is filtered and returned to the storage tank. 
The $\mathrm{CO}_{2}$ which is removed from the syngas and leaves the amine regenerators is compressed in the 103K-1 $\mathrm{CO}_{2}$ compressor and sent back to Plant 102, the Autothermal Reforming Plant, to control the $\mathrm{CO}_{2}$ production during syngas generation.

TechnologyNendor Selection

There are several proprietary amine solvents such as Gas/Spec (Dow Chemical), UCARSOL (Union Carbide), Amine Guard (UOP), Flexsorb (Exxon) etc., which contain high concentrations of either MDEA or glycol amines plus proprietary additives. There is not much difference between these processes from a heat requirement or cost standpoint. The major difference is in the corrosion protection schemes.

Dow's Gas/Spec process, which uses a 50\% MDEA solution plus proprietary additives, was selected as a representative process for the process design.

The cost estimate for Plant 103 is 13.4 MM\$. This gives a total cost for the combined cost of Plants 102 and 103 of $36.2 \mathrm{MM} \$$ as compared to Lurgi's 'black-box' quotation of 38.4 MM\$. 


\section{PROCESS DESCRIPTION - AREA 200, FISHER-TROPSCH SYNTHESIS AND PRODUCT UPGRADING}

Area 200 is the Fischer-Tropsch synthesis and product upgrading area. Figure 3.1 shows a simplified block flow diagram of this area. It consists of the following four plants:

Plant 201 Fischer-Tropsch Synthesis Plant

Plant 202 Hydrogen Recovery Plant

Plant 203 Hydrocarbon Recovery Plant

Plant 204 Wax Hydrocracking Plant

This Area $\mathbf{2 0 0}$ is significantly different from the Fischer-Tropsch synthesis areas in either of the two previous studies. ${ }^{1,2}$ In those studies, a recycle design was used to obtain maximum liquids yields and minimize the co-production of power. Thus, the Fischer-Tropsch synthesis area contained a $\mathrm{CO}_{2}$ removal plant and a recycle compression and dehydration plant preceding the hydrocarbon recovery plant which, using refrigeration, recovered LPG in addition to all the heavier hydrocarbons. The removal of these materials from the F-T synthesis loop is necessary in order to minimize the amount of unconverted syngas being recycled and both equipment and operating cost associated with the recycle loop.

In the coal Baseline F-T study ${ }^{1}$, the syngas preparation area produced a syngas with a molar $\mathrm{H}_{2} / \mathrm{CO}$ ratio of about 0.7 , and a F-T synthesis design based on iron-based catalysts was used to promote in-situ water-gas shift activity. In that design, the produced F-T liquids were more olefinic and hydrotreating was required to stabilize them for shipment. Thus, a large hydrogen recovery plant was needed to supply the hydrogen required by the three hydroprocessing units in the product upgrading and refining area.

In the previous natural gas F-T study ${ }^{2}$, the syngas preparation area produced a syngas with a molar $\mathrm{H}_{2} / \mathrm{CO}$ ratio of about 2.0, and consequently, a cobalt-based $\mathrm{F}$-T synthesis catalyst with limited water-gas shift activity was used for the design. The recycle loop is somewhat smaller because only the hydrogen recovered from the hydrogen recovery plant is recycled back to the F-T synthesis reactors. Furthermore, the liquid products are less olefinic, and consequently, less hydrogen is required by the three hydroprocessing units in the product upgrading and refining area.

In the present once-through natural gas F-T design, an enriched air-blown autothermal reformer is used for syngas generation. The unconverted syngas leaving the secondstage, F-T synthesis reactor contains a significant amount of nitrogen which essentially precludes a recycle design of which the recovery of the $\mathrm{C} 3$ and $\mathrm{C} 4$ hydrocarbons from the unconverted syngas would have been more difficult and expensive. Thus, a less comprehensive hydrocarbon recovery plant is used which does not recover LPG. 
However, a $\mathrm{LiBr}$ absorption chiller is used to recover additional hydrocarbons from the unconverted syngas leaving the Fischer-Tropsch Synthesis Plant before it was sent to the high pressure fuel gas header.

The only product upgrading step in this design is a small wax hydrocracking plant which is included in this area. In the previous two studies, the wax hydrocracking plant was part of a separate and more extensive product upgrading and refining area which produced high quality gasoline and distillate blending components. In this case, the objective is only to produce a stable, shippable product which can be upgraded to liquid transportation fuels elsewhere, such as in a conventional petroleum refinery.

A small hydrogen recovery plant supplies the hydrogen needs of the wax hydrocracking plant by recovering hydrogen from a small portion of the syngas generated in the syngas preparation area. In contrast to the two previous designs, the feed to the hydrogen recovery plant is fresh syngas from Area 100 instead of the unconverted syngas leaving the hydrocarbon recovery plant. This design change was required because the hydrogen concentration in the unconverted syngas is too low for effective, economic hydrogen recovery.

The following four subsections give a more detailed description on each of the four process plants in Area 200, the Fischer-Tropsch Synthesis and Product Upgrading Area. 


\subsection{Plant 201 - Fischer-Tropsch Synthesis Plant}

The principle function of this plant is to convert the syngas produced in Area 100 into hydrocarbon products using cobalt catalyzed, slurry phase Fischer-Tropsch reactors. The reactor section of this plant consists of two first-stage slurry-bed reactors feeding a single second-stage slurry-bed reactor. There is no recycle of unconverted syngas back to the Fischer-Tropsch synthesis reactors.

\section{Design Basis and Considerations}

A total of three slurry-bed, F-T synthesis reactors are used to process the syngas from Area 100. These reactors are arranged in a single train consisting of two first-stage slurry-bed reactors feeding a single second-stage slurry-bed reactor. The unconverted syngas leaving the first-stage reactors is cooled to $150^{\circ} \mathrm{F}$ and flashed to condense and remove water and hydrocarbons before being reheated and fed to the single secondstage reactor. The CO conversion in both first-stage reactors as well as that in the second-stage reactor are both about $61 \%$.

Reactor sizing is based on the kinetic equations supplied by STI for the syngas composition generated in Area 100. The reactor design is that developed by Bechtel and used in the previous studies. ${ }^{3}$ In this design, the heat generated by the F-T synthesis reaction is removed by generation of 150 psig saturated steam in bayonet tubes suspended from a double tubesheet inside the reactor.

All three Fischer-Tropsch slurry reactors are about 19-feet in diameter and about 65feet in height. Each of the two parallel first-stage reactors contain about 2,000 bayonet tubes having a 1.5-inch OD for steam generation. The second-stage reactor contains 1470 of the same size steam tubes. Design superficial gas velocity in each of the two first-stage reactors is $11.8 \mathrm{~cm} / \mathrm{sec}$, and in the second-stage reactor, it is $14.5 \mathrm{~cm} / \mathrm{sec}$.

Catalyst replacement is estimated at $0.01 \%$ per hour of total catalyst inventory, which corresponds to an average catalyst life of about 1.2 years. This catalyst life is about twice that of the iron-based catalyst which is used in the coal Fischer-Tropsch Baseline design.

\section{Process Description (PFD 201-B-01,02,03)}

The process flow diagrams for Plant 201, F-T synthesis, are shown in PFD 201-B-01, PFD 201-B-02, and PFD 201-B-03.

As shown in PFD 201-B-01, the syngas from Plant 202 after hydrogen recovery is heated with steam to $350^{\circ} \mathrm{F}$ before entering the two parallel first-stage, F-T synthesis 
reactors (201C-3A and 201C-3B) through a cylindrical gas distributor. In the first-stage F-T reactors, the syngas bubbles upward through the catalyst/wax slurry, dissolves in the slurry phase, and is converted into hydrocarbon products at the catalyst interface. The slurry consists of solid catalyst particles suspended in the non-vaporizable portion of the liquid product (i.e. the wax). The heat of reaction is removed by the generation of 150 psig saturated steam at $372^{\circ} \mathrm{F}$ within the steam tubes.

The overhead vapor stream leaving the first-stage reactors goes through cyclone separators 201T-1A and 201T-1B to disengage any liquid carryover before being cooled to $150^{\circ} \mathrm{F}$ in exchangers $201 \mathrm{E}-9$ and $201 \mathrm{E}-10$. This cooled three-phase mixture is separated into an unconverted syngas stream, a liquid hydro- carbons stream, and a water stream in 201C-16. The liquid hydrocarbons stream is sent directly to Plant 204, the hydrocarbon recovery plant, and the water steam goes to water treatment.

The vapor stream is heat-exchanged to $350^{\circ} \mathrm{F}$ before entering the single second-stage, F-T synthesis reactor, $201 \mathrm{C}-3 \mathrm{C}$, through a cylindrical gas distributor. Also, in this F-T reactor, the syngas bubbles upward through the catalyst/wax slurry, dissolves in the slurry phase, and is converted into hydrocarbon products at the catalyst interface. The slurry consists of solid catalyst particles suspended in the non-vaporizable portion of the liquid product (i.e. the wax). The heat of reaction also is removed by the generation of 150 psig saturated team at $372^{\circ} \mathrm{F}$ within the steam tubes.

Liquid wax streams are withdrawn from all three F-T reactors at reactor conditions through STI's proprietary slurry valve which supposedly produces a catalyst free hydrocarbon stream. Without knowledge of how this slurry valve operates, hydroclones also are included to aid with producing a catalyst free product. Thus, upon leaving the reactors, the liquid wax passes through hydroclones. (201T-2A, 201T-2B and 201T-2C) to produce a 'catalyst free' overflow stream and a 'catalyst rich' underflow stream.

The overflow from the top of the hydroclones is passed through a valve to reduce its pressure to 86 psia (PFD 201-B-02) and sent to a product separator (201C-8). The pressure reduction causes dissolved gases to separate from the liquid product stream. The vapors from $201 \mathrm{C}-8$ are cooled to $100^{\circ} \mathrm{F}$ in exchanger $201 \mathrm{E}-4$ to recover additional liquid. The vapors from $201 \mathrm{C}-9$ are sent to fuel.

The liquids from 201C-8 and 201C-9 are sent to the F-T liquid wax intermediate hold tank, 201C-10, which serves as the feed drum for the wax clarifying, catalyst recovery filters, 201T-4A and 201T-4B. These are enclosed washing type cake filters. The clear wax stream is sent to Plant 203, the Hydrocarbon Recovery Plant. The underflow catalyst-wax slurry stream from the filters $201 T-4 A, B$ is mixed with make-up wax from Plant 203 and recycled back to the F-T reactors.

A portion of the catalyst is removed from the system as spent catalyst. About 600 pounds per day of catalyst are removed for disposal to counteract the catalyst makeup and maintain a constant catalyst inventory in the reactors. The spent catalyst train 
consists of an intermediate mixing tank, 201C-15, two catalyst-wax recovery filters, $201 \mathrm{~T}-8$, and a Holo-Flite slurry heated screw conveyor and solvent recovery system, 201T-9. Naphtha from downstream processing is used for mixing and recovery of wax left in the catalyst cake. It is stripped off from the clean spent catalyst in the Holo-Flite. The recovered naphtha is recycled.

The design of the F-T catalyst separation and recovery system is not very rigorous. A proven catalyst separation method for cobalt Fischer-Tropsch catalyst from which to base the design is not readily available. The selected scheme represents only a best engineering estimate based on current knowledge of the catalyst system, its characteristics, and available commercial separation equipment.

As shown in PFD 201-B-01, the overhead vapor stream from the second-stage F-T reactor is passed through a cyclone separator, 201T-1C, to disengage any liquid carryover. The vapor stream then is cooled to $100^{\circ} \mathrm{F}$ in heat exchanger $201 \mathrm{E}-8$, air cooler 201E-3 and water cooler 201E-2 (PFD 201-B-02). The cooled stream is sent to a three phase separator, $201 \mathrm{C}-6$, wherein the aqueous water stream, the liquid hydrocarbon stream, and the vapor stream are separated.

The vapor stream from $201 \mathrm{C}-6$ is cooled to $50^{\circ} \mathrm{F}$ in exchangers $201 \mathrm{E}-10$ and $201 \mathrm{E}-11$ to condense additional hydrocarbons in 201C-20. Exchanger 201E-11 is cooled by a $\mathrm{LiBr}$ solution. The liquid hydrocarbons from $201 \mathrm{C}-20$ are sent to Plant 203. The vapors (unconverted syngas) are primarily used as fuel for the gas turbine in Plant 31.

The aqueous water streams from 201C-6, 201C-9, 201C-16, and 201C-20 are sent to the waste water treatment facility. All the liquid hydrocarbon streams are sent to Plant 203.

In the absence of any specific information from STI regarding the catalyst pretreatment conditions, the design for the following catalyst pretreatment area is based on that which was developed for the previous coal Baseline F-T study. ${ }^{1}$ It is estimated that about 600 pounds per day of makeup catalyst is required to maintain the catalyst inventory in the F-T reactors. Process flow diagram PFD-201-B-03 shows the batchwise catalyst pretreatment facility which provides a continuous supply of activated, makeup catalyst. Clear wax from storage is preheated in heat exchanger 201E-5 using 600 psig saturated steam. The preheated wax is used to fill catalyst pretreater, 201C11 , and dry fresh catalyst from hopper 201T-6 is added up to the design concentration.

The system is filled with syngas and catalyst activation is carried out batchwise in $201 \mathrm{C}-11$ by circulating preheated syngas at 210 psia. The activation reactions are the reduction and carbiding of the catalyst, but some hydrocarbon product may be produced as well. The activation gas is circulated through coolers and a separator for hydrocarbon and water removal. Then it is compressed and reheated to activation temperature. Composition adjustments may be made by the addition of syngas, hydrogen and/or nitrogen. 
The activated catalyst/wax slurry is withdrawn from the bottom and sent to the slurry storage tank, 201C-12, where it is maintained hot using a hot oil circulation loop before it is pumped semi-continuously to the F-T reactors. The catalyst pretreatment section, 201-B-03, is operated for only a few hours per day to supply the makeup catalyst requirement.

\section{Material Balance}

The major streams within Plant 201 are given in Table 3.3. These stream flow rates were developed using an ASPEN Plus process flowsheet simulation model. The F-T reactor section of this model was created specifically to reproduce STI's reactor yields and generate a reactor heat balance. Furthermore, it was assumed that all of the vapor phase oxygenates remain in the vapor stream, all of the water phase oxygenates remain in the aqueous water stream, and the hydrocarbon phase oxygenates are distributed between the liquid hydrocarbon and wax streams.

\section{Technology/Licensor Selection}

The slurry F-T reactors were sized using 1) design equations provided by Syncrude Technology Inc., and 2) Bechtel in-house design and cost estimation methodology developed for previous F-T studies. ${ }^{3}$ The remainder of the design for Plant 201 was developed from in-house correlations.

The total installed cost of Plant 201 is $35.8 \mathrm{MM} \$$. This includes the cost of both STl's slurry valve (one for each F-T reactor) as well as that of the hydroclones and their underflow receiver mixers. If the hydroclones and their associated equipment are not needed, the total installed cost for Plant 201 can be reduced by $1.76 \mathrm{MM} \$$. 


\subsection{Plant 202 - Hydrogen Recovery Plant}

Plant 202, the Hydrogen Recovery Plant, provides the high purity hydrogen required by Plant 204, the Wax Hydrocracking Plant, for upgrading F-T wax product to a shippable product. The hydrogen feed stream to Plant 202 is a portion of the fresh syngas from Area 100, the Syngas Preparation Area.

\section{Design Basis and Considerations}

High purity hydrogen ( 99 mole \% H2) is produced by a standard polybed pressure swing adsorption (PSA) process from the syngas feed containing 47 mole \% hydrogen. The plant contains ten PSA adsorber vessels, packed with molecular sieves, which are cycled in sequence from 380 psig to 100 psig.

Membrane Technology (PRISM and MEDAL) were considered for cost savings. A membrane unit costs about half that of a PSA unit, but the product hydrogen has a lower purity (e.g.; 92 mole \% vs. 99 mole \% $\mathrm{H}_{2}$ ). CO and $\mathrm{CO}_{2}$ removal would be required before the recovered $\mathrm{H}_{2}$ stream could be sent to the wax hydrocracker. The potential cost savings using a membrane system would require a more detailed study including an operating cost comparison. Therefore, a standard PSA design was selected since the additional processing probably would consume most, if not all, of the cost savings.

The plant produces about 1.75 MMSCFD of hydrogen. It process about 5 MMSCFD of syngas feed (approximately $1.4 \%$ of the total syngas production).

\section{Process Description (PFD 202-B-01)}

The process flow diagram of the hydrogen recovery PSA unit is shown in Section 3.1 in PFD 202-B-01.

About $1.4 \%$ of the fresh syngas from Area 100, the Syngas Preparation Area, is split off and cooled in exchanger 202E-1 before being sent to Plant 202 for hydrogen recovery. The remaining syngas in stream 24 goes on to Fischer-Tropsch synthesis in Plant 201. The cooled syngas flows through a knock out drum, enters the feed valve, and flows upward through the adsorber vessel. As the gas flows upward through the adsorber vessel, the impurities present in the gas are selectively adsorbed. To maximize hydrogen recovery, an adsorber is switched from an adsorption position when the impurities front has reached a predetermined level in the adsorber. When an adsorber which has just been regenerated has reached adsorption pressure, it is switched to the adsorption step and the first adsorber begins its regeneration sequence.

Several concurrent blowdown steps are performed to regenerate a bed. Initially, the pressure in the adsorber is reduced to desorb hydrogen. Pure hydrogen leaving at the 
top of the adsorber is internally used in the PSA unit to repressure and purge other adsorbers. The so-called co-current or upward pressurization step is terminated when the impurities front reaches a second predetermined position in the bed. At this point, little hydrogen is left in the adsorber, and the reduced pressure has started desorption of impurities. The flow in the adsorber is then reversed, and the adsorber is depressured downward to off-gas pressure. In actuality, the regeneration process involves several pressure equalization and depressurization steps which are performed automatically.

Stream 29, the hydrogen product stream from the PSA unit is sent to Plant 204, the Wax Hydrocracking Plant.

Stream 26, the reject stream is compressed in compressor $202 \mathrm{~K}-1$ and mixed with the syngas bypassing the PSA unit in stream 24 . The combined syngas in stream 28 goes to Plant 201, the Fischer-Tropsch Synthesis Plant.

\section{TechnologyNendor Selection}

UOP's PSA polybed process is selected for this application. The unit is a standard tenbed PSA system. A bank of ten vessels filled with adsorbent, valve assemblies with piping and instrumentation and a control panel are assembled on skids.

The hydrogen recovery plant was estimated to cost $3.63 \mathrm{MM} \$$. This estimate is based on a quotation from UOP which was obtained to ensure that the low concentration of hydrogen in the syngas feed $(\sim 47$ mole $\%)$ does not present a separation problem. 


\subsection{Plant 203 - Hydrocarbon Recovery Plant}

Plant 203, the Hydrocarbon Recovery Plant, is a simplified version of that used in the coal Baseline F-T study. Because the unconverted syngas leaving the F-T synthesis plant contains so much nitrogen, it is impractical to recover as much of the light hydrocarbons for this case was done in the Baseline case. Thus, in this design, there is no refrigeration section to recover LPG and light hydrocarbons from the unconverted syngas. A LiBr chiller in Plant 201 cools the unconverted syngas to $50^{\circ} \mathrm{F}$ to recover most of the C5+ hydrocarbons before sending it to fuel. Plant 203 essentially separates the hydrocarbon liquids from Plant 201 into naphtha, distillate and wax. Some low pressure fuel gas also is produced. The product naphtha and distillate streams are sent to product blending, and the wax stream goes to Plant 204, the Wax Hydrocracking Plant, for upgrading to a shippable product.

\section{Design Basis and Considerations}

Plant 203 takes all the liquid hydrocarbon streams produced in Plant 201, the FischerTropsch Synthesis Plant, and separates them into a naphtha stream, a distillate stream, a wax stream, and a low pressure fuel gas stream. The cut points for the three liquid streams are listed below:

$\begin{array}{ll}\text { Naphtha } & \mathrm{C}_{5} \text { saturates }-350^{\circ} \mathrm{F} \\ \text { Distillate } & 350^{\circ} \mathrm{F}-650^{\circ} \mathrm{F} \\ \text { Wax } & 650+^{\circ} \mathrm{F}\end{array}$

Plant 203 essentially is a single fractionation tower with a steam side-stripper and the associated furnace, heat exchangers and flash drums.

\section{Process Description (PFD 203-B-01)}

Process flow diagram PFD 203-B-01 shows the configuration of Plant 203, the Hydrocarbons Recovery Plant.

All the liquid hydrocarbon streams from Plant 201 (streams 32, 38, 40, and 42) are combined in the product fractionator feed separator drum, 203C-10. The overhead vapor leaving this flash drum goes to the middle of the 203C-11 product fractionator to act as a stripping media. The bottoms liquids are sent to tray 9 of the 203C-11 product fractionator via pump 203G-1, exchanger 203E-14, exchanger 203E-11, and furnace $203 F-1$.

The fractionator tower separates the liquid hydrocarbons into naphtha, distillate and wax products. The fractionator tower contains 21 trays. The fractionator overhead vapor stream tower is cooled from $245^{\circ} \mathrm{F}$ to $110^{\circ} \mathrm{F}$ in air cooler $204 \mathrm{E}-12$ and water cooler 204E-13 before entering the three phase accumulator, 203C-12. The vapor 
leaving accumulator $203 \mathrm{C}-12$ is low pressure fuel gas. The condensed water stream, produced as a result of utilizing live stripping steam, is sent to the waste water treatment facility. The liquid hydrocarbon stream is refluxed back to the tower. The naphtha product stream is withdrawn from tray 2 and cooled in exchanger 203E-16. A small portion of the hot naphtha stream may be sent back to Plant 201 for use in cleaning the spent catalyst.

A 6 tray side stripper stabilizes the liquid drawn from tray 7 of the fractionator tower to provide a distillate product stream. Saturated steam at $150 \mathrm{psig}$ is used as the stripping medium. The distillate product stream is cooled by exchange with the tower feed in exchanger 203E-14 and with water in 203E-17 before going to distillate product blending.

The bottom wax stream from the product fractionator is cooled by exchange with the tower feed from $595^{\circ} \mathrm{F}$ to $350^{\circ} \mathrm{F}$ in heat exchanger 204E-11 before being sent to Plant 204, the Wax Hydrocracking Plant.

\section{TechnologyNendor Selection}

The design for Plant 203 was developed by Bechtel using open art technology. 


\subsection{Plant 204 - Wax Hydrocracking Plant}

Plant 204, the Wax Hydrocracking Plant, catalytically cracks the F-T wax product under a hydrogen environment into lower boiling materials, namely naphtha and diesel. A generic hydrocracking plant design has been selected for this study. Because of the small size of this unit, the design of the product recovery system was simplified to remove the dehexanizer column which produced a C5/C6 stream that was isomerized in the previous studies. In the current design, the C5/C6s now are part of the naphtha product. In addition, a LiBr chiller was added to cool the debutanizer overhead gas stream to $50^{\circ} \mathrm{F}$ to increase the recovery of liquid hydrocarbons from the C4- fuel gas

\section{Design Basis and Considerations}

Plant 204 takes the wax stream from the bottom of the product fractionator and cracks it is a single stage fixed-bed hydrocracker with minimum coke formation to yield higher quality naphtha and distillate. Hydrocracking occurs at about $700^{\circ} \mathrm{F}$ and between 1100 and 1500 psig under a hydrogen atmosphere in a single multi-bed reactor with inter-bed cooling by hydrogen-rich recycle gas. The reactor effluent is cooled and separated into C4- fuel gas, naphtha and distillate. Unconverted material from the bottom of the fractionator is recycled back to the hydrocracking reactor. A LiBr chiller maximizes hydrocarbon recovery from the $\mathrm{C} 4$ - fuel gas.

\section{Process Description (PFD 204-B-01)}

Process flow diagram PFD 204-B-01 shows the configuration of Plant 204, the Wax hydrocracking Plant.

Stream 47, the wax stream from the Hydrocarbons Recovery Plant, is mixed with unconverted material from the bottoms of the $204 \mathrm{C}-8$ product fractionator and hydrogen rich gas before being heated in exchanger 204E-1 and furnace 204F-1. The heated reaction mixture enters the top of the multi-bed, downflow hydrocracking reactor. For simplicity, only two beds are shown in PFD 204-B-01. Interbed cooling is obtained by injection of hydrogen-rich gas. The reactor effluent is cooled to $110^{\circ} \mathrm{F}$ in exchangers 204E-1 and 204E-3 and air cooler 204E-2 before being flashed in 204C-1. The hydrogen-rich gas leaving the 204C-1 flash drum is compressed in 204K-3, heated and recycled back to the hydrocracking reactor.

The liquid leaving the $204 \mathrm{C}-1$ flash drum is sent to the $204 \mathrm{C}-4$ debutanizer column which produces and overhead vapor stream and a liquid hydrocarbon stream. The overhead vapor stream is mixed with low pressure fuel gas streams from Plants 201 and 203 (streams 37 and 44, respectively) and low pressure gas from the 204C-8 product fractionator and sent to the $204 \mathrm{E}-11 \mathrm{LiBr}$ chiller to maximize liquid hydrocarbon recovery. The chiller effluent is flash in the $204 \mathrm{C}-11$ flash drum; the overhead of which goes to fuel gas, and the bottoms is mixed with the debutanizer bottoms stream and sent to the $204 \mathrm{C}-8$ product fractionator after passing through the 204F-2 furnace. The 
fractionator overhead product stream is the naphtha product, stream 50 . Stream 51 , the distillate product is withdrawn from the middle of the product fractionator through the 204C-10 side stream stripping column. The bottoms from the product fractionator is mixed with fresh feed and recycled back to the hydrocracking reactor.

\section{TechnologyNendor Selection}

The design for Plant 204 was developed by Bechtel using open art technology. Several vendors will license hydrocracking technology. Chevron and UOP are two of the major licensors of hydrocracking processes. 


\section{COMBINED-CYCLE POWER PLANT AND OFFSITES PLANTS}

In a conventional plant scenario, the power plant is considered an offsites plant. However, in this situation, the combined-cycle power plant is an important component of the system and is considered to be an ISBL plant. Therefore, it is discussed in somewhat detail in Section 6.1.

Section 6.2 provides a brief overview of the other offsites plants. These plants are discussed in more detail in the 1994 topical report describing the process design for the coal Baseline F-T study. ${ }^{1}$ As expected, some modifications and revisions were made to these offsites plants because the feedstock is natural gas and not coal. These revisions are discussed in Section 6.2 along with a brief description of each offsites plant. 


\subsection{Plant 31 - Combined-Cycle Power Plant}

Plant 31, the Combined-Cycle Power Plant, consumes the C4- fuel gas produced in the F-T plant as well as the excess 635 psig steam from Plant 102 and the excess 150 psig stream from Plant 201 to produce electric power, compress the inlet air to Plant 101 to 665 psia, and produce 50 psig steam for use within the process area. The fuel gas is burned in a General Electric (GE) Frame 7 gas turbine which drives the air compressors and an electric power generator. The hot exhaust gases from the air compressor and the inlet steams from Plant 102 and 201 go to the HRSG (Heat Recovery Steam Generation) section where three levels of superheated steam are generated and sent to the three-stage steam turbine which drives another power generator. 50 psig steam is withdrawn from the low pressure turbine for use in other parts of the plant.

Plant 31 supplies the complete electric power needs for the entire complex as well as producing an extra $84 \mathrm{MW}$ for sale.

\section{Design Basis and Considerations}

A combined-cycle power plant with an integrated HRSG that is matched to the steam levels produced by and/or consumed by the process plants was selected because it is . the most efficient means of power generation. A General Electric (GE) Frame 7 gas turbine is used to drive the air compressors and a power generation turbine. The HRSG section was simulated using the GateCycle computer simulation program to determine the steam productions and power production by the three-stage steam turbines. ${ }^{4}$

\section{Process Description (PFD 31-B-01)}

A simplified schematic diagram of Plant 31, the Combined-Cycle Power Plant, is given in PFD 31-B-01. The fuel gas from Plants 201, 203 and 204 along with some steam for $\mathrm{NO}_{x}$ control are burned in the GE Frame 7 turbine. A multi stage air compressor and a power generation turbine are directly driven by the gas turbine. The air compressor provides 665 psia inlet air for Plant 101 as well as the lower pressure air that is burned in the combustion section of the turbine. The power generation turbine produces 90.7 MW of electric power.

Recoverable heat from the gas turbine exhaust is transferred to the steam power cycle in the HRSG. The HRSG has a series of fined-tube heat exchanger tube banks, installed in an insulated casing. The gas turbine exhaust flows successively through the superheater, boiler and economizer sections producing high pressure (HP), intermediate pressure (IP), and low pressure (LP) superheated steams. The HP steam is 975 psig. The IP steam is 635 psig, and the LP steam is 50 psig. The 635 and 50 psig steam levels were set by the production and demand requirements of the other ISBL plants. 
About 316 thousand pounds per hour of $700^{\circ} \mathrm{F}$ superheated 635 psig steam is produced in the steam superheater (not shown in PFD 31-B-01) from the saturated 635 psig steam produced in Plant 102 that is not consumed by the other ISBL plants. About 155 thousand pounds per hour of 50 psig steam at $365^{\circ} \mathrm{F}$ are produced from the 150 psig steam generated in the HRSG by withdrawal from the low pressure section of the steam turbine. The power generator driven by the steam turbine produces $92.6 \mathrm{MW}$ of electric power.

Thus, the total power production by Plant 31 is $183.3 \mathrm{MW}$.

Technology Nendor Selection

A general Electric Frame 7 gas turbine was selected for the combustion turbine. There are several suppliers for the HRSG; some of which are Deltak, Nooter/Eriksen, Vogt, and Zurn. 


\subsection{Offsites Plants}

Detailed process descriptions of the offsites plants are not presented. They have been previously reported in the 1994 topical report describing the process design for the coal Baseline F-T study. ${ }^{1}$ Except for the combined-cycle power plant, which is described in the previous section, the offsites plant requirements were prorated from the coal Baseline F-T study. However, Plant 24, the Coal Ash Disposal Plant, is no longer applicable and has been deleted.

Following is a brief description of each offsites plant with comments noting any departure in the design of it from the coal Baseline F-T case.

Plant 19 - Relief and Blowdown - Plant 19 is for the collection and flaring of relief and blowdown discharges from all applicable plants. It includes two flare systems; a main flare for all hydrocarbon containing discharges and a small secondary flare for emissions containing $\mathrm{H}_{2} \mathrm{~S}$. Collection piping is not included in Plant 19 but has been included in Plant 21, Interconnecting Piping.

Plant 20 - Tankage - Plant 20 provides storage and delivery equipment for products, intermediates and chemicals. Thirty days storage is provided for the naphtha, distillate and butanes products. Two days intermediate storage is provided for the feed to Plant 204, the Wax Hydrocraking Plant. This two day storage capacity is required to provide feedstock during plant startup and to mitigate the effect on operations due to brief interruptions in the upstream plants which could be the result of scheduled or unscheduled maintenance or due to operating problems.

Since byproduct sulfur is no longer produced, no sulfur facilities are provided.

Plant 21 - Interconnecting Piping System - Plant 21 includes the interconnecting process and utility piping between process plants and offsites. All above ground and underground piping systems are included except the cooling water piping which is included in Plant 32, Cooling Water Distribution, and the fire water piping which is included in Plant 33, Fire Systems. Relief and blowdown headers are included. In general, water distribution piping is underground and all other piping is located above ground on pipe racks. Storm sewers, sanitary sewer and process wastewater lines are not part of this plant but are included in Plant 34, Sewers and Wastewater Treating.

Plant 22 - Product Shipping - Plant 22 provides the pipeline, pumping and metering systems for delivery of the final hydrocarbon products. Separate systems are provided for each of the hydrocarbon products. Dual meters are provided to assure proper recording and product delivery.

Plant 23 - Tank Car/ Tank Truck Loading -- Plant 23 provides the tank car and truck loading racks for the hydrocarbon products. Catalyst unloading blowers also are 
provided to transfer the catalyst from the road receiving point to the storage silo in Plant 201.

Since byproduct sulfur is no longer a produced, no sulfur loading facilities are provided.

Plant 25 - Catalyst and Chemical Handling - Plant 25 provides storage and handling for the catalyst and chemicals used in all the plants. Additionally, it provides a consolidated location for tracking catalyst and chemical start-up and daily consumption requirements. This plant includes an enclosed warehouse for storage and forklifts for transporting pallets into or out of the warehouse.

Plant 30 - Electrical Distribution System - Plant 30 provides the electrical distribution system from the $115 \mathrm{kV}$ high voltage switchyard to the consuming locations.

Plant 32 - Raw, Cooling and Potable Water - Plant 32 takes raw water from a nearby lake or river and provides raw water treatment consisting of

- Water clarification

- Gravity filtration

- Potable water chlorination

- Demineralization

The clarified water is used for cooling tower makeup, fire fighting and utilities. A packaged potable water system is used to treat the water used for drinking, food preparation and sanitary facilities. Clarified and filtered water is the feed to this packaged potable water system. The boiler feed water is clarified, filtered and demineralized.

Plant 33 - Fire Protection System - The fire protection and control systems for all facilities, structures and equipment within the plant area are designed in compliance with federal, state and local jurisprudence codes and standards, and with the recommendations of the American Petroleum Institute, National Fire Protection Association, Industrial Risk Insurer, and the Oil AAA Insurance Association.

A comprehensive fire water system is provided for general fire protection of the entire plant. Chemical and steam fire suppression systems are provided for specific facilities and equipment. These systems include

- $\quad$ Fire water to process plants, water and waste treatment, and tankage

- $\quad$ Fireproofing for vessel supports, pipe racks, etc.

- $\quad$ Sprinkler systems for buildings, parts of the process equipment such as pumps or heat exchangers (depending on the location), and tank truck and tank car filling racks.

- Smothering steam for compressor buildings and fired heaters

- Halogen system for computer room and laboratory 
Plant 34 - Sewage and Effluent Water Treatment - Plant 34 provides segregated waste water treatment for the purpose of minimizing both raw water consumption and effluent discharge to public waters during normal plant operation. Waste water streams are segregated on the basis of their compatibility and treated as necessary to make them suitable for reuse, if practical, in lieu of fresh water.

The majority of the water used in the project eventually goes to the atmosphere as water vapor. Some water is disposed of as moisture associated with solid wastes going to landfills. Blowdown streams (cooling tower, boilers and demineralizer) are sent to an intermediate holding pond before being discharged.

Plant 34 contains the following treatment facilities

- Oily wastewater treatment

- $\quad$ Process wastewater treatment

- $\quad$ Solids dewatering

- Sanitary sewage treatment

Since this facility does not process any coal, the coal pile storage runoff treatment facilities are not required and have been removed.

Plant 35 - Instrument and Plant Air Facilities - Plant 35 includes all equipment necessary to supply instrument and utility air to the process plants and support facilities. The distribution piping is included in Plant 21, Interconnecting Piping. Instrument and utility air is dry, oil-free and dirt-free air that is supplied at $100 \mathrm{psig}$. It has a maximum dew point of $-40^{\circ} \mathrm{F}$.

Plant 36 - Purge and Flush Oil System - Plant 36 provides and delivers a light and heavy flush oil for pump seal flushing and instrument purging.

Plant 37 - Solid Waste Management - Plant 37 disposes of wastes from Plant 32 (Raw, Cooling and Potable Water), Plant 34 (Wastewater Treatment), and miscellaneous sources which include refuse and flotsam.

All the solid waste, excluding the miscellaneous plant refuse, is stored in bins and hoppers, and collected daily to minimize on-site storage. Once collected, it is transported to an approved landfill disposal site outside the battery limits in trucks.

Plant 40 - General Site Preparation - Although this plant is located at a generic Gulf Coast site, the general site preparation costs were prorated from those used for the coal Baseline F-T design. ${ }^{1}$ That plant is located in Southern Illinois at a green field location with rail and road accessibility. Site preparation involves leveling the land and adding basic improvements such as roads, fencing and drainage needed by the plant as a whole, and the placement of high load-bearing fills, pilings, spread footings and mat foundations for the plant structures in accordance with individual needs. 
Drainage of contaminated rain runoff from process and offsite areas is directed to ponds for treatment. Uncontaminated storm runoff from building roofs, parking lots, outdoor storage areas, and uncontaminated process plant areas is routed to the raw water storage pond.

Plant 41 - Buildings - Five different types of buildings are provided for different . usages. The type of construction selected for each building is dependent on its location with respect to potential hazards, its criticality for plant operation, and its function. The five types of buildings are classified as types A, B, C, D or Administrative according to the major construction features. Type $A$ buildings are blast-proof and house critical equipment and/or instrumentation for the continuous operation of the plant. Type B buildings house the plant laboratory, cafeteria, medical building and change house. Type $C$ buildings are steel-framed structures which serve a number of diverse functions which are generally plant operations or maintenance related. Type D buildings have masonry walls and structural steel-framed roofs and are used for transformer shelters and chemical storage. The administration building (which also contains the computer room) is identical in construction to a Type B building except that the exterior is finished with brick veneer masonry.

Plant 42 - Telecommunications System - Plant 42 includes the equipment and wiring for communication throughout the plant, to offsite locations linking plant data processing systems with offsite computing facilities, and for communication with transportation carriers. Plant 42 provides

- Interconnecting cables, standby emergency power and grounding

- Remote computer access

- Facsimile

- Fire alarm

- Public address paging

- Medical emergency and life-signs telemetry

- Interplant part paging

- Land mobile radio

- Radio paging

- Security system

- Telephone, telephone PABX

Plant 43 - Distributed Control System and Software - Plant 43 provides for the distributed control system and operator interface in one central control system except for the shipping and loading facilities which are located at the shipping and loading building. 


\section{OVERALL CATALYST AND CHEMICAL REQUIREMENTS}

The overall catalyst and chemicals requirements for this once-through, natural gas based F-T plant are shown in Table 7.1. They are broken down according to process areas. This table shows both the initial requirements as well as the estimated annual consumptions.

\section{Table 7.1}

Overall Catalyst and Chemical Requirements

\begin{tabular}{|c|c|c|c|}
\hline Catalyst & Plant & $\begin{array}{c}\text { Initial } \\
\text { Requirement }\end{array}$ & $\begin{array}{c}\text { Annual } \\
\text { Consumption }\end{array}$ \\
\hline \multicolumn{4}{|l|}{ Area 100} \\
\hline Sulfur Polishing (ZnO) & 102 & $6,130 \mathrm{lb}$ & $1,250 \mathrm{lb} / \mathrm{yr}$ \\
\hline MDEA for $\mathrm{CO}_{2}$ Removal & 103 & $45,300 \mathrm{lb}$ & $11,330 \mathrm{lb} / \mathrm{yr}$ \\
\hline \multicolumn{4}{|l|}{ Area 200} \\
\hline FT Cobalt Catalyst & 201 & $242,000 \mathrm{lb}$ & $193,000 \mathrm{lb} / \mathrm{yr}$ \\
\hline UOP LPHC Catalyst & 204 & $28,300 \mathrm{lb}$ & $4,040 \mathrm{lb} / \mathrm{yr}$ \\
\hline \multicolumn{4}{|l|}{ Offsites } \\
\hline $\begin{array}{l}\text { Alum* } \\
\text { Polymer } \\
98 \% \mathrm{H}^{*} \mathrm{SO}^{*} \\
50 \% \mathrm{NaOH}^{*}\end{array}$ & 32 & $\begin{array}{l}9,460 \mathrm{lb} \\
2,840 \mathrm{lb} \\
4,730 \mathrm{lb} \\
9,460 \mathrm{lb}\end{array}$ & $\begin{array}{r}1,480 \mathrm{lb} / \mathrm{yr} \\
560 \mathrm{lb} / \mathrm{yr} \\
4,950 \mathrm{lb} / \mathrm{yr} \\
1,220 \mathrm{lb} / \mathrm{yr}\end{array}$ \\
\hline $\begin{array}{l}\text { Chlorine } \\
\text { Polymer } \\
\text { PAC }\end{array}$ & 34 & $\begin{array}{r}570 \mathrm{lb} \\
850 \mathrm{lb} \\
1,710 \mathrm{ft}^{3}\end{array}$ & $\begin{array}{l}100 \mathrm{lb} / \mathrm{yr} \\
130 \mathrm{lb} / \mathrm{yr} \\
570 \mathrm{ft}^{3} / \mathrm{yr}\end{array}$ \\
\hline
\end{tabular}




\section{OVERALL LABOR REQUIREMENTS}

In contrast to the coal Baseline F-T design study, a complete labor and staffing plan was not developed. Direct operating labor for each of the ISBL plants was estimated, and the number of extra operators, foremen and maintenance workers was obtained by ratio to the direct operating labor.

The overall estimated labor requirements for this plant are shown in the following table.

\section{Table 8.1}

Overall Labor Requirements

Plant Description

101 Air Separation Plant

102 Autothermal Reforming Plant

$103 \mathrm{CO}_{2}$ Removal and Recycle Plant

201 Fischer-Tropsch Synthesis Plant

202

203

204 Hydrogen Recovery Plant Hydrocarbon Recovery Plant Wax Hydrocracking Plant

Number of

Personnel

Total Dedicated Plant Operators

20

Extra Operators, Foremen and Maintenance Workers

Total Labor Requirement 60 


\section{PLANT COSTS}

\subsection{Installed Plant Costs}

Table 9.1 shows the costs for this once-through, natural gas F-T liquefaction plant. A few of the ISBL plant costs were developed by adjustment and capacity proration from the previous coal Baseline F-T study and the natural gas Fischer-Tropsch liquefaction case. $^{1,2}$

\section{Table 9.1}

Cost Breakdown of the Once-Through F-T Liquefaction Plant

\begin{tabular}{|c|c|c|c|}
\hline Plant & Description & Cost (MM\$) & $\%$ ISBL \\
\hline 101 & Air Compression \& Separation & 70.4 & 32.7 \\
\hline 102 & Autothermal Reforming & 22.8 & 10.6 \\
\hline 103 & $\mathrm{CO}_{2}$ Removal and Recycle & 13.4 & 6.2 \\
\hline 201 & Fischer-Tropsch Synthesis & 35.8 & 16.6 \\
\hline 202 & Hydrogen Recovery & 3.6 & 1.7 \\
\hline 203 & Product Fractionation & 3.2 & 1.5 \\
\hline 204 & Wax Hydrocracking & 11.8 & 5.5 \\
\hline 31 & Combined Cycle Plant & 54.5 & 25.3 \\
\hline & Total ISBL & 215.5 & \\
\hline & Offsites Facilities & 120.3 & \\
\hline & Subtotal: & 335.8 & \\
\hline & HO Service/Fees/Contingency & 79.4 & \\
\hline & Total Installed Cost: & 415.2 & \\
\hline
\end{tabular}




\subsection{Annual Catalyst and Chemical Costs}

Table 9.2 shows the various annual catalyst and chemical costs for this once-through natural gas liquefaction plant. These costs are estimated based on 1996 prices.

\section{Table 9.2}

Annual Catalyst and Chemical Costs for the Once-Through F-T Liquefaction Plant

\begin{tabular}{rllrr} 
Plant & Description & Material & Cost, MMS \\
\cline { 1 - 1 } 102 & Autothermal Reforming & ZnO & 261,400 \\
& & Catalyst & 876,000 \\
103 & $\mathrm{CO}_{2}$ Removal and Recycle & MDEA & 17,000 \\
201 & Fischer-Tropsch Synthesis & Catalyst & $3,630,000$ \\
204 & Wax Hydrocracking & Catalyst & 40,400 \\
32 & Raw, Cooling and Potable Water & All 5 & $1,120,000$ \\
34 & Sewage and Effluent Water Treatment & All 3 & 102,700 \\
& Total & & $6,047,500$
\end{tabular}




\section{SUMMARY AND RECOMMENDATIONS}

A conceptual design for a once-through natural gas, Fischer-Tropsch (F-T) plant with power co-production has been developed to examine the economic viability of the conversion of natural gas to syncrude and/or liquid transportation fuels. This design incorporates an enriched air blown autothermal reformer for syngas generation and a proprietary catalytic process for F-T synthesis from Syncrude Technology, incorporated (STI). In developing this once-through F-T conceptual design, where applicable, individual plant designs and cost estimates were prorated directly from previous F-T indirect coal liquefaction and natural gas studies.

The plant processes $100 \mathrm{MMSCF} /$ day of natural gas and produces about $8815 \mathrm{BPD}$ of F-T liquid products. These liquid products consist of 146 bbl/day of butanes, 2933 bbl/day of a $\mathrm{C} 5-350^{\circ} \mathrm{F}$ naphtha, and $5736 \mathrm{bbl} /$ day of a $350-850^{\circ} \mathrm{F}$ distillate. The naphtha and distillate are raw products. Both are free of sulfur, nitrogen and oxygen containing compounds and can be further processed to produce premium gasoline and diesel blending stocks. The plant uses all of the byproduct steam and fuel gas production to supply its electric power requirements. In addition, it produces about 84.1 MW of excess electric power. The only raw materials delivered to the plant are natural gas, water, catalysts and chemicals.

The total capital cost for the plant is estimated at $415 \mathrm{MM}$ mid-1996 dollars when constructed at a generic U. S. Gulf Coast location.

There are significant differences between the present once-through F-T design with that of the previous natural gas F-T study which was for a plant that is over four times larger. This once-through F-T power co-production design was developed to reduce capital cost and does not contain any naphtha or distillate product upgrading steps. Wax hydrocracking facilities are included to reduce the boiling range of the raw F-T liquids. When compared at a same natural gas feed rate of $100 \mathrm{MMSCFD}$, this once through design costs about $32 \%$ less than that of the previous natural gas recycle case. This reduction in capital cost is achieved at a slight sacrifice in the overall process thermal efficiency.

A preliminary economic analysis was carried out comparing this once-through F-T power co-production case with the previous maximum liquid production design using gas-recycle. Using the same methodology as was used in the previous studies (a 0.50 \$/MMBtu gas price, 1996 ElA escalation factors, and treating the F-T product as a highvalue syncrude having a premium above that of conventional crude), the original F-T gas-recycle design has a COE (crude oil equivalent) price of $19.1 \$ / \mathrm{bbl}$. When this design is capacity-factored down from 412 MMSCFD to 100 MMSCFD, the COE increases to about $23.0 \$ / \mathrm{bbl}$ - approximately a $20 \%$ increase due solely to the loss in the economy of scale. 
For the once-through, power co-production design, 84.1 MW of excess power is produced. Assuming an electricity selling price of 3 cents per KWh to a IPP (independent power producer), this case has a COE of $20.6 \$ / \mathrm{bb} /$ with a syncrude premium factor of $10 \%$ (i.e., the combined F-T naphtha and distillate products have a value that is $10 \%$ higher than the price of petroleum crude). This represents a $12 \%$ decrease in the COE compared to the gas-recycle case.

The reduction in COE increases to $22 \%$ if a syncrude premium factor of $20 \%$ is assumed. It is not unreasonable to believe that F-T naphtha and distillate can command such a premium since, in effect, the F-T product is equivalent to a bottomless, nitrogen-free and sulfur-free crude oil. Furthermore, under the right conditions, the benefit of power co-production can be significant. This advantage could be what is needed to allow smaller once-through, Fischer-Tropsch gas liquefaction plants to be economically viable.

Site-specific studies are recommended to identify further economic improvements. For example, the following conditions could result in additional economic benefits.

1. Using associated gas or low-quality natural gas containing 10 to $12 \% \mathrm{CO}_{2}$

2. Using an air-blow, instead of an enriched air-blow, gasification process

3. Potable water production

4. Chemical co-production

5. Other site-specific symbiosis effects

6. Removal of the wax hydrocracker and shipping the raw combined F-T liquids either separately and/or mixed with conventional crude to a refinery for upgrading. 


\section{REFERENCE}

1. Topical Report, Volume I, Process Design - Illinois No. 6 Coal Case with Conventional Refining, Baseline Design/Economics for Advanced Fischer-Tropsch technology, DOE Contract No. DE-AC22-91PC90027, October, 1994.

2. Topical Report VI, Volume I, Natural Gas Fischer-Tropsch Case, Volume I, Summary Report and Volume II, Plant Design and Aspen Process Simulation Model, DOE Contract No. DE-AC22-91PC90027, August, 1996.

3. Final Report, Slurry Reactor Design Studies - Slurry vs. Fixed-Bed Reactors for FischerTropsch and Methanol, DOE Contract No. DE-AC22-89PC89867, June, 1990.

4. GateCycle computer simulation program, Power Cycle Simulation Software, ENTER Software Company, Menlo Park, CA. 


\section{APPENDIX}

Presentation at 1997 Coal Liquefaction and Solid Fuels Contractors Review Conference 
Design/Economics of a Once-Through Natural Gas Fischer-Tropsch Plant With Power Co-Production

Gerald N. Choi, Sheldon J. Kramer, Samuel S. Tam

(Bechtel Corporation)

Joe M. Fox

(Consultant)

Norman L. Carr, Geoffrey R. Wilson

(Syncrude Technology, Inc.)

1997 Coal Liquefaction \& Solid Fuels

Contractors Review Conference

September 3-4, 1997

Pittsburgh, Pennsylvania 


\title{
Design/Economics of a Once-Through Natural Gas Fischer-Tropsch Plant With Power Co-Production
}

\author{
Gerald N. Choi. Sheldon J. Kramer. Samuel S. Tam \\ (Bechtel Corporation) \\ Joe M. Fox \\ (Consultant) \\ Norman L. Carr, Geoffrey R. Wilson \\ (Syncrude Technology. Inc.)
}

\section{INTRODUCTION}

In 1994, Bechtel, along with Amoco as the main subcontractor, developed a Baseline Design (and an ASPEN Plus computer process simulation model) for indirect coal liquefaction using advanced Fischer-Tropsch (F-T) technology to produce highquality, liquid transportation fueis. This was done under DOE Contract No. DE-AC22-91 PC90027. In 1995, the original study was extended to develop a case in which natural gas, instead of coal. is used as the feedstock.' The results, presented at last year's Contractors' Conference, show that a natural gas F-T plant is less capital intensive. and as a consequence. attractive F-T economics may be attained with low cost remote gas."

In 1996, the natural gas based F-T study was extended to develop a conceptual design for a once-through F-T plant with power co-production using remote gas as the feedstock. This once-through facility is designed to reduce the initial cost at the expense of a small sacrifice in overall thermal efficiency. In addition. the product upgrading area is simplified to produce only shipable F-T liquids. This paper describes the results of this study. It discusses the overall plant design, preliminary economics, and some site-specific situations under which additional capital cost savings can be realized.

\section{DESIGN BASIS}

The plant is designed to process $100 \mathrm{MMSCF} /$ day of natural gas and produce roughly $8,820 \mathrm{BPD}$ of F-T syncrude and 84 MW of export power. For design purposes, a typical natural gas composition containing about 95 mole\% methane was assumed. The F-T liquefaction plant design is based on the Syncrude Technology Incorporated (STI) cobalt-based, F-T catalyst. In this design, the $\mathrm{H}_{2} / \mathrm{CO}$ ratio and the partial pressures of $\mathrm{H}_{2}$ and $\mathrm{CO}$ in the syngas are important for proper F-T synthesis reactor performance. An autothermal reformer using enriched air with partial $\mathrm{CO}_{2}$ recycle is used for syngas preparation. This design was arrived at after studying several alternatives, including air-blown autothermal reforming operating at low steam/carbon feed ratio. After satisfying the internal fuel and heating requirements. the plant sends all of the byproduct steam and tail gas to a combined-cycle plant for air compression and power generation.

The plant is assumed to be located at a hypothetical U. S. Gulf Coast site, a common basis for such studies. For guidance as to how to relate such costs to remote sites. see the earlier natural gas based study ${ }^{2}$. In developing this case. individual plant designs and cost estimates were prorated, where applicable. from the previous coal and natural gas based deisgns ${ }^{1.2}$.

\section{OVERALL PLANT CONFIGURATION}

\section{Svngas Generation and F-T Sunthesis}

Figure 1 is a simplified block flow diagram showing the process configuration of the synthesis section. This portion of the plant consists of two main processing areas: Area 100, synthesis gas preparation, and Area 200, once-through F-T synthesis and product fractionation. This porion of the plant was simulated using ASPEN Plus. In the F-T synthesis plant, the ASPEN Plus model was modified to duplicate the results of STI's reactor model.

\section{Combined Cycle Power Plant}

Figure 2 is a simplified block flow diagram showing the combined-cycle plant which uses a General Electric (GE) Frame 7 gas turbine. In addition to producing power. this gas turbine drives the air compressors for the air separation plant. The 635 
psig steam from the ATR and the 150 psig steam from the F-T synthesis plant are superheated and imported directly into the steam turbines. Overall performance of the combined-cycle plant was calculated using the GateCycle power cycle simulation software (Enter Software, Menlo Park. CA).

Outside Batter Limits (OSBL) Plants

There are eighteen ancillary offsite plants. ranging from water treatment to interconnecting piping, which are similar to those which were developed for the coal-based Baseline Design and were modified. as required. for this natural gas case. ${ }^{3}$

\section{SYNTHESIS GAS PREPARATION (AREA 100)}

This area consists of three major plants; air compression and separation, autothermal reforming (ATR), and $\mathrm{CO}_{2}$ removal and recycle. The objective of Area 100 is to produce a nitrogen-diluted syngas with a molar $\mathrm{H}_{2} / \mathrm{CO}$ ratio of 2.0 . Sulfur is removed from the natural gas before syngas generation by adsorption on $\mathrm{ZnO}$. The $\mathrm{H}_{2}$ and $\mathrm{CO}$ partial pressures entering the F-T slurry-bed reactors also were governing factors in the design of the syngas preparation area. Autothermal reforming calculations/trade-off studies were carried out to determine that the syngas requirements could best be met with an ATR using $40 \%$ enriched air operating with $\mathrm{CO}_{2}$ recycle. The ATR reactor is designed to operate at a reasonable 1 ) $\mathrm{O}_{2} / \mathrm{C}$ ratio of 0.7 to keep the maximum adiabatic flame temperature below $4000^{\circ} \mathrm{F}$, and 2) stearn/C ratio of 0.6 to avoid potential soot formation. The final syngas preparation design was confirmed by Lurgi Corporation. ${ }^{4}$

Plant 101. the air compression and separation plant. supplies enriched air containing 40 mole\% oxygen at 650 psig to the ATR The 95 mole\% $\mathrm{O}_{2}$ product from cryogenic air separation is diluted with bypassed, compressed air to produce the required enriched air feed.

\section{FISCHER-TROPSCH SYNTHESIS AND PRODUCTION FRACTIONATION (AREA 200)}

This area consists of four plants; once-through F-T synthesis, product separation, hydrogen recovery and wax hydrocracking. The wax hydrocracking design is similar to that used in the previous F-T studies. Its objective is to ensure that the produced F-T product is pumpable at ambient conditions. A small hydrogen recovery (PSA) plant is used to recover enough hydrogen for hydrocracking. The PSA plant feed is a portion of the syngas leaving Area 100 rather than the unconverted syngas from the F-T synthesis plant, as was done in the previous recycle designs, because the hydrogen concentration in the F-T effluent is too dilute for effective hydrogen recovery. The effluent from the PSA plant goes to the F-T synthesis reactors.

A total of three, 6 meter diameter by 20 meter high, slurry-bed reactors are required to process the syngas from Area 100. These reactors are arranged so that two parallel first-stage reactors feed a single second-stage reactor. The unconverted syngas leaving the first-stage is cooled to $150{ }^{\circ} \mathrm{F}$ and flashed to condense and remove liquids before being reheated and fed to the second-stage. The $\mathrm{CO}$ conversion in each of the $\mathrm{F}-\mathrm{T}$ reactors is about $61 \%$ with an overall $\mathrm{CO}$ conversion of about $85 \%$. The first-stage F-T slurry reactors operate at about $430{ }^{\circ} \mathrm{F}$ and $490 \mathrm{psig}$, and the second-stage reactor operates at about $430^{\circ} \mathrm{F}$ and $440 \mathrm{psig}$. Excess heat is removed by the generation of 150 psig steam from tubes within the reactors.

The design is based on STI's specified F-T kinetics and product distribution. The slurry-bed reactors are sized in accordance with STI's guidelines and include STI's proprietary catalyst/wax separation technology. Overall reactor weight and cost were determined using Bechtel's slurry-bed reactor design model. ${ }^{\text {s }}$

The hydrocarbon product recovery section consists of a conventional fractionation column with a side stripper to recover a naphtha stream, a distillate stream and a wax stream which goes to the wax hydrocracking plant. The column overhead stream goes to a $\mathrm{LiBr}$ absorption chiller, using waste heat, to recover additional hydrocarbons. Plant 204 , the wax hydrocracking plant, is similar to that of the previous cases except that the fractionation system has been simplified to produce only butanes, naphtha and distillate products. The light gases also go to the LiBr cooled chiller to maximize hydrocarbon recovery. 


\section{PLANT SUMMARY}

\section{Process Design Basis}

The plant processes $100 \mathrm{MMSCF} /$ day of natural gas and produces about $8.820 \mathrm{BPD}$ of liquid F-T products; namely a $\mathrm{C}_{5}-350^{\circ} \mathrm{F}$ naphtha, a $350-850^{\circ} \mathrm{F}$ distillate. and a small amount of butanes. Both the naphtha and distillate are essentially free of sulfur and nitrogen containing compounds. The naphtha is a raw product which either can be upgraded to produce a high-quality gasoline blending stock or used as a feedstock for steam cracking to produce light olefins. The distillate is a high-quality diesel blending stock which requires no further upgrading. The plant uses all the byproduct steam and fuel gas to supply its internal electric power and heating requirements. In addition. it produces about $8+\mathrm{MW}$ of exported electric power. The only materials delivered to the plant are natural gas, raw water. catalysts and chemicals. The major feed and product streams entering and leaving this plant are shown in Table 1.

Following the philosophy of the indirect coal liquefaction study. the overall plant is designed to comply with all applicable environmental, safety and health regulations. Air cooling is maximized, wherever possible, in order to minimize cooling water usage.

\section{Capital Cost Estimate}

Total capital cost of this natural gas F-T plant is about \$+15 MM (mid 1996 dollars). This cost includes offsites and allowances for home office costs, service fees and contingency. Since the exported powver is a major product, the combinedcycle plant is considered to be one of the ISBL plants. Table 2 shows a breakdown of the capital cost. Area 100. the syngas preparation area, is about half of the total ISBL cost with the remaining half being equally divided between Area 200, the F-T synthesis and product recovery area, and Plant 31 , the combined-cycle power plant. The air compression and separation (Plant 101) and combined-cycle (Plant 31) plants are the two most expensive ISBL plants. and together. they constitute 58\% of the ISBL cost. The estimated cost of this once-through F-T plant is about a third less than that of a F-T plant of the same size using gas recycle to maximize liquid production.

\section{ECONOMIC SENSITIVITY STUDIES (PRELIMINARY)}

A discounted-cash-flow analysis on the production cost of the F-T products was carried out 1 ) to compare this once-through, F-T power co-production design with that of the previous natural gas design which uses gas recycle for maximum liquid production. and 2) to examine the economics of this once-through case for remote gas using the same methodology and financial assumptions employed in the F-T baseline study. ${ }^{2}$ As in the previous studies, the results are expressed in terms of a crude oil equivalent (COE) price which is defined as the hypothetical break-even crude oil price where the F-T liquefaction prodicts are competitive with products made from crude oil.

The primary liquefaction products consist of about $1 / 3$ raw naphtha and $2 / 3$ distillate. The distllate is a high-quality diesel blending stock which requires no further upgrading. A past linear programming study of a typical PADD II refinery indicated the F-T distillate can command a premium of $7.19 \mathrm{~S} / \mathrm{bbl}$ above and beyond the value of conventional crude oil. ${ }^{6}$ For the following analysis, it was assumed that the raw naphtha has the same value as that of crude oil.

The comparison between this once-through power co-production design with the previous gas-recycle case is carried out using 0.50 S/MMBtu gas, estimated 1993 costs and 1996 EIA escalation factors. Figure 3 shows the results in terms of COE vs. the electricity selling price. The original recycle plant design processes about $412 \mathrm{MMSCF} /$ day of gas feed and has a COE price of $19.1 \mathrm{~S} / \mathrm{bbl}{ }^{1.2}$ The COE has only a slight dependence on the electricity selling price since the plant exports only a small amount of power. When the plant cost is capacity-factored to process $100 \mathrm{MMSCF} /$ day of gas feed. the COE increases to about $23.0 \mathrm{~S} / \mathrm{bbl}$ - approximately a $20 \%$ increase just due to the loss in the economy of scale.

The once-through F-T power co-production plant produces $84 \mathrm{MW}$ of excess power for sale. and Figure 3 shows that its economics are very sensitive to the selling price of the co-produced power. Typical base load power plants sell electricity between + to 6 cents per $\mathrm{kWh}$. Even at a conservative power selling price of 3 cents per $\mathrm{kWh}$. the plant has a COE price of $18.8 \mathrm{~S} / \mathrm{bbl}$. This COE is $22 \%$ less than that of the scaled down recycle gas case which maximizes liquids production. 
Figure 4 shows the porion of the calculated COE price attributable to various components for this once-through plant with 0.50 \$/MMBtu gas. Capital servicing costs account for over one-third of the COE price. Even with 0.50 \$/MMBtu gas, the feedstock cost still constitutes about one-quarter of the total COE price. The $34 \%$ federal income tax rate constitutes about one-fifth of the total COE price.

\section{CONCLUSIONS AND RECOMMENDATIONS}

A conceptual plant design and cost estimates have been developed for a once-through. Fischer-Tropsch liquefaction plant with power co-production producing about 8,820 BPD of F-T liquids and 8+ MW of power from $100 \mathrm{MMSCF} /$ day of natural gas. The plant is estimated to cost about \$415 MM mid-1996 dollars. The economics are very sensitive to both the purchased gas price as well as the selling price of the co-produced power. With $0.50 \mathrm{~S} / \mathrm{MMBtu}$ gas and a reasonable electricity selling price of 3 cents per $\mathrm{kWh}$. the above plant will produce liquid transportation fuels which will be competitive with those produced from crude oil priced at about $18.8 \$ \mathrm{sbl}$.

Capital servicing costs still play a dominant role in driving the overall process economics. Optimization and site-specific studies are needed to explore various opportunities to simplify the overall process and reduce cost. Examples of such optimization include:

- The arbitrarily chosen design basis of $100 \mathrm{MMSCF} /$ day of gas feed should be adjusted for site-specific situations. Studies should be made to select an appropriate plant size which takes advantage of the economies of scale to optimize either the air separation and compression plant and/or the combined-cycle plant. the two most expensive sections of the plant.

- The use of sub-quality gas containing $\mathrm{CO}_{2}$ should be considered as a prime feedstock for this purpose (e.g., Alaska Prudhoe Bay associated gas has 10 to 15 mole $\% \mathrm{CO}_{2}$ ). The $\mathrm{CO}_{2}$ content of such gas might allow production of a syngas with a $\mathrm{H}_{2} / \mathrm{CO}$ ratio of 2.0 without requiring the separation and recycle of $\mathrm{CO}_{2}$ around the ATR.

Furthermore, this type of gas also might allow the use of an air-blown ATR which will eliminate the costly air separation plant at the expense of somewhat higher pressure slurry-bed F-T reactors and a more elaborate product recovery scheme to compensate for the higher inert concentration in the unconverted syngas.

- A minor simplification of the recovery scheme could be achieved if the plant were designed to produce a stabilized. pumpable syncrude instead of separate naphtha. distillate and butane streams. The latter are separated in this design mainly to provide a better basis for evaluating the value of the product.

- A small once-through, F-T plant might be ideal at a mine mouth location for processing coal-bed methane. The co-produced power could be used at the mine for processing the coal, and the hydrocarbon products could be shipped in the same manner as the coal, either by train or barge. Other examples of low-cost gas utilization can also be envisioned.

\section{REFERENCES:}

1. Topical Report VI, Vols. I and II. Natural Gas Fischer-Tropsch Case - Baseline Design/Economics for Advanced Fischer-Tropsch Technology, Contract No. DE-AC22-91PC90027, August. 1996.

2. Choi. G. N., Kramer. S. J., Tam, S. S. and J. M. Fox III. "Economics of a Natural Gas Based Fischer-Tropsch Plant." First Joint Power and Fuel systems Contractors' Conference. Pittsburgh. PA. July 9-11, 1996.

3. Topical Report I, Process Design - Illinois No. 6 Coal Case with Conventional Refining, Baseline Design/Economics for Advanced Fischer-Tropsch Technology, Contract No. DE-AC22-91PC90027, October, 1994.

4. Private communications, Lurgi Corporation - October 1996.

5. Final Report. Slurry Reactor Design Studies - Slurry vs. Fixed Bed Reactors for Fischer-Tropsch and Methanol, Contract No. DE-AC22-89PC89867, June. 1990.

6. Marano. J. J., Rogers. R. Choi. G. N., and Kramer, S. J., "Product Valuation of Fischer-Tropsch Derived Fuels", Symposium on Alternative Routes for the Production of Fuels. ACS National Meeting, Washington, D. C., August 21-26, 1994 
Table 1

Overall Plant Major Input and Output Flows

Feed

Natural Gas

Raw Water Make-up

Primary Products

F-T Naphtha

F-T Distillate

Butanes

Electric power
$100 \mathrm{MMSCF} /$ day

$5.3 \mathrm{MMgal} /$ day

$30.3 \mathrm{Mlbs} / \mathrm{hr}$

64.6 Mlbs/hr

$1.2 \mathrm{Mbb} / \mathrm{hr}$

2018 MW-hr/day
(17,800 MMBtu/hr)

(2,933 bbl/day)

(5736 bbVday)

(146 bbl/day)

(84.1 MW)

Table 2

Cost Breakdown of the Once-Through F-T Liquefaction Plant

$\begin{array}{cl}\text { Plant } & \text { Description } \\ 101 & \text { Air Compression \& Separation } \\ 102 & \text { Autothermal Reforming } \\ 103 & \mathrm{CO}_{2} \text { Removal and Recycle } \\ 201 & \text { Fischer-Tropsch Synthesis } \\ 202 & \text { Hydrogen Recovery } \\ 203 & \text { Product Fractionation } \\ 204 & \text { Wax Hydrocracking } \\ 31 & \text { Combined Cycle Plant } \\ & \text { Total ISBL } \\ & \text { Offsite } \\ & \end{array}$

HO Service/Fees \& Contingency

Total Cost:

\begin{tabular}{|c|c|}
\hline Cost (MMS) & $\%$ ISBL \\
\hline 70.4 & 32.7 \\
\hline 22.8 & 10.6 \\
\hline 13.4 & 6.2 \\
\hline 35.8 & 16.5 \\
\hline 3.6 & 1.7 \\
\hline 3.2 & 1.5 \\
\hline 11.8 & 5.5 \\
\hline 54.5 & 25.3 \\
\hline 215.5 & \\
\hline 120.3 & \\
\hline 335.8 & \\
\hline 79.4 & \\
\hline
\end{tabular}

415.2 
Figure 1

Once-Through Fischer-Tropsch (F-T) Design With Power Co-Production

(Overall Process Configuration)

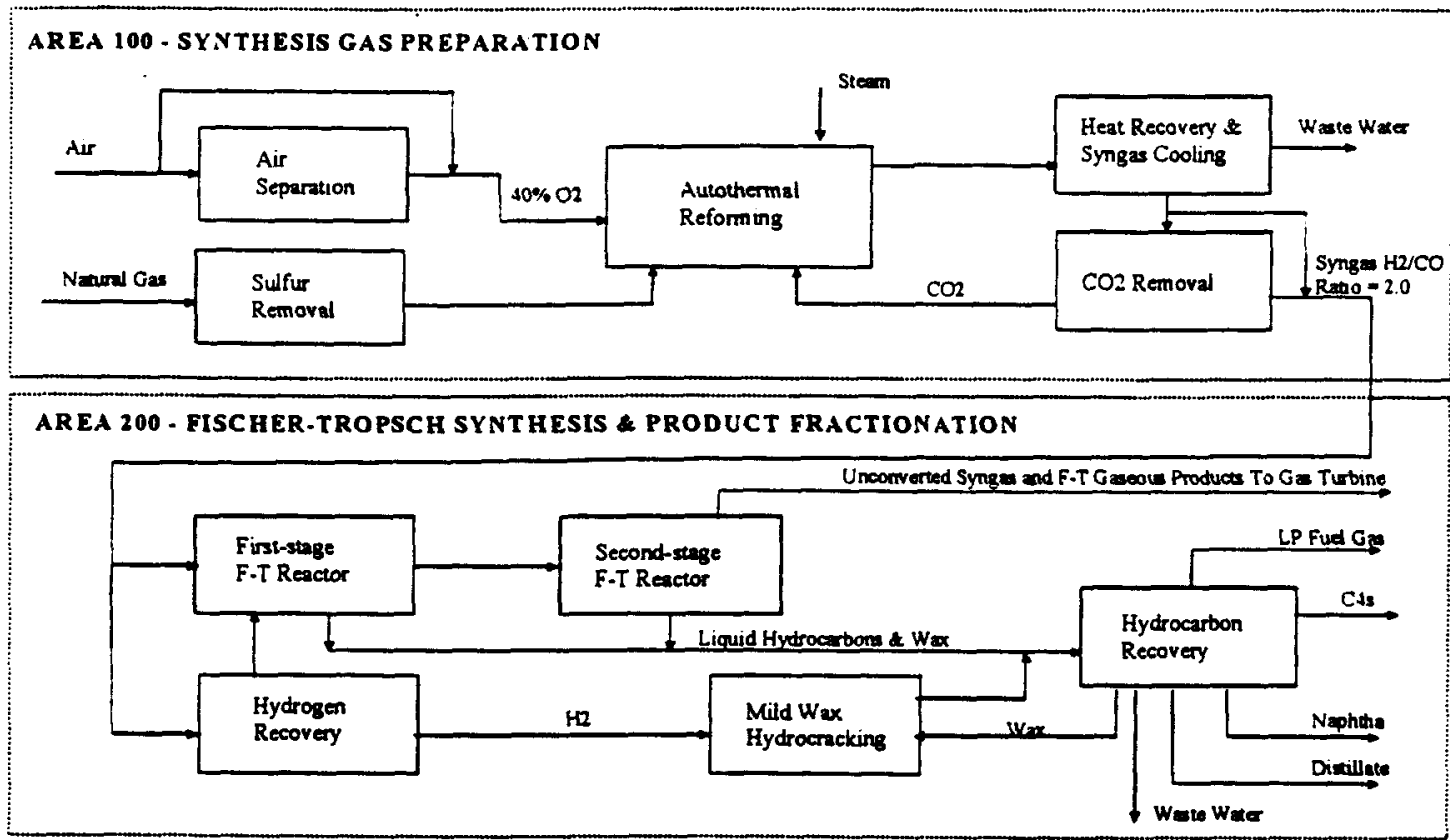

Figure 2

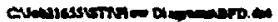

Block Flow Diagram - Combined Cycle Plant

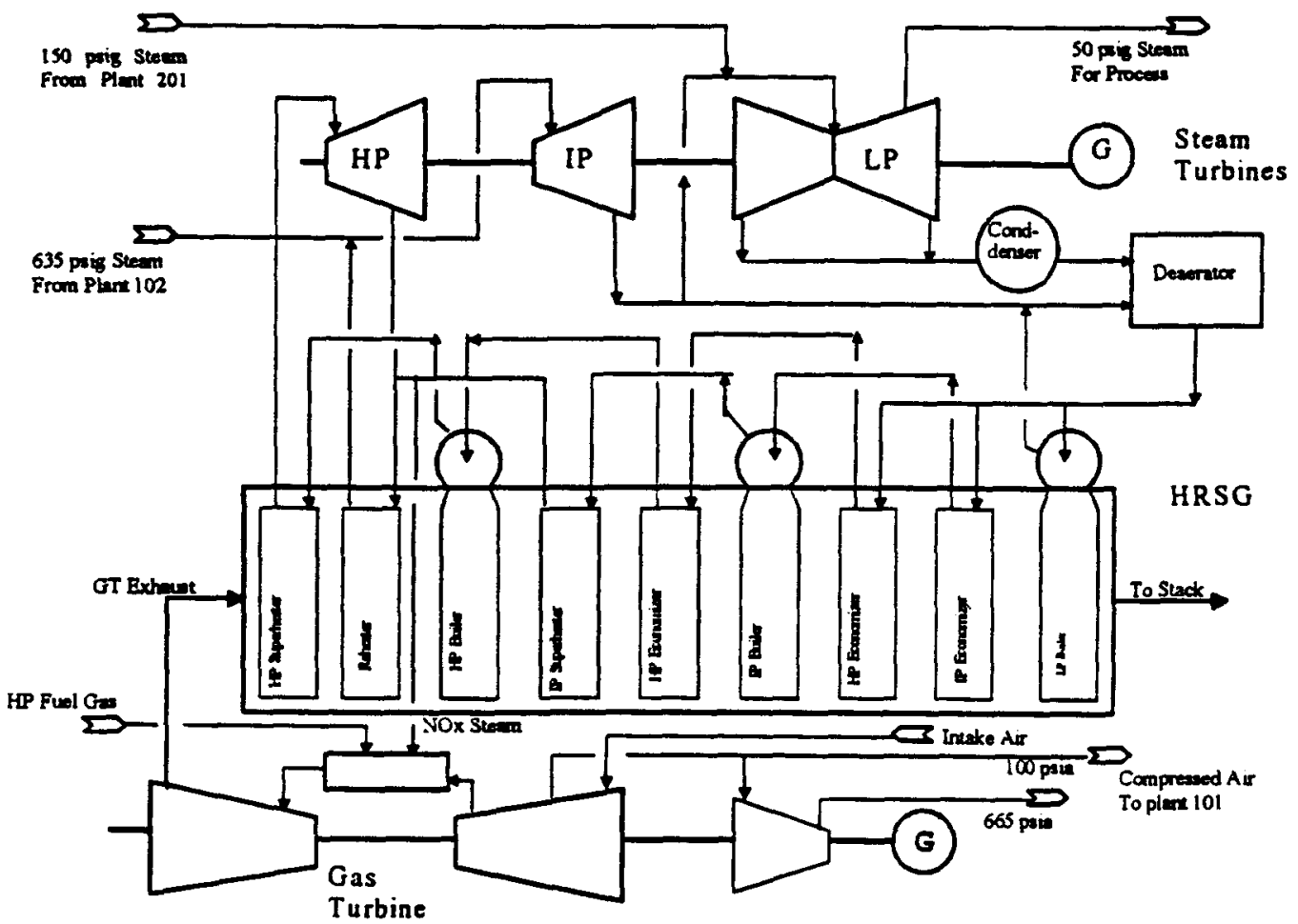


Figure 3

COE as a Function of Electricity Selling Price

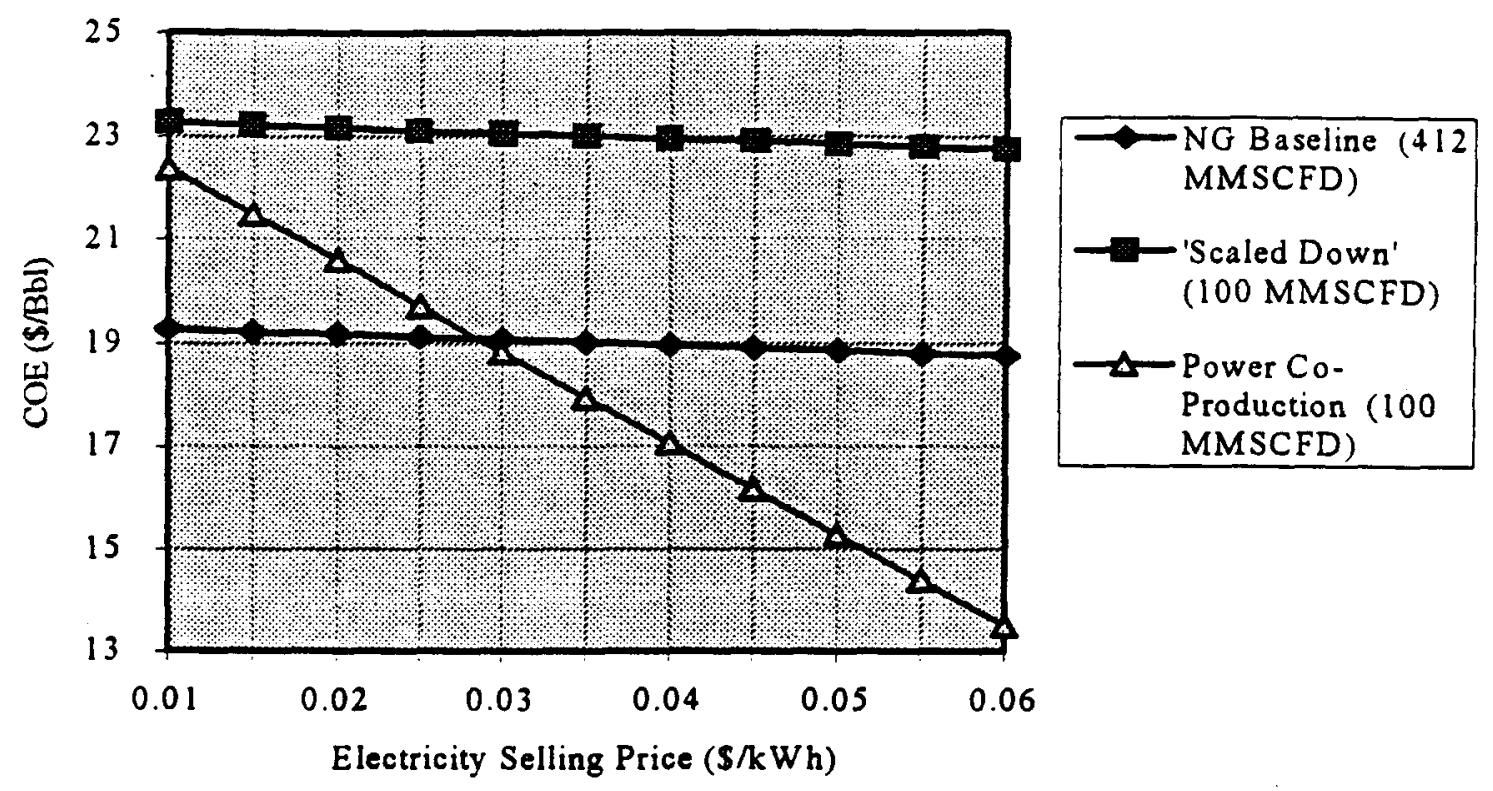

Figure 4

COE Cost Distribution With 0.5 \$MMMBtu Gas @ 0.03 \$/kWh Power Selling Price

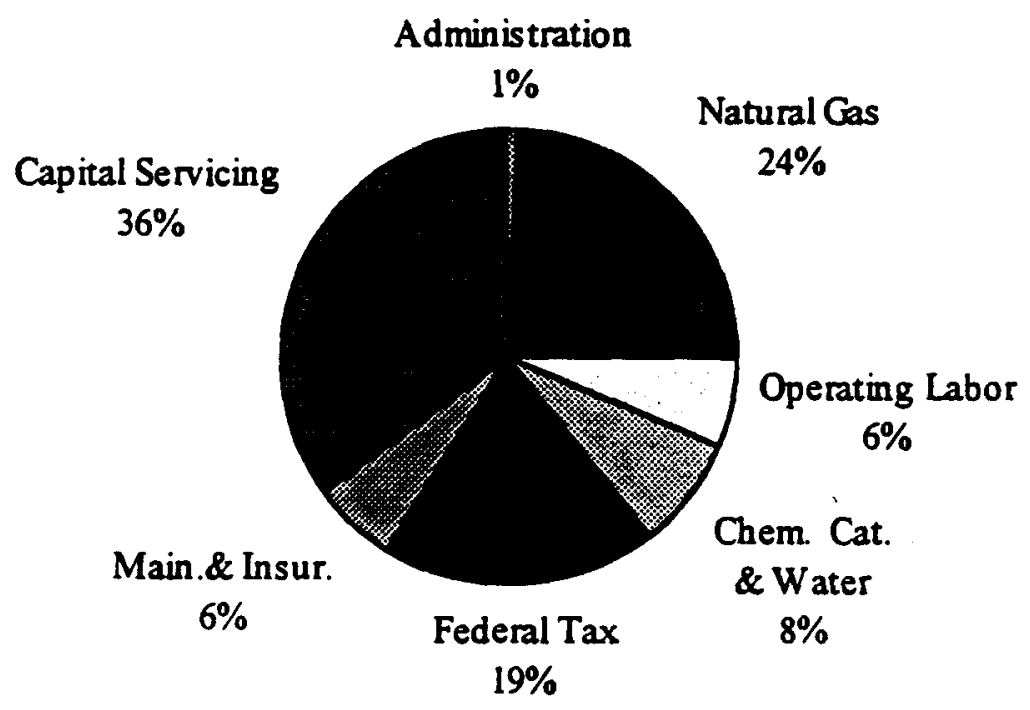

